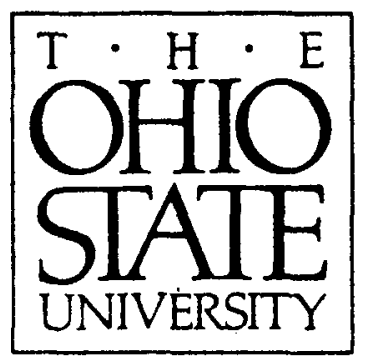

\title{
SCATTERING FROM THIN DIELECTRIC STRAPS SURROUNDING A PERFECTLY CONDUCTING STRUCTURE
}

\author{
Zeyad Al-Hekail \\ Inder J. Gupta

\section{The Ohio State University ElectroScience Laboratory} \\ Deportment of Eloctrical Engineering \\ Columbus, Ohio $\mathbf{4 3 2 1 2}$
}

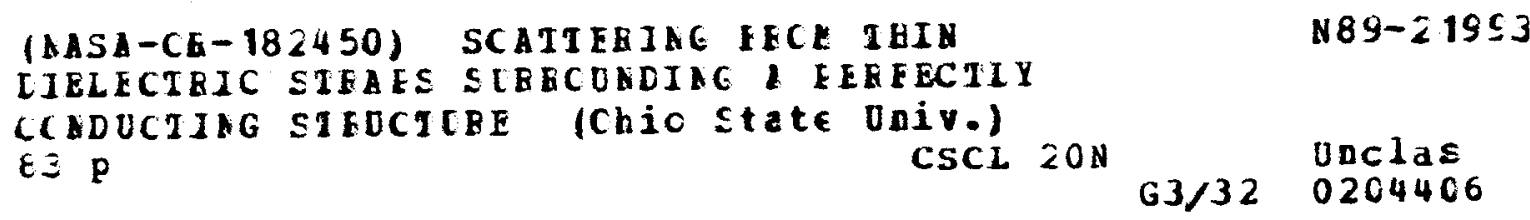

Technical Report 719493-5

Grant No. NSG 1613

January 1989

National Aeronautics and Space Administration

Langley Research Center

Hampton, VA 22217 


\section{NOTICES}

When Government drawings, specifications, or other data are used for any purpose other than in connection with a definitely related Government procurement operation, the United States Government thereby incurs no responsibility nor any obligation whatsoever, and the fact that the Government may have formulated, furnished, or in any way supplied the said drawings, specifications, or other data, is not to be regarded by implication or otherwise as in any manner licensing the holder or any other person or corporation, or conveying any rights or permission to manufacture, use, or sell any patented invention that may in any way be related thereto. 


\begin{tabular}{|c|c|c|}
\hline $\begin{array}{l}\text { REPORT DOCUMENTATION } \\
\text { PAGE }\end{array}$ & 1. AEPOAT NO. & 3. Rocipient's Accession No. \\
\hline \multirow{2}{*}{\multicolumn{2}{|c|}{$\begin{array}{l}\text { SCATTERING FROM THIN DIELECTRIC STRAPS SURROUNDING } \\
\text { A PERFECTLY CONDUCTING STRUCTURE }\end{array}$}} & $\begin{array}{l}\text { 5. Ropon Dote } \\
\text { January } 1989\end{array}$ \\
\hline & & a. \\
\hline \multirow{2}{*}{\multicolumn{2}{|c|}{$\begin{array}{l}\text { 7. Author(s) } \\
\text { Zeyad A1-Hekia1, Inder J. Gupta }\end{array}$}} & $\begin{array}{l}\text { a. Parformine Orcenization Rept. No. } \\
719493-5\end{array}$ \\
\hline & & 10. Project/Task/Work Unit No. \\
\hline \multicolumn{2}{|c|}{$\begin{array}{l}\text { The Ohio State University } \\
\text { ElectroScience Laboratory } \\
1320 \text { Kinnear Road } \\
\text { Columbus, Ohio } 43212\end{array}$} & $\begin{array}{l}\text { i1. Contract(C) or Grant(G) No. } \\
\text { (C) } \\
\text { (G) NSG } 1613\end{array}$ \\
\hline \multirow{2}{*}{\multicolumn{2}{|c|}{$\begin{array}{l}\text { 12. Sponsorine Orsenizotion Mome and Address } \\
\text { Nationa } 1 \text { Aeronautics and Space Administration } \\
\text { Langley Research Center } \\
\text { Hampton, VA } 22217\end{array}$}} & $\begin{array}{l}\text { 13. Type of Aopont a Period Covered } \\
\text { Technical Report }\end{array}$ \\
\hline & & 14. \\
\hline
\end{tabular}

15. Supplementery Notes

14. Abstract (Limit: 200 words)

A method to calculate the electromagnetic scattered fields from a dielectric strap wrapped around convex, conducting structure is presented. A moment method technique is used to find the current excited within the strap by the incident plane wave. Then, UTD is used to compute the fields scattered by the strap. Reasonable agreement was obtained between the computed and the measured results.

The results found in this study are useful in evaluating straps as a target support structure for scattering measurements.

17. Decument Anobyis o. Desertptors

b. Identiners/Open-Ended Terms

c. CosATI FNId/Group

12. Avelloblity exatement
19. Securty Closs (This Repont) Unclassified 20. Security Cless (This Peos) Unclassified 
TABLE OF CONTENTS

LIST OF FIGURES

I. INTRODUCTION

II. SCATTERING FROM THIN DIELECTRIC STRAPS SURROUNDING A PERFECTLY CONDUCTING CONVEX SURFACE

2.1 Introduction $\ldots \ldots \ldots \ldots \ldots \ldots \ldots \ldots \ldots \ldots \ldots$

2.2 The Current Induced in the Strap . . . . . . . . . . 8

2.3 Scattering from the Strap $\ldots \ldots \ldots \ldots \ldots$

2.3.1 Radiation From Sources Over Convex Surfaces . . . . 12

2.3.2 Stationary phase integration method . . . . . . 19

III. BACKSCATTERED FIELDS FROM A DIELECTRIC STRAP SURROUNDING A CONVEX STRUCTURE 21

3.1 Introduction $\ldots \ldots \ldots \ldots \ldots \ldots \ldots \ldots \ldots$

3.2 Description of the Ogive $\ldots \ldots \ldots \ldots \ldots \ldots$

3.3 Backscattering From the Strap $\ldots \ldots \ldots \ldots \ldots$

3.3.1 The Lit Part of the Strap . . . . . . . . . . . . 26

3.3.2 The Shadowed Part of the Strap . . . . . . . . 30 
IV. RESULTS AND EMPIRICAL DESIGN FORMULAS

4.1 Measured and computed results . . . . . . . . . . 37

4.2 Empirical Design Formulas . . . . . . . . . . . . 57

V. SUMMARY AND CONCLUSIONS 67

A. RAY DIVERGENCE FACTOR AND CAUSTIC DISTANCE 68

LIST OF REFERENCES

74 


\section{LIST OF FIGURES}

1 (a) Metal ogival pedestal. (b) Target on metal ogival pedestal . . . 2

2 (a)Styrofoam column. (b)Target on a styrofoam column. . . . . . 3

3 (a) A Dielectric strap. (b) Target attached to straps. (c)Target

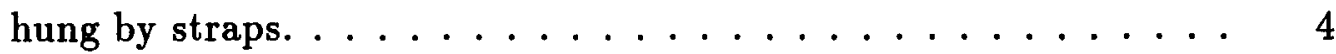

4 (a) The surface, $S$, surrounded by the strap. (b) The strap divided into sections. (c) Infinitely long strap existing over a ground plane tangent to the surface, $S$, at the section under consideration. . . 9

5 (a) An infinitely long strap over a ground plane. (b) The result of (a) after removing the ground plane and adding the image of the incident wave and the strap. (c) The strap width divided into cells. 11

6 Ray paths in the shadow and lit regions. . . . . . . . . 13

$\begin{array}{lll}7 & \text { Perspective view of a surface diffracted ray tube (enlarged view). . } & 17\end{array}$

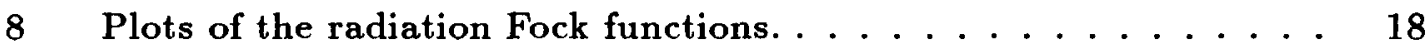

9 Principal surface directions at the source location. . . . . . . . 18

10 Structure surrounded by a dielectric strap. . . . . . . . . . 22

11 (a) An ogive described by $\phi$ and $Z$. (b) An ogive described by $\phi$

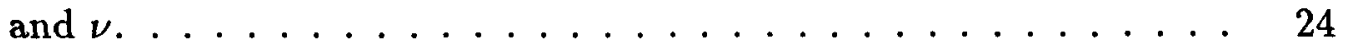

12 Direct radiation from a source mounted on an ogive. . . . . . . 27

13 Creeping wave radiation from a source mounted on an ogive. . . 32 
14 A dielectric strap wrapped around an ogive. . . . . . . . . .

15 Nose-on RCS of a strap versus frequency with 201 point smoothing. $\mathrm{a}=2.37^{\prime \prime}, \mathrm{b}=18.0^{\prime \prime}, \mathrm{L}=2^{\prime \prime}, \mathrm{T}=0.004^{\prime \prime}$ and $\epsilon_{r}=2.3 \ldots \ldots 39$

16 The measurement set up. .................. 40

17 Nose-on RCS of a strap versus frequency with 101 point smoothing. $\mathrm{a}=2.37^{\prime \prime}, \mathrm{b}=18.0^{\prime \prime}, \mathrm{L}=2^{\prime \prime}, \mathrm{T}=0.004^{\prime \prime}$ and $\epsilon_{r}=2.3$. . . . . . . .

18 Nose-on RCS of the tip of the ogive versus frequency with $a=2.37^{\prime \prime}$ and $b=18.0^{\prime \prime} \ldots \ldots \ldots \ldots \ldots \ldots$

19 RCS of a strap versus frequency with $a=2.37^{\prime \prime}, b=18.0^{\prime \prime}, L=2^{\prime \prime}$, $\mathrm{T}=0.004^{\prime \prime}$ and $\epsilon_{r}=2.3 \ldots \ldots \ldots \ldots 4 . \ldots \ldots$

20 RCS of a strap versus frequency with $a=2.37^{\prime \prime}, b=18.0^{\prime \prime}, L=2^{\prime \prime}$, $\mathrm{T}=0.004^{\prime \prime}$ and $\epsilon_{r}=2.3 \ldots \ldots \ldots \ldots \ldots$

21 RCS of a strap versus frequency with $a=2.37^{\prime \prime}, b=18.0^{\prime \prime}, L=2^{\prime \prime}$, $\mathrm{T}=0.004^{\prime \prime}$ and $\epsilon_{r}=2.3 \ldots \ldots \ldots \ldots \ldots$

22 RCS of a strap versus frequency with $a=2.37^{\prime \prime}, b=18.0^{\prime \prime}, L=2^{\prime \prime}$, $\mathrm{T}=0.004^{\prime \prime}$ and $\epsilon_{r}=2.3 \ldots \ldots \ldots \ldots \ldots$

23 RCS of a strap versus frequency with $a=2.37^{\prime \prime}, b=18.0^{\prime \prime}, L=2^{\prime \prime}$, $\mathrm{T}=0.004^{\prime \prime}$ and $\epsilon_{r}=2.3 \ldots \ldots \ldots \ldots$

24 RCS of a strap versus frequency with $a=2.37^{\prime \prime}, b=18.0^{\prime \prime}, L=2^{\prime \prime}$, $\mathrm{T}=0.004^{\prime \prime}$ and $\epsilon_{r}=2.3 \ldots \ldots \ldots \ldots$ 46

25 RCS of a strap versus frequency with $a=2.37^{\prime \prime}, b=18.0^{\prime \prime}, \mathrm{L}=2^{\prime \prime}$, $\mathrm{T}=0.004^{\prime \prime}$ and $\epsilon_{r}=2.3 \ldots \ldots \ldots \ldots \ldots \ldots$

26 RCS of a strap versus frequency with $a=2.37^{\prime \prime}, b=18.0^{\prime \prime}, L=2^{\prime \prime}$, $\mathrm{T}=0.004^{\prime \prime}$ and $\epsilon_{r}=2.3 \ldots \ldots \ldots \ldots$

27 Back scattered field from dielectric strap over a ground plane with $\mathrm{L}=2.5^{\prime \prime}, \mathrm{T}=0.004^{\prime \prime}, \epsilon_{r}=4.0$ and $\mathrm{f}=6 \mathrm{GHz} \ldots \ldots \ldots$ 


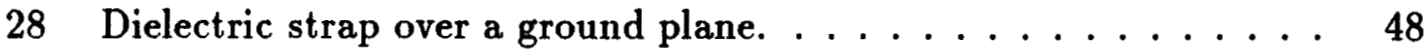

29 RCS of a strap wrapped around an ogive with $a=14.76^{\prime \prime}, b=29.53^{\prime \prime}$, $\mathrm{L}=3.69^{\prime \prime}$ and $\mathrm{f}=4 \mathrm{GHz} \ldots \ldots \ldots \ldots \ldots \ldots \ldots$

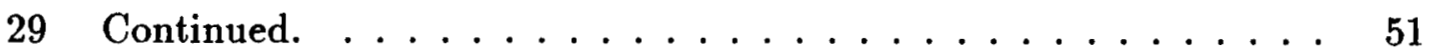

30 RCS of a strap wrapped around an ogive with $a=14.76^{\prime \prime}, b=29.53^{\prime \prime}$, $\mathrm{L}=2.36^{\prime \prime}$ and $\mathrm{f}=4 \mathrm{GHz} . \ldots \ldots \ldots \ldots \ldots \ldots \ldots \ldots$

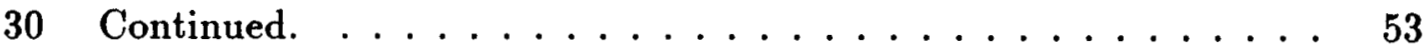

31 Nose-on RCS of the strap versus frequency with $a=14.76^{\prime \prime}, b=29.53^{\prime \prime}$ $, \mathrm{L}=2.36^{\prime \prime}, \mathrm{T}=0.004^{\prime \prime}$ and $\epsilon_{r}=4.0 \ldots \ldots \ldots \ldots \ldots$

32 RCS of a strap wrapped around an ogive with $a=2.37^{\prime \prime}, b=18^{\prime \prime}$, $\mathrm{L}=2.0^{\prime \prime}, \mathrm{T}=0.004^{\prime \prime}, \epsilon_{r}=2.3$ and $\mathrm{f}=4 \mathrm{GHz} \ldots \ldots \ldots \ldots \ldots$

33 RCS of a strap wrapped around an ogive with $a=2.37^{\prime \prime}, b=18^{\prime \prime}$, $\mathrm{L}=2.0^{\prime \prime}, \mathrm{T}=0.004^{\prime \prime}, \epsilon_{r}=2.3$ and $\mathrm{f}=10 \mathrm{GHz} . \ldots \ldots \ldots$

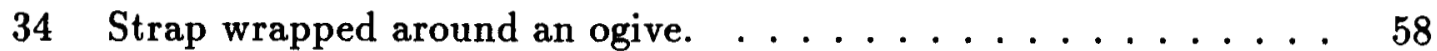

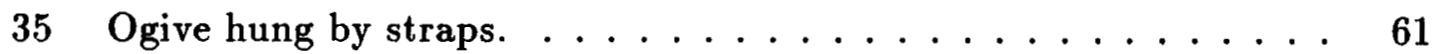

36 The angle $\theta_{s}$ with respect to the principle plane of the strap. . . 62

$37 \quad$ Nose-on RCS envelope of a strap wrapped around an ogive versus frequency with $a=2.37^{\prime \prime}, b=18^{\prime \prime}, \epsilon_{r}=2.3$ and $T=0.002^{\prime \prime}, 0.004^{\prime \prime}$ and $0.006^{\prime \prime} \ldots \ldots \ldots \ldots \ldots \ldots \ldots \ldots \ldots$

38 Nose-on RCS envelope of a strap wrapped around an ogive versus frequency with $a=2.37^{\prime \prime}, b=18^{\prime \prime}, T=0.004^{\prime \prime}$ and $\epsilon_{r}=2,3$ and 4.

39 RCS envelope of a strap wrapped around an ogive with $a=2.37^{\prime \prime}$, $b=18^{\prime \prime}, T=0.002^{\prime \prime}, 0.004^{\prime \prime}$ and $\epsilon_{r}=2,4$ and $\mathrm{f}=4 \mathrm{GHz} . \quad \ldots \ldots 64$

40 RCS envelope of the strap shown in Figure 35 versus frequency with $a=5^{\prime \prime}, b=20^{\prime \prime}, Z_{s}=10^{\prime \prime}, T=0.002^{\prime}, 0.004^{\prime \prime}$ and $\epsilon_{r}=2,4 . \ldots$ 
41 Creeping wave radiation from source over an ogive $\ldots \ldots 69$

42 Geodesic path on a developed cylinder. . . . . . . . . . . 70

43 Circular cylinder perturbation. .............. 71

44 The different parameters for the geodesic path. . . . . . . . . 72 


\section{CHAPTER I}

\section{INTRODUCTION}

During a radar cross section (RCS) measurement, the target is held in place using a support structure which can affect the accuracy of the measurement, especially when the target under test has a relatively low RC.S. Therefore, a support structure which has a low RCS is desired for scattering measurements. Among the commonly used structures are metal ogival pedestals, styrofoam columns and dielectric straps. These three structures are shown in Figures 1 through 3, respectively.

The metal ogival pedestal is commonly used as a target support because it can hold heavy targets and has a relatively low RCS, especially at high frequencies. Lai and Burnside $[1,2]$ have shown that the RCS of a metal ogival pedestal is directly proportional to the square of the wavelength of the incident field and is approximately $20 \mathrm{~dB}$ below a square meter at $1 \mathrm{GHZ}$. Thus, metal ogival pedestals do not appear to be a good choice for supporting targets at low frequencies; i.e. below $1 \mathrm{GHz}$. For a horizontally polarized incident wavefront the RCS of styrofoam columns is approximately equal to that of metal ogival pedestals while for vertical polarization the RCS of styrofoam columns is higher [2]. Furthermore, to support heavy targets the volume of the styrofoam columns should be rather large. Thus, styrofoam columns do not appear to be a good choice either. Gupta, Lai and Burnside $[2,3]$ studied the scattered fields from dielectric straps and concluded that 

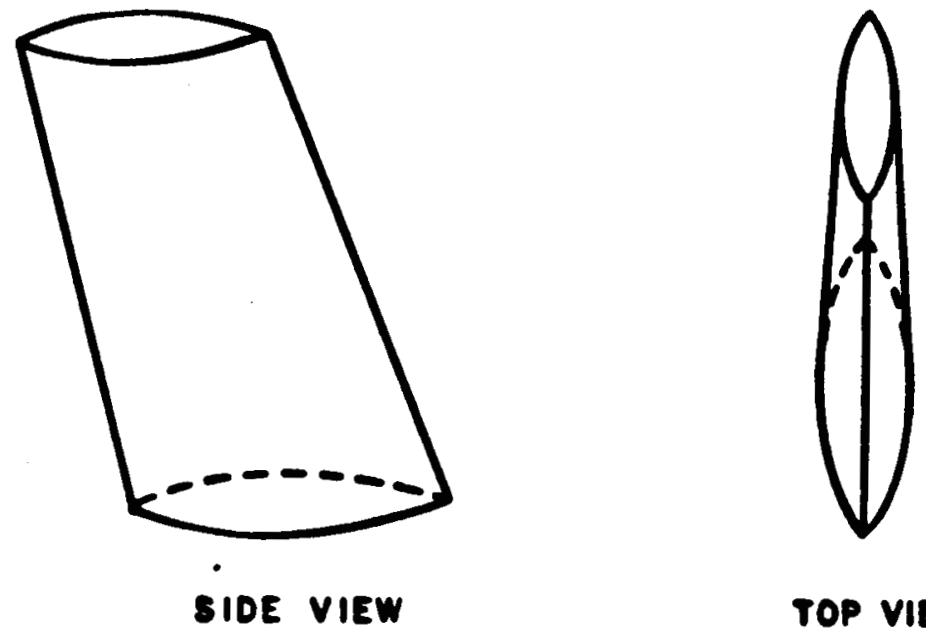

TOP VIEW

(a)

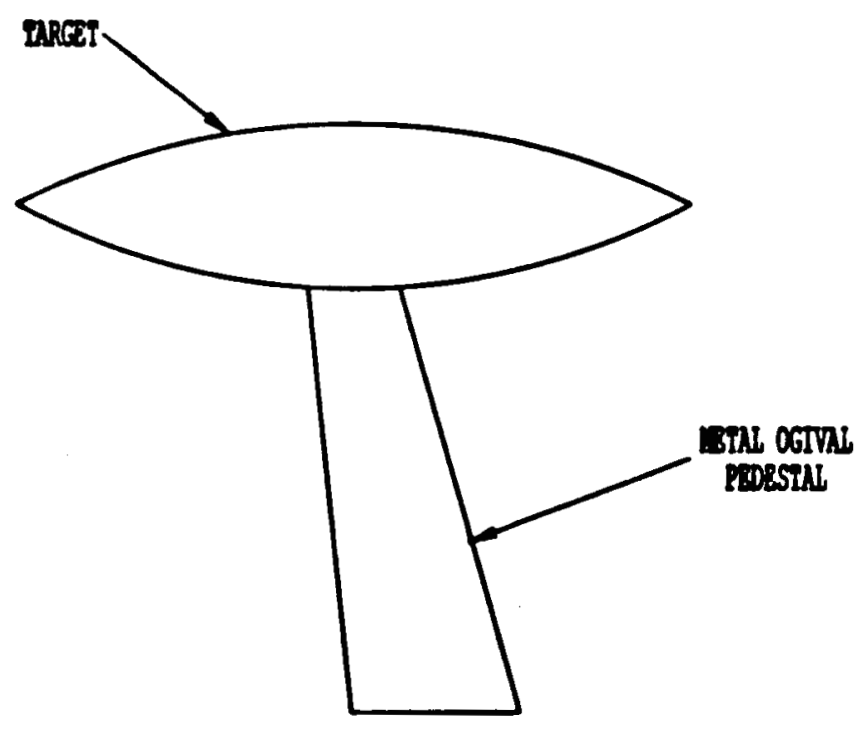

(b)

Figure 1: (a) Metal ogival pedestal. (b) Target on metal ogival pedestal 


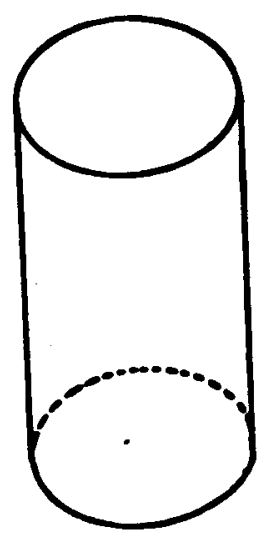

Vertical desion

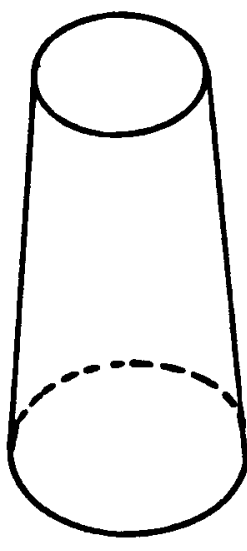

slented dealon

(a)

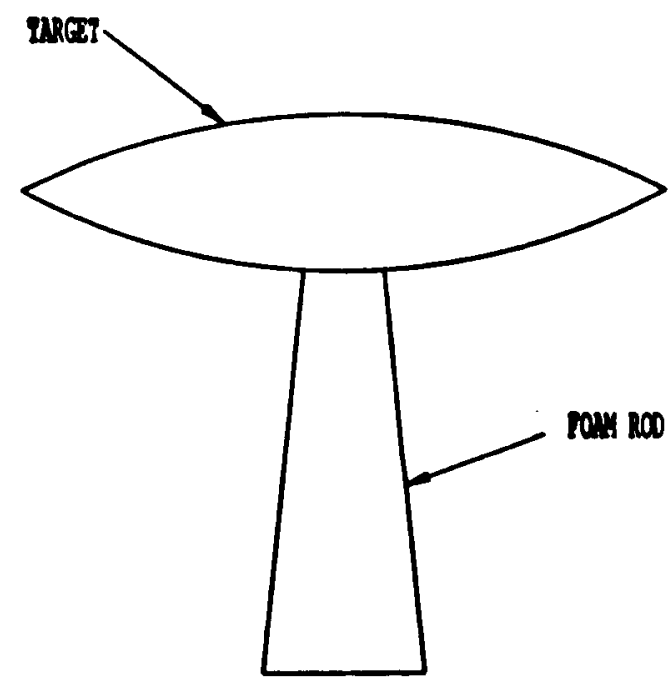

(b)

Figure 2: (a)Styrofoam column. (b)Target on a styrofoam column. 


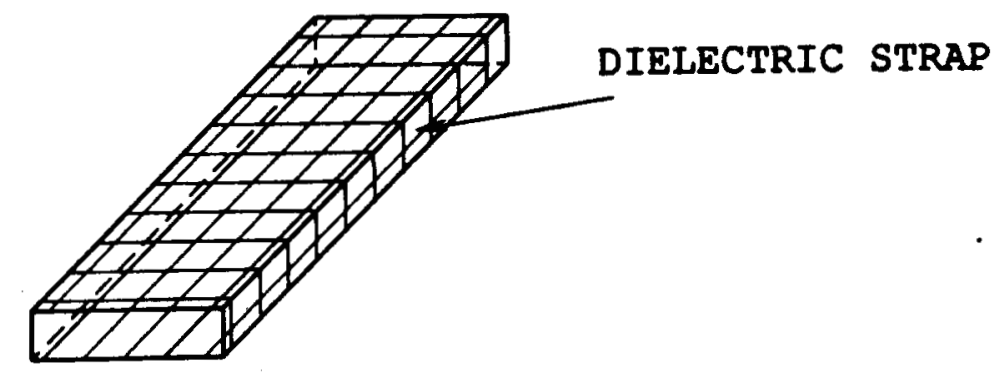

(a)

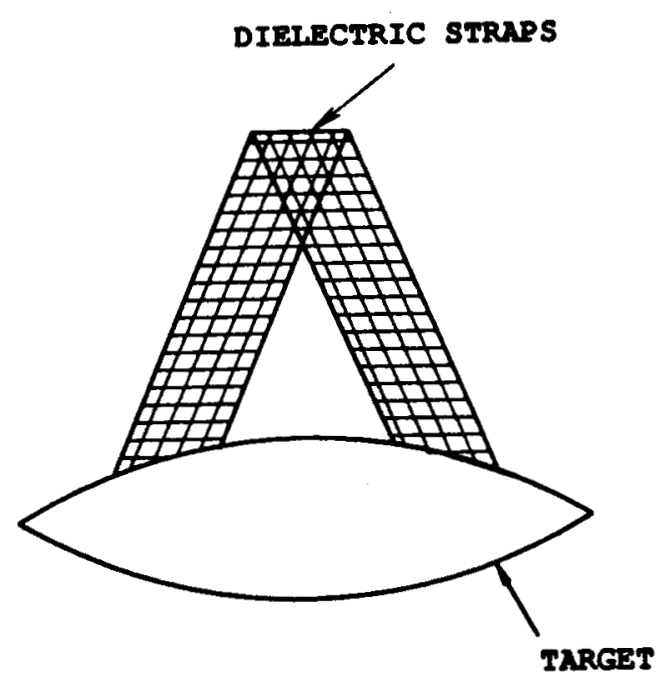

(b)

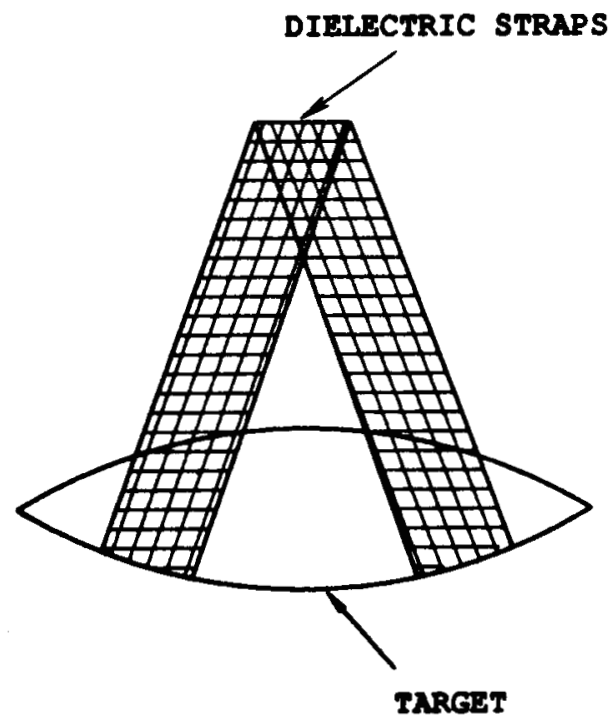

(c)

Figure 3: (a) A Dielectric strap. (b) Target attached to straps. (c)Target hung by straps. 
they perform much better at lower frequencies [2,3]. One advantage of the straps is that when the plane wave illuminates the straps at or near edge-on incidence, the RCS of the strap is quite low at low frequencies. Even though its RCS increases when the frequency increases, it is still lower than that for metal ogival pedestals. As a result,it is possible to align the straps with the low RCS direction of the target, so the minimum RCS of the target coincides with the minimum RCS of the strap. In addition, the straps can be built using composite material so they can handle very heavy targets. Therefore, the dielectric straps appear to be a good choice for supporting targets at low frequencies.

The straps can be used to hold a target using at least two basic methods. The first one is by connecting the straps directly to the target as shown in Figure $3(b)$. The second method is by wrapping the strap around the target as shown in Figure 3(c). The second method appears to be easier in that the target does not have to be modified. Both methods are expected to have a relatively low RCS when used as a target support. If the strap is directly attached to the target, the RCS of the support will be dependent on how well the strap is connected using a low scattering attachment. One the other hand, the second approach is dependent on the scattering level of the strap as it surrounds the structure. In order to evaluate the scattering level for this type of attachment, this report will determine the scattering performance of a thin dielectric strap surrounding a perfectly conducting structure.

The method of analysis can be outlined in the following steps. First, the current induced within the strap by an incident plane wave is found by employing the method of moments and the Uniform Geometrical Theory of Diffraction (UTD). After the current is found, the UTD is used in writing an integral for the radiated field based on the strap currents. The integral is simplified by using a station- 
ary phase point integration method. Then, the resulting expression is evaluated numerically. This method is used to evaluate the RCS of a strap surrounding a perfectly conducting structure, and reasonable agreement was found between the computed and the measured results.

The different chapters of this report are as follows. In Chapter II, a method is developed to compute the scattered fields from a thin dielectric strap surrounding a perfectly conducting convex surface. In Chapter III, the method is employed to solve for the backscattered field from a strap surrounding a perfectly conducting convex structure. Then, in Chapter IV some computed as well as measured results are presented. The conclusions are given in Chapter V. 


\section{CHAPTER II}

\section{SCATTERING FROM THIN DIELECTRIC STRAPS SURROUNDING A PERFECTLY CONDUCTING CONVEX SURFACE}

\subsection{Introduction}

In this chapter, a method is presented to find the electromagnetic fields scattered by a thin dielectric strap surrounding a perfectly conducting convex surface. First, a two-dimensional method is presented to determine the current excited across the width of the strap by an incident plane wave. This current is then extended to the three-dimensional case using high frequency techniques. Next, expressions for the fields radiated by a current element on a perfectly conducting convex surface are given using the Uniform Geometrical Theory of Diffraction (UTD). Finally, the total scattered field is written in the form of an integral of this current over the strap. The integration is then simplified by using a stationary phase technique.

Some of the assumptions which are used throughout this report are the following. The radius of curvature of the surface, $S$, is large compared to the wavelength of the incident wavefront, and the thickness, $T$, of the strap is much smaller than the wavelength. The dielectric material of the strap has a relative permeability, $\mu_{r}=1$, and an $\exp (j \omega t)$ time dependence is assumed and suppressed. Whenever the words "large" or "small" are used, they mean large or small relative to the 
wavelength.

\subsection{The Current Induced in the Strap}

One way to find the electromagnetic field scattered by a strap is to find the current induced within the strap by the incident electromagnetic wave. In this section, a method to find that current is described. In this study, a perfectly conducting surface, $S$, is surrounded by a strap of width, $L$, and thickness, $T$, as shown in Figure 4(a).

The first step in evaluating the current in the strap is to divide the strap into several smaller sections as shown in Figure 4(b). Then, the current inside each section is determined by assuming that the section under consideration is part of an infinitely long strap which exists over a ground plane tangent to the surface $S$ at the center of the section (see Figure 4(c)). One can see that three assumptions are used here. The first one is that each section can be treated alone, which implies that there are no interactions between the different sections. The second one is that each section exists over an equivalent ground plane. The last one is that the current inside a section can be found by considering that section to be part of an infinitely long strap. These three assumptions can be justified as follows.

The first assumption is based on the fact that, diffraction is a local phenomenon, which means that the scattering from any particular section is simply a function of the characteristics of that section only. This implies that the current in each section is a function of the characteristics of that section only. Hence, one can treat each section alone to find its associated current. The second assumption is made because the radius of curvature is large and the sections are small, such that each section appears to be mounted over a planar surface instead of a curved one. The third assumption is used because each section is part of a continuous strap. 

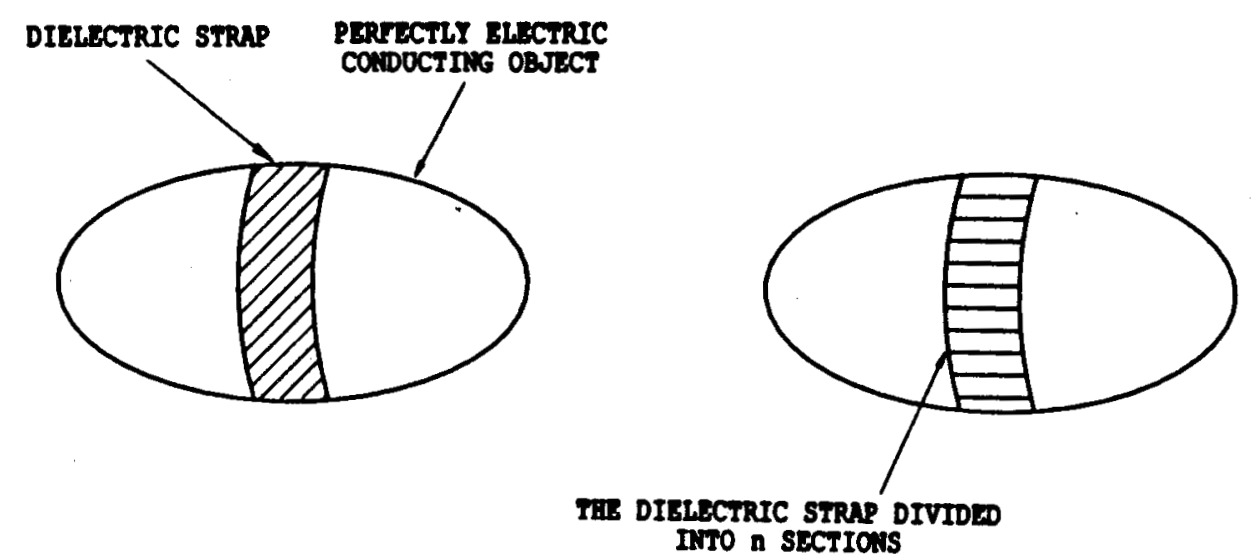

(a)

(b)

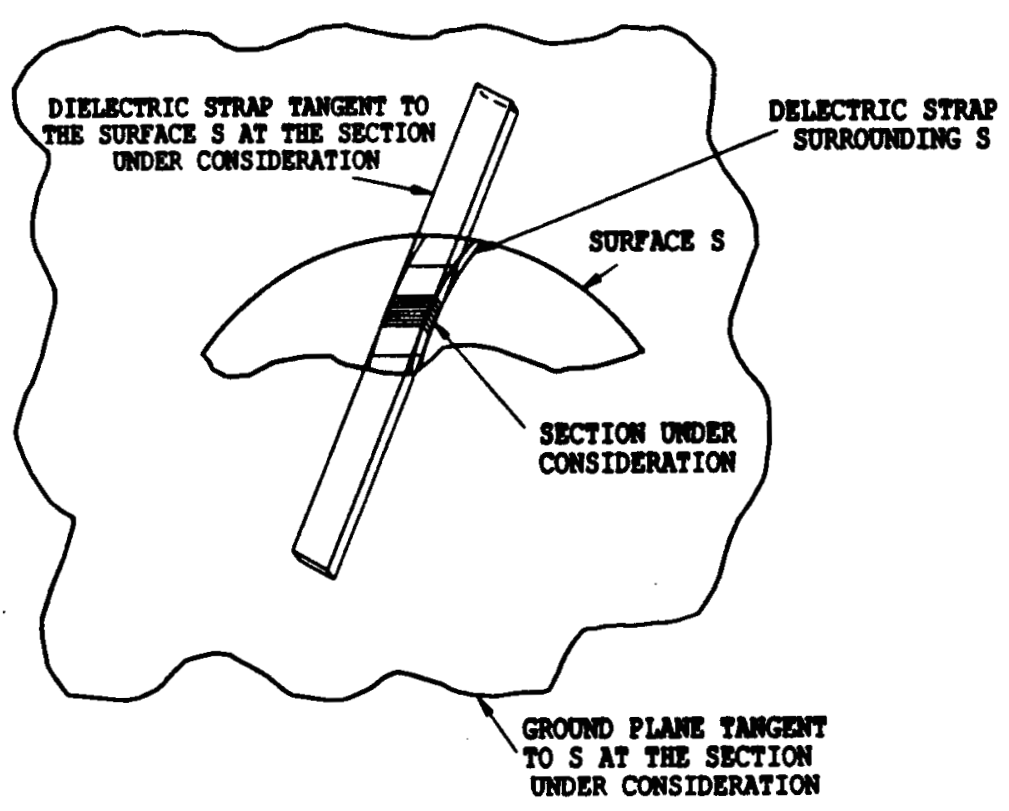

(c)

Figure 4: (a) The surface, $S$, surrounded by the strap. (b) The strap divided into sections. (c) Infinitely long strap existing over a ground plane tangent to the surface, $S$, at the section under consideration. 
So, the two terminations of each section do not contribute to the current. Therefore, the factors which affect the current in the section under consideration are the same as the factors which affect the current in an infinitely long strap. Based on the Geometrical Theory of Diffraction (GTD), these factors are the incident field, the diffraction from the two original edges forming the width of the strap and the interactions between these two edges. Therefore, the current in any particular section can be found by solving for the current in an infinitely long dielectric strap mounted over a ground plane which is tangent to the surface at the center of that section.

Now, let us find the current in the infinitely long strap by using the moment method technique. Consider an infinitely long strap mounted on a ground plane, see Figure 5(a), and excited by a plane wave which is given by

$$
\left.\bar{E}=e^{j k(X \cos \phi \sin \theta}+Y \sin \phi \sin \theta+Z \cos \theta\right) \hat{\phi}
$$

where $X, Y, Z, \theta$ and $\phi$ are shown in Figure 5 , and $k$ is the wavenumber of the incident field. The goal is to find the equivalent current inside the strap due to the incident plane wave. The first step is to get rid of the ground plane by adding the image of the structure. That is, double the thickness of the strap and add the image of the incident plane wave. By doing so, one obtains the problem shown in Figure 5(b). This problem can be solved by using the method of moments $[4,5]$. In the method of moments, the cross section of the dielectric strap is divided into cells which are small enough so that the electric field intensity is nearly uniform in each cell. The electric field intensities within each cell are initially considered to be an unknown quantities and a system of linear equations is generated by enforcing at the center of each cell that the total field must equal the sum of the incident 

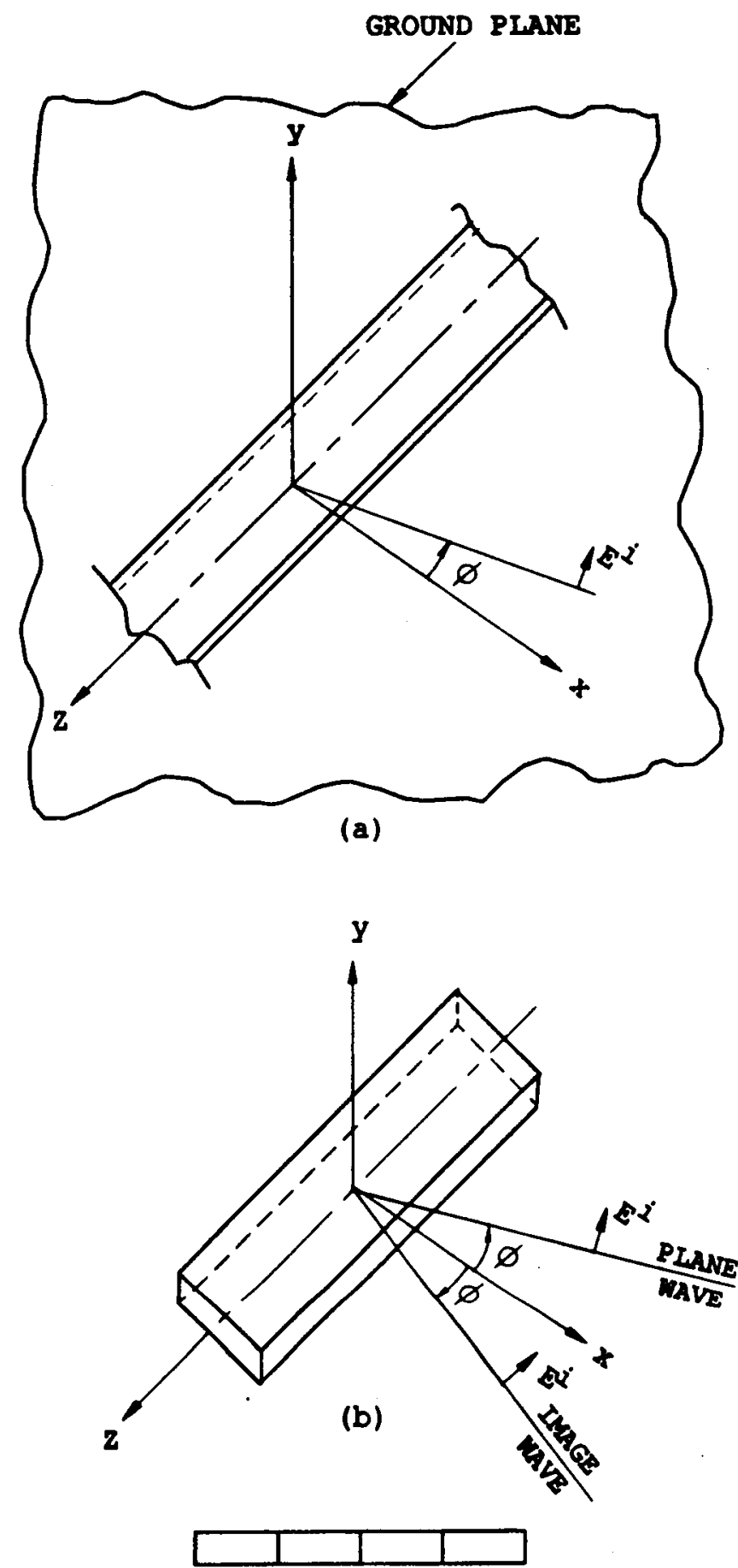

(c)

Figure 5: (a) An infinitely long strap over a ground plane. (b) The result of (a) after removing the ground plane and adding the image of the incident wave and the strap. (c) The strap width divided into cells. 
and scattered fields. This system of equations is then solved to obtain the electric field intensity in each cell. The equivalent electric current in each cell is then given by

$$
\bar{J}_{\text {cell } n}=j \omega\left(\epsilon-\epsilon_{o}\right) \bar{E}_{\text {cell } n}
$$

The above solution requires, as an input, the incident field at the center of each cell. For our particular problem the incident field at the strap mounted over the surface, $S$, shown in Figure 4 is different from the field incident at the strap mounted over an infinite ground plane as shown in Figure 5. This difference is related to the surface curvature at the location of the strap on the structure. This field is found by using high frequency techniques. First, the far field radiation by a source located on the surface, $S$, is found. Then, reciprocity is used to find the field on the surface, $S$, due to a source located in the far field. This field is used to find the current in the short dielectric strap section as described earlier.

\subsection{Scattering from the Strap}

At this point of our development the equivalent electric current in the strap is known. The radiated (scattered) electric field can be written using

$$
\bar{E}(P)=\iiint d \bar{E}\left(P / Q^{\prime}\right) d v^{\prime}
$$

where $d \bar{E}\left(P / Q^{\prime}\right)$ is the electric field at the observation point, $P$, due to an infinitesimal electric current moment at the source point, $Q^{\prime}$. By using UTD [6] one can find $d \bar{E}\left(P / Q^{\prime}\right)$. This is the topic of the next section.

\subsubsection{Radiation From Sources Over Convex Surfaces}

Consider an infinitesimal electric current moment, $d \bar{p}\left(Q^{\prime}\right)$, located on a perfectly electric conducting convex surface as shown in Figure 6. According to geo- 


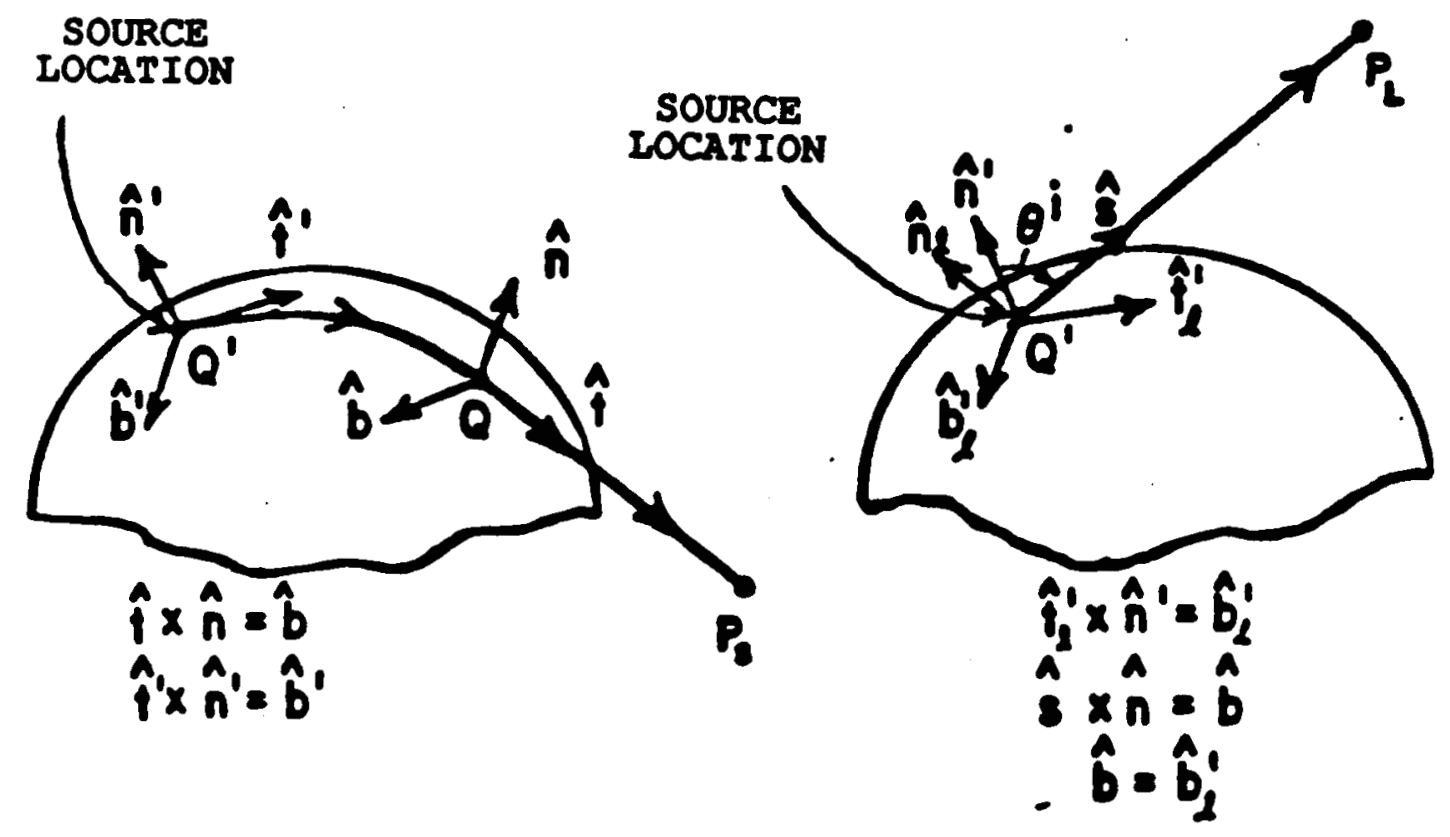

(a) shadow region

(b) lit region

Figure 6: Ray paths in the shadow and lit regions. 
metrical optics, the space surrounding the source is divided into an illuminated(lit) and shadow regions by a plane tangent to the surface at $Q^{\prime}$. This plane is referred to as the shadow boundary for that source location. The present formulation of the uniform GTD solution leads to separate representations for the elemental radiated field $d \bar{E}\left(P / Q^{\prime}\right)$ in the shadow and lit regions, respectively. However, these different representations have been shown to match exactly in polarization, amplitude and phase at the shadow boundary [7].

The unit vectors used in this treatment are shown in Figure 6. The unit vector, $\hat{s}$, defines the radiation direction. At the source point $\left(Q^{\prime}\right), \hat{n}^{\prime}$ is the unit surface normal vector, $\hat{t}^{\prime}$ is the unit tangent vector of the geodesic path, and the binormal unit vector is given by $\hat{b}^{\prime}=\hat{t}^{\prime} \times \hat{n}^{\prime}$. Similar unit vectors are introduced along the geodesic path. Thus at the diffraction point $(Q), \hat{n}$ is the unit surface normal vector, $\hat{t}$ is the unit tangent vector of the geodesic path and the binormal unit vector is given by $\hat{b}=\hat{t} \times \hat{n}$. Note that $\hat{n}_{L}$ is the unit vector normal to $\hat{s}$ and lies in the plane containing $\hat{n}^{\prime}$ and $\hat{s}$. Also, $\hat{t}_{L}^{\prime}$ is a unit tangent vector to the surface, $S$, at $Q^{\prime}$, and it lies in the plane containing $\hat{n}^{\prime}$ and $\hat{s}$. The binormal unit vector is defined by $\hat{b}_{L}^{\prime}$ which is given by $\hat{b}_{L}^{\prime}=\hat{t}_{L}^{\prime} \times \hat{n}^{\prime}$ or $\hat{b}_{L}^{\prime}=\hat{s} \times \hat{n}_{L}^{\prime}$.

In the lit region, one may express the elemental electric field produced by $d \bar{p}\left(Q^{\prime}\right)$ in a ray optical format as follows $[6]$ :

$$
d \bar{E}\left(P_{L}\right) \sim C d \bar{p}\left(Q^{\prime}\right) \quad \overline{\bar{T}}^{L} \frac{e^{-j k s}}{s}
$$

where $\frac{e^{-j k s}}{s}$ represents the spherical wave associated with the point source at $Q^{\prime}$. The quantity, $C$, is given by

$$
C=\frac{-j k}{4 \pi}
$$


Likewise, one may also express the field in the shadow region as follows [6]:

$$
d \bar{E}\left(P_{S}\right) \sim C d \bar{p}\left(Q^{\prime}\right) \cdot \overline{\bar{T}} \sqrt{\frac{d \psi_{o}}{d \eta(Q)}}\left\{\frac{\rho_{g}(Q)}{\rho_{g}\left(Q^{\prime}\right)}\right\}^{1 / 6} e^{-j k t} \sqrt{\frac{\rho_{2}^{d}}{s^{d}\left(\rho_{2}^{d}+s^{d}\right)}} e^{-j k s^{d}}
$$

The explicit forms of the radiation dyadic transfer functions, $\overline{\bar{T}}^{L}$ and $\overline{\bar{T}}$, are given by $[6]$

$$
\overline{\bar{T}}^{L}=Z_{o} \sin \theta^{i}\left\{\hat{n}^{\prime} \hat{n}_{L}\left(H^{L}+T_{o}^{2} F \cos \theta^{i}\right)+\hat{n}^{\prime} \hat{b} T_{o} F\right\}
$$

and

$$
\overline{\bar{T}}=Z_{o}\left\{\hat{n}^{\prime} \hat{n} H+\hat{n}^{\prime} \hat{b} T_{o} S\right\}
$$

where $t$ is the geodesic arc length from $Q^{\prime}$ to $Q$. The distance between the diffraction point, $Q$, and the observation point, $P$, is given by $s^{d}$ and $Z_{o}$ is the free space intrinsic impedance. The quantities $\left(H^{L}, H, S^{L}\right.$ and $\left.S\right)$ are referred as the hard $\left(H^{L} ; H\right)$ and soft $\left(S^{L} ; S\right)$ Fock functions which describe the radiation patterns of a source on a convex surface. These soft and hard Fock functions are plotted in Figure 8 and defined by [6]:

$$
\begin{gathered}
H^{L}\left(\xi^{L}\right)=\frac{e^{-j \xi^{L} / 3}}{\sqrt{\pi}} \int_{\infty e^{-j 2 \pi / 3}}^{\infty} d \tau \frac{e^{-j \xi^{L} \tau}}{w_{2}^{\prime}(\tau)} \\
H(\xi)=\frac{1}{\sqrt{\pi}} \int_{\infty e^{-j 2 \pi / 3}}^{\infty} d \tau \frac{e^{-j \xi \tau}}{w_{2}^{j}(\tau)} \\
S^{L}\left(\xi^{L}\right)=\frac{-j}{m\left(Q^{\prime}\right)} \cdot \frac{e^{-j \xi^{L} / 3}}{\sqrt{\pi}} \int_{\infty e^{-j 2 \pi / 3}}^{\infty} d \tau \frac{e^{-j \xi^{L} \tau}}{w_{2}(\tau)}, \text { and } \\
S(\xi)=\frac{-j}{m\left(Q^{\prime}\right)} \cdot \frac{1}{\sqrt{\pi}} \int_{\infty e^{-j 2 \pi / 3}}^{\infty} d \tau \frac{e^{-j \xi \tau}}{w_{2}(\tau)} .
\end{gathered}
$$


The Fock type Airy function is given by

$$
w_{2}(\tau)=\frac{1}{\sqrt{\pi}} \int_{\infty e^{-j 2 \pi / 3}}^{\infty} d \tau e^{(\tau t-t 3 / 3)}
$$

with $w_{2}^{\prime}(\tau)$ being the derivative of $w_{2}(\tau)$ with respect to $\tau$. The Fock parameter, $\xi$, for the shadow region is given by

$$
\xi=\int_{Q^{\prime}}^{Q} d t^{\prime} \frac{m\left(t^{\prime}\right)}{\rho_{g}\left(t^{\prime}\right)}
$$

with

$$
m\left(t^{\prime}\right)=\left[\frac{k \rho_{g}\left(t^{\prime}\right)}{2}\right]^{1 / 3}
$$

Here $\rho_{g}\left(t^{\prime}\right)$ is the surface radius of curvature along the ray path at $t^{\prime}$. The Fock parameter, $\xi^{L}$, for the lit region is given by

$$
\xi^{L}=-m^{L}\left(Q^{\prime}\right) \cos \theta^{i}
$$

with

$$
m^{L}\left(Q^{\prime}\right)=m\left(Q^{\prime}\right)\left[1+T_{o}^{2} \cos ^{2} \theta^{i}\right]^{-1 / 3}
$$

and $\cos \theta^{i}=\hat{n}^{\prime} \cdot \hat{s}$. The torsion factor, $T_{o}$, is given by

$$
T_{o}=T\left(Q^{\prime}\right) \rho_{g}\left(Q^{\prime}\right)
$$

where $T\left(Q^{\prime}\right)$ is the surface ray torsion at $Q^{\prime}$. One may show via differential geometry that [7]

$$
T\left(Q^{\prime}\right)=\frac{\sin 2 \alpha^{\prime}}{2}\left(\frac{1}{R_{2}\left(Q^{\prime}\right)}-\frac{1}{R_{1}\left(Q^{\prime}\right)}\right)
$$

and

$$
\frac{1}{\rho_{g}\left(Q^{\prime}\right)}=\frac{\cos ^{2} \alpha^{\prime}}{R_{1}\left(Q^{\prime}\right)}+\frac{\sin ^{2} \alpha^{\prime}}{R_{2}\left(Q^{\prime}\right)}
$$

where $R_{1}\left(Q^{\prime}\right)$ and $R_{2}\left(Q^{\prime}\right)$ denote the principal surface radii of curvature at $Q^{\prime}$, and $\alpha^{\prime}$ is the angle between $\hat{t}^{\prime}$ and $\hat{\tau}_{1}^{\prime}$ as shown in Figure 9 . Here $\hat{\tau}_{1}^{\prime}$ and $\hat{\tau}_{2}^{\prime}$ are the 


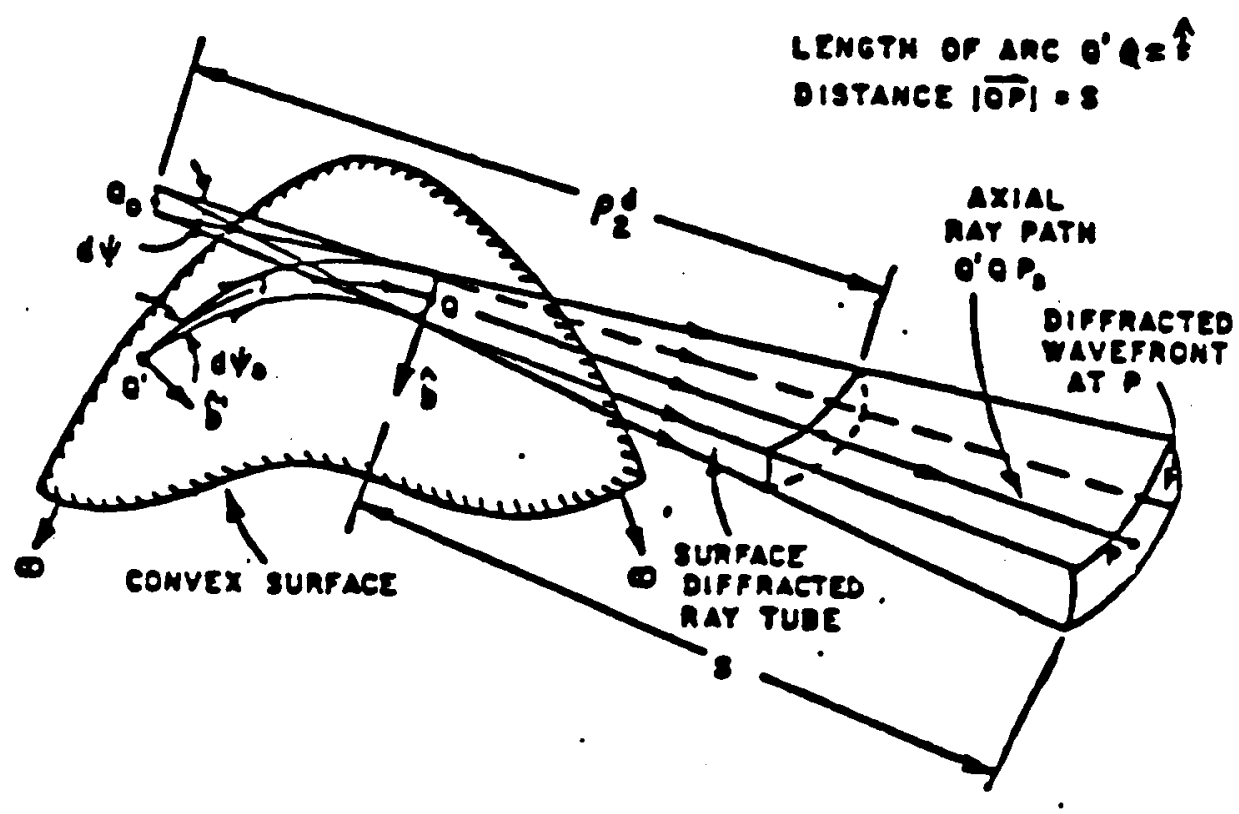

Figure 7: Perspective view of a surface diffracted ray tube (enlarged view).

principal surface directions at $Q^{\prime}$ associated with $R_{1}\left(Q^{\prime}\right)$ and $R_{2}\left(Q^{\prime}\right)$, respectively. The function, $F$, is given by

$$
F=\frac{S^{L}-H^{L} \cos \theta^{i}}{1+T_{o}^{2} \cos ^{2} \theta^{i}}
$$

The angles, $d \psi_{o}$ and $d \psi=\frac{d \eta(Q)}{\rho_{2}^{d}}$, are shown in Figure 7. Note that $\rho_{2}^{d}$ is the wave front radius of curvature, or the caustic distance, of the surface diffracted ray in the $\hat{b}$ direction at $Q$ and may be calculated via differential geometry.

Now, let us go back to Equation (2.2) and write it in the following form:

$$
\bar{E}(p)=\iint \bar{F}\left(\phi^{\prime}, t^{\prime}\right) e^{j \kappa \Phi\left(\phi^{\prime}\right)} e^{j t^{\prime} \beta} e^{-j k r_{o}} d \phi^{\prime} d t^{\prime}
$$

where

$t^{\prime}$ is the integration variable along the width of the strap 

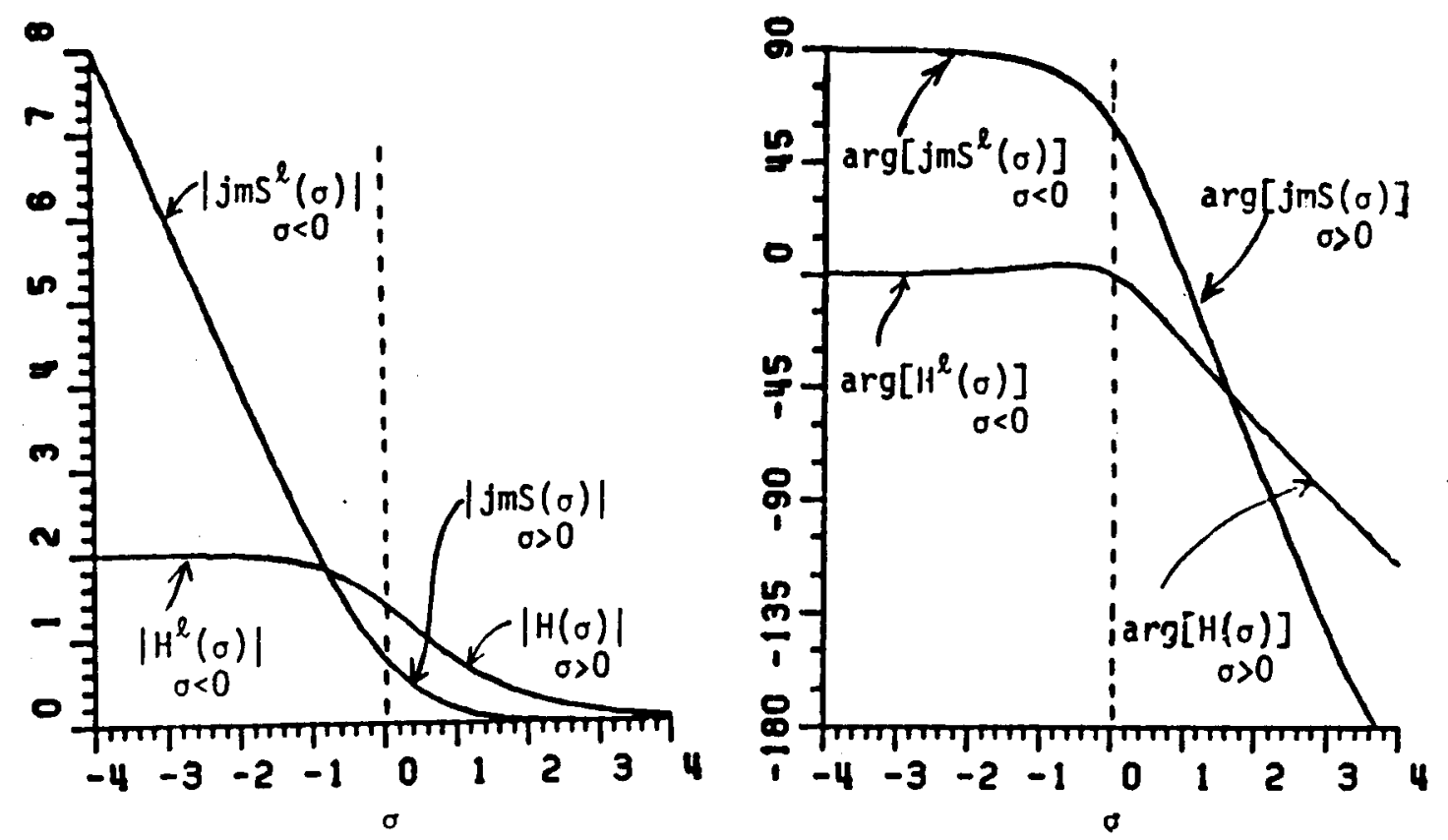

Figure 8: Plots of the radiation Fock functions.

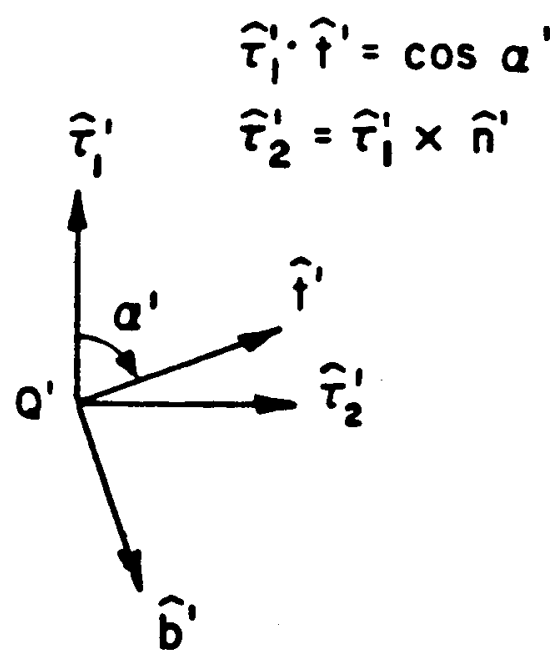

Figure 9: Principal surface directions at the source location. 
$\phi^{\prime}$ is the integration variable along the length of the strap

$e^{j \kappa \Phi\left(\phi^{\prime}\right)}$ contains the current and the radiation phase variation along the strap

$e^{j t^{\prime} \beta}$ contains the radiation phase variation along the width of the strap.

$\bar{F}\left(\phi^{\prime}, t^{\prime}\right)$ contains the remaining variables and constants. It does not contain any phase variation related to $\phi^{\prime}$, and

$r_{o}$ is the distance from the center of the structure to the observation point.

The triple integral in Equation (2.2) is converted into a double integral in Equation (2.21) because the strap is very thin compared to the wavelength such that the current is uniform across its thickness. The latter integral can then be evaluated numerically; however, the use of the stationary phase method makes it much easier to evaluate as described in the next section.

\subsubsection{Stationary phase integration method}

In this section, the stationary phase integration method [8] is summarized. Let us consider the following integral:

$$
I(\kappa)=\int_{a}^{b} F(\phi) e^{j \kappa \Phi(\phi)} d \phi
$$

where

$\phi$ is a real variable

$\kappa$ is real, positive and large

$\Phi(\phi)$ is a real, continuous function with continuous derivatives for $a \leq \phi \leq b$, and 
$F(\phi)$ may be a complex function, but it is slowly varying and well behaved in the range of integration.

Let $\Phi^{\prime}\left(\phi_{s}\right)=0$ for $a<\phi_{s}<b$, and $\phi_{s}$ is not close to $a$ and $b$. Then, $\phi_{s}$ is called the stationary phase point, and the integral, $I$, can be approximated by

$$
\begin{aligned}
I(\kappa) \sim & F\left(\phi_{s}\right) \sqrt{\frac{2 \pi}{\kappa\left|\Phi^{\prime \prime}\left(\phi_{s}\right)\right|}} e^{j\left[\kappa \Phi\left(\phi_{s}\right)+\frac{\pi}{4} \operatorname{sgn}\left(\Phi^{\prime \prime}\left(\phi_{s}\right)\right)\right]} \\
& +\frac{1}{\kappa} \frac{F(b)}{\Phi^{\prime}(b)} e^{j\left[\kappa \Phi(b)-\frac{\pi}{2}\right]}-\frac{1}{\kappa} \frac{F(a)}{\Phi^{\prime}(a)} e^{j\left[\kappa \Phi(a)-\frac{\pi}{2}\right]}
\end{aligned}
$$

where $\operatorname{sgn}(x)=-1$ if $x<0$, and $\operatorname{sgn}(x)=1$ if $x>0$. The above results were found by dividing the integral in Equation (2.22) into three integrals and then by using an asymptotic approximation to evaluate these integrals. The first term in Equation (2.23) corresponds to the contribution from the stationary point, $\phi_{s}$. The other two terms correspond to the contributions from the end points of the integration $a$ and $b$. If the function, $\Phi(\phi)$, has more than one stationary point in the interval $(a, b)$, one needs to sum the contributions from each of the stationary points. If the integration in Equation (2.22) is a closed loop integration then, there are no end point contributions.

In using the stationary phase integration method to simplify the integral in Equation (2.21) one needs to split the integral into two integrals. One of these two integrals covers the part of the strap which is excited directly by the incident wave. The other one covers the part of the strap which is excited by a creeping wave in that the strap is shadowed by the structure. Apparently, each integral contains one stationary point without an end point.

In the next chapter, a method to find the scattered electromagnetic field from a strap surrounding a perfectly conducting convex structure is developed based on the current found in this chapter. 


\section{CHAPTER III}

\section{BACKSCATTERED FIELDS FROM A DIELECTRIC STRAP SURROUNDING A CONVEX STRUCTURE}

\subsection{Introduction}

In this chapter, the method described in Chapter II is used to evaluate the backscattered fields from a thin dielectric strap wrapped around a structure such as shown in Figure 10. Recall that the relative permeability of the strap is assumed to be unity; while, the dielectric constant of the strap is $\epsilon_{r}$. For simplicity the pointing vector of the incident wavefront lies in a principal plane of the structure; i.e., the $X Z$ plane in Figure 10. The backscattered fields are computed in the Eplane ( $X Z$ plane in Figure 10). The H-plane backscattered fields from a thin strap wrapped around a structure are extremely low and one does not need to worry about their effect on the a measurement in that the worst case E-plane scattered fields set the measurement error level. This claim is justified at the end of this chapter.

The techniques developed here can be used to treat an arbitrary convex structure; however, the remaining discussion will be devoted to the ogive. The ogive is interesting in that it represents a low level scattering target; thus, one can observe the effect of the strap scattering for a practical case. This will become more apparent in the next chapter, when the results are examined.

In the case of a strap wrapped around an ogive there are two different mecha- 


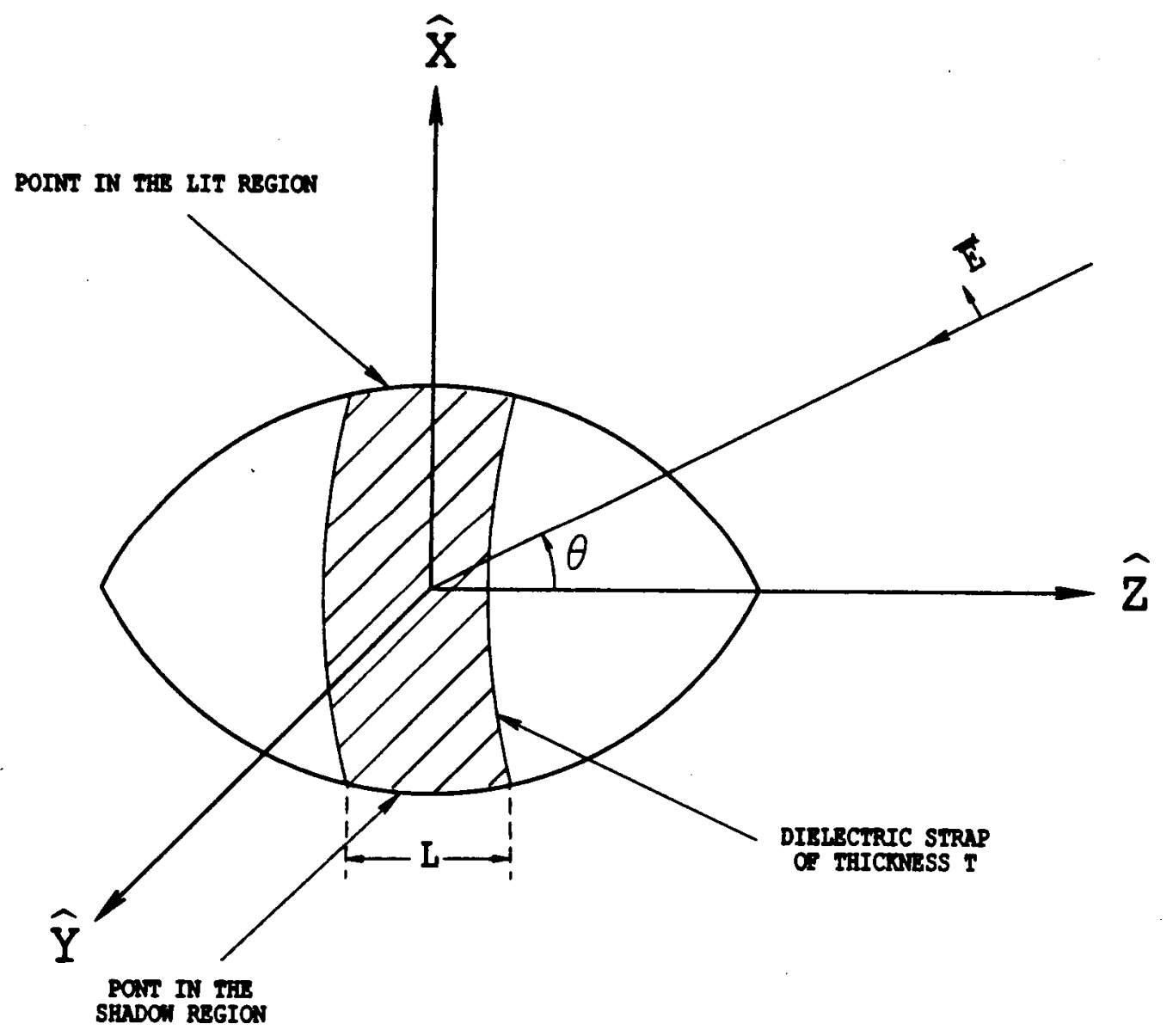

Figure 10: Structure surrounded by a dielectric strap. 
nisms leading to the currents excited on the strap and then to the scattered fields from the current elements. The first one is the direct excitation which occurs in the lit part of the strap. The second one is the creeping wave excitation which occurs in the shadowed part of the strap. These two mechanisms are treated separately.

\subsection{Description of the Ogive}

The ogive is used in this study to represent a general convex structure. It is shown in Figure 10 and can be described by knowing the two dimensions of the ogive, $a$ and $b$, as shown in Figure 11 . The vector $\bar{R}(\phi, Z)$ which connects the origin and a point on the surface of the ogive is then given by

$$
\bar{R}(\phi, Z)=A(Z) \cos (\phi) \hat{x}+A(Z) \sin (\phi) \hat{y}+Z \hat{z}
$$

where

$$
\begin{gathered}
A(Z)=-c+\sqrt{r^{2}-Z^{2}} \\
r=\frac{b^{2}+a^{2}}{2 a}, \text { and } \\
c=r-a .
\end{gathered}
$$

Another, convenient, way to describe the ogive is the following:

$$
\bar{R}(\phi, \nu)=(r \cos (\nu)-c) \cos (\phi) \hat{x}+(r \cos (\nu)-c) \sin (\phi) \hat{y}+r \sin (\nu) \hat{z}
$$

where $\nu$ is as shown in Figure 11, and from which one can see that

$$
\sin (\nu)=\frac{Z}{r}
$$

The advantage of the second definition is that the derivative of $\bar{R}$ with respect to $\nu$ is tangent to the surface of the ogive and perpendicular to $\hat{\phi}$. Note that $\hat{\phi}$ is also tangent to the surface of the ogive at any point. So, the two tangents perpendicular to each other at any point on the surface of the ogive can be easily 


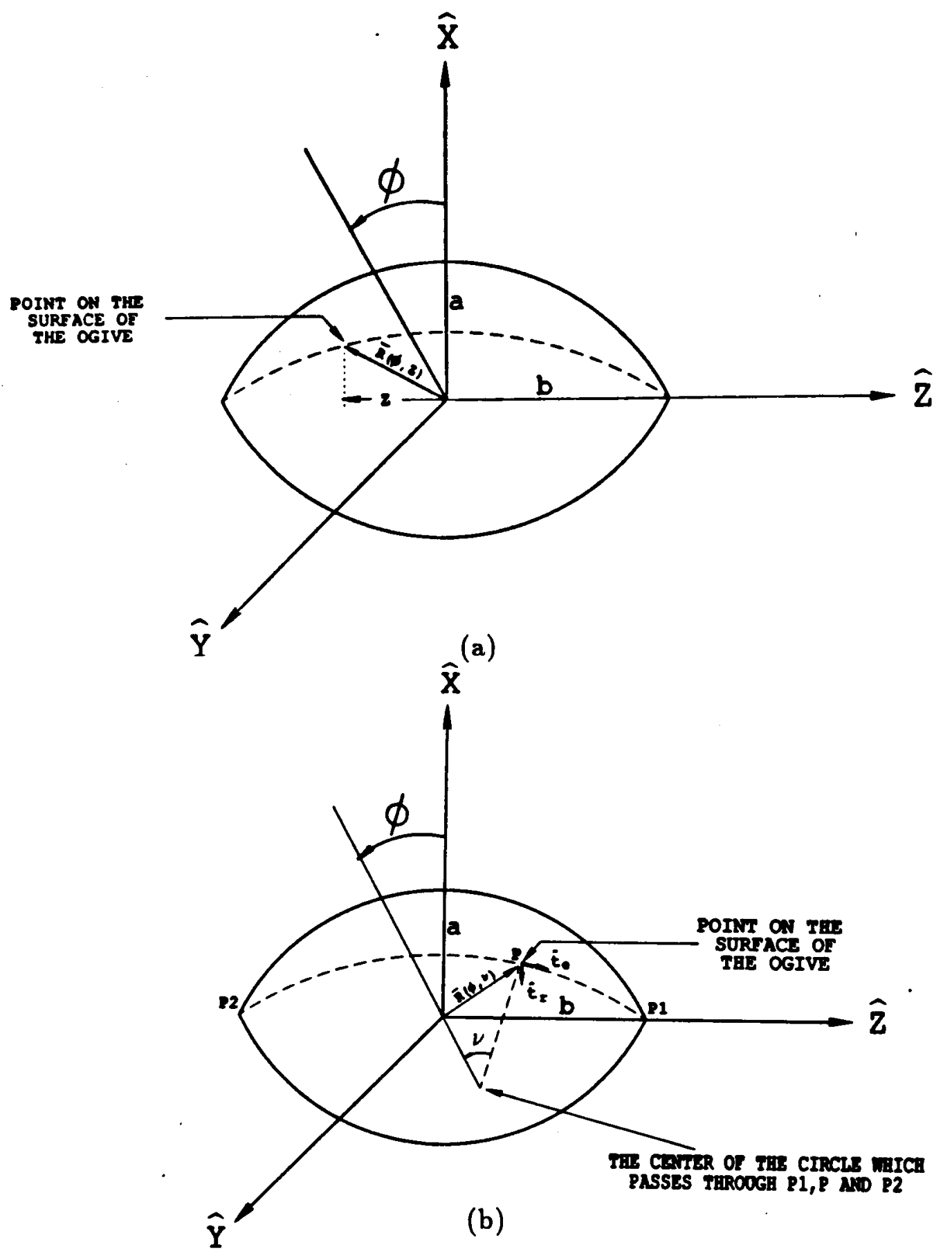

Figure 11: (a) An ogive described by $\phi$ and $Z$. (b) An ogive described by $\phi$ and $\nu$. 
found. These two tangents are enough to define the tangent to the surface at any direction. They are as follows:

$$
\begin{gathered}
\hat{t}_{r}=\frac{\bar{R}_{\phi}}{\left|\bar{R}_{\phi}\right|}=\hat{\phi}=-\sin (\phi) \hat{x}+\cos (\phi) \hat{y}, \text { and } \\
\hat{t}_{e}=\frac{\bar{R}_{\nu}}{\left|\bar{R}_{\nu}\right|}=-\sin (\nu) \cos (\phi) \hat{x}-\sin (\nu) \sin (\phi) \hat{y}+\cos (\nu) \hat{z}
\end{gathered}
$$

where

$$
\begin{gathered}
\bar{R}_{\phi}=\frac{\partial \bar{R}}{\partial \phi}, \text { and } \\
\bar{R}_{\nu}=\frac{\partial \bar{R}}{\partial \nu} .
\end{gathered}
$$

\subsection{Backscattering From the Strap}

Following the method described in Chapter II let us set up the integral for the radiation by the current induced in the strap as follows:

$$
\bar{E}(p)=\int_{0}^{2 \pi} \int_{-L / 2}^{L / 2} \bar{F}\left(\phi^{\prime}, t^{\prime}\right) e^{j \kappa \Phi\left(\phi^{\prime}\right)} e^{j t^{\prime} \beta_{e}} e^{-j k r_{o}} d \phi^{\prime} d t^{\prime} .
$$

The different constants and variables which appear in the above equation were defined earlier in Chapter II. The stationary phase integration method is used to evaluate the above integral with respect to $\phi^{\prime}$. The stationary points are located at the top and the bottom of the ogive; i.e. the points closest to and farthest from the transmitter/receiver antenna. The one at the bottom is shadowed by the ogive; while, the other one is not. This makes the integral formulation at each point different from the other one. Therefore, one must deal with each stationary point using a different method. 


\subsubsection{The Lit Part of the Strap}

In order to find the backscattered field from the strap, one needs to find the current within the strap excited by the incident plane wave. In order to find that current let us use the moment method approach described in Section 2.2. That method requires, as an input, the value of the incident field at the location of each cell in the strap. This can be done as follows. Consider a current moment located at the top of the ogive and radiating in free space as shown in Figure 12. The radiated field can be found using the formulas given in Section 2.3.1. So for an observation point located in the $X Z$ plane, the radiated field is given by

$$
d \bar{E} \sim \frac{-j k}{4 \pi} d \bar{p}(\bar{Q}) \cdot \overline{\bar{T}}^{L} \frac{e^{-j k s}}{s}
$$

where

$$
\begin{gathered}
\bar{T}^{L}=Z_{o} \sin \theta^{i} H^{L}\left(-m\left(Q^{\prime}\right) \cos \theta^{i}\right) \hat{n}^{\prime} \hat{n}_{L}, \text { and } \\
\bar{d} p\left(Q^{\prime}\right)=d p\left(Q^{\prime}\right) \hat{n}^{\prime}
\end{gathered}
$$

Then, one finds that

$$
d \bar{E} \sim \frac{-j k}{4 \pi} Z_{o} d p\left(Q^{\prime}\right) \sin \theta^{i} H^{L}\left(-m\left(Q^{\prime}\right) \cos \theta^{i}\right) \frac{e^{-j k s}}{s} \hat{n}_{L}
$$

The different variables in the above equation were defined in Chapter II. Now, by using reciprocity, one can find the field on the surface due to a plane wave incidence. By doing so, the electric field at the surface, $S$, is given by

$$
H^{L}\left(-m\left(Q^{\prime}\right) \cos \theta^{i}\right) \sin \theta^{i} e^{j k a \sin \theta \cos \phi^{\prime}} e^{j k(Z \cos \theta)}
$$

The above equation is used in the moment method approach to find the current in the strap. That current is then used to find the backscattered field from the lit part of the strap. 


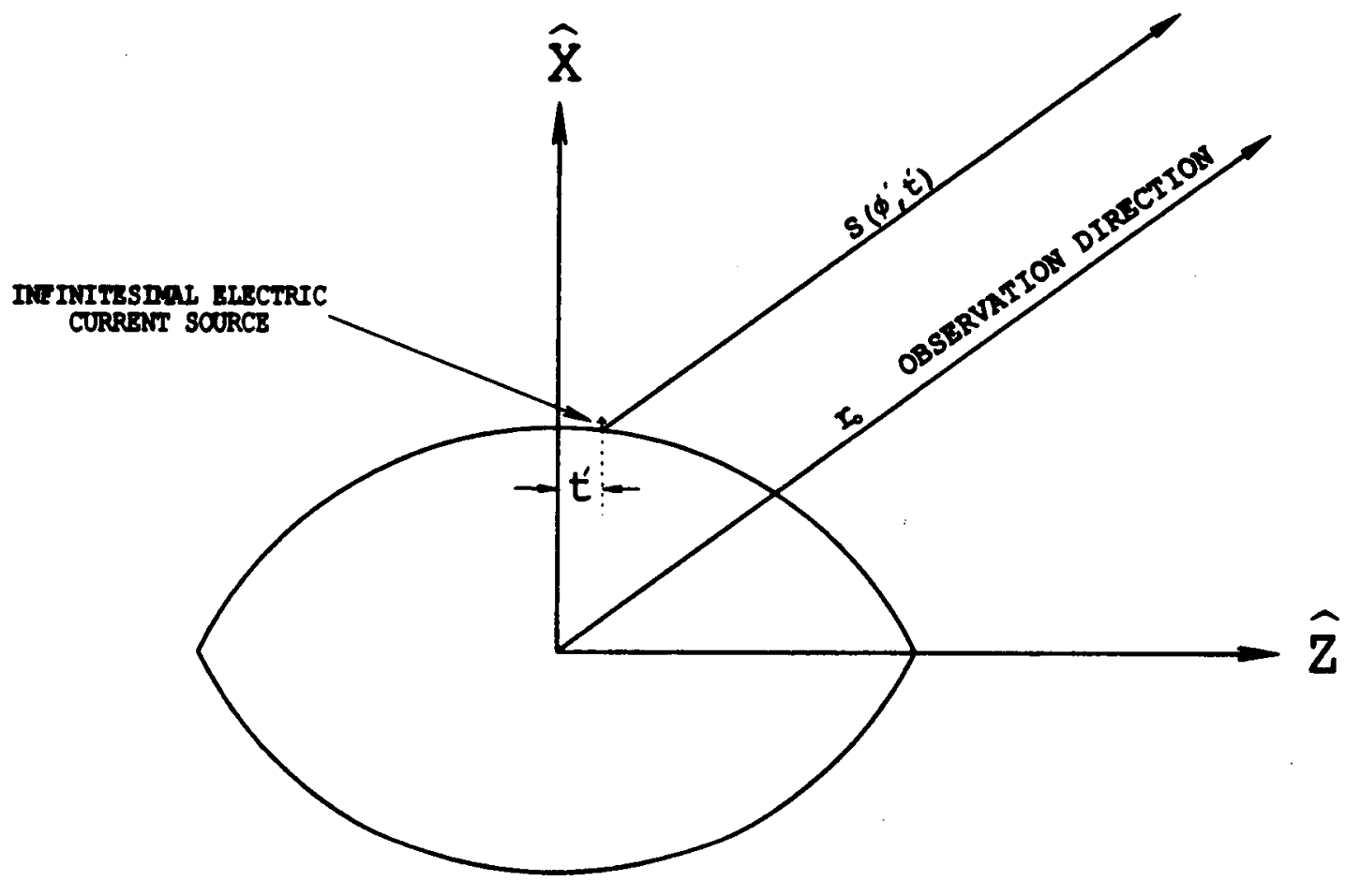

Figure 12: Direct radiation from a source mounted on an ogive. 
The radiated electric field by the current in the lit part of the strap is as follows:

$$
\bar{E}^{u}(p)=\frac{-j k T}{4 \pi} \int_{-L / 2}^{L / 2} \int_{-\phi_{b}}^{\phi_{b}} \bar{J}\left(\phi^{\prime}, t^{\prime}\right) \cdot \overline{\bar{T}} \frac{e^{-j k R}}{R} a d \phi^{\prime} d \hat{t}
$$

where

$Z_{o}$ is the intrinsic impedance of free space

$T$ is the thickness of the strap

$k$ is the wave number of free space

$R$ is the distance from the source point to the observation point

$\phi_{b}$ is the angle which defines the separation boundary between the shadowed and the lit part of the strap, and

$J\left(\phi^{\prime}, t^{\prime}\right)$ is the current in the strap.

Equation (3.14) can be written as

$$
\begin{gathered}
\bar{E}^{u}(P)=\frac{-j k}{4 \pi} Z_{o} T \frac{e^{-j k r_{o}}}{r_{o}} \int_{-L / 2}^{L / 2} \int_{-\phi_{b}}^{\phi_{b}} \sin \theta^{i} H^{L}\left(-m\left(Q^{\prime}\right) \cos \theta^{i}\right) \\
J\left(\phi^{\prime}, t^{\prime}\right) \hat{n}_{L} e^{j k\left(a \sin \theta \cos \phi^{\prime}\right)} e^{j k t^{\prime} \cos \theta_{a d \phi^{\prime}} d t^{\prime}}
\end{gathered}
$$

where

$\theta$ is the observation angle measured from the $Z$-axis, and

$r_{o}$ is the distance from the ogive to the observation point.

Now, the current can be described by

$$
\bar{J}\left(\phi^{\prime}, t^{\prime}\right)=\bar{J}_{1}\left(\phi^{\prime}, t^{\prime}\right) e^{j k a \sin \theta \cos \phi^{\prime}}
$$


Then, Equation (3.15) can be written in the following form:

$$
\bar{E}^{u}(p)=\int_{-L / 2}^{L / 2} \int_{-\phi_{b}}^{\phi_{b}} \bar{F}\left(\phi^{\prime}, t^{\prime}\right) e^{j \kappa \Phi\left(\phi^{\prime}\right)} e^{j t^{\prime} \beta} e^{-j k r_{o}} d \phi^{\prime} d t^{\prime}
$$

with

$$
\begin{gathered}
\bar{F}\left(\phi^{\prime}, t^{\prime}\right)=\frac{-j k}{4 \pi r_{o}} Z_{o} \sin \theta^{i} H^{L}\left(-m\left(Q^{\prime}\right) \cos \theta^{i}\right) T a J_{1}\left(\phi^{\prime}, t^{\prime}\right) \hat{n}_{L} \\
\kappa=2 k a \sin \theta \\
\Phi\left(\phi^{\prime}\right)=\cos \phi^{\prime}, \text { and } \\
\beta=k \cos \theta .
\end{gathered}
$$

Now, the above integral can be evaluated using the stationary phase integration method. First, the stationary phase point is found from

$$
\begin{gathered}
\phi\left(\phi^{\prime}\right)=\cos \phi^{\prime} \\
\Phi^{\prime}\left(\phi^{\prime}\right)=-\sin \phi^{\prime}, \text { and } \\
\Phi^{\prime \prime}\left(\phi^{\prime}\right)=-\cos \phi^{\prime}
\end{gathered}
$$

where the stationary point is given by

$$
\Phi^{\prime}\left(\phi_{s t}\right)=0 .
$$


In this case, the stationary points are given by $\phi_{s t}=0, \pi$. Since the interest here is in the lit part of the strap, $\phi_{s t}=0$ is the only stationary point to be evaluated here. Using the results of section 2.3.2, one finds that

$$
\bar{E}^{u}(P)=\int_{-L / 2}^{L / 2} \bar{F}\left(0, t^{\prime}\right) \sqrt{\frac{2 \pi}{\kappa\left|\Phi^{\prime \prime}\left(\phi_{s t}\right)\right|}} e^{j\left(\kappa \Phi\left(\phi_{s t}\right)+\frac{\pi}{4} \operatorname{sgn}\left(\Phi^{\prime \prime}\left(\phi_{s t}\right)\right)\right.} e^{j t^{\prime} \beta_{e} e^{-j k r_{o}} d t^{\prime}}
$$

which can be written as

$$
\bar{E}^{u}(p)=\sqrt{\frac{-j \pi}{k a \sin \theta}} e^{j 2 k a \sin \theta} e^{-j k r_{0}} \int_{-L / 2}^{L / 2} \bar{F}\left(0, t^{\prime}\right) e^{j t^{\prime} \beta} d t^{\prime}
$$

The above integral can then be easily evaluated numerically across the width of the strap.

\subsubsection{The Shadowed Part of the Strap}

According to Equation (2.5) the radiation by a current element in the shadowed part of the strap can be written as

$$
\begin{gathered}
\bar{E}^{s}(P)=\frac{-j k T}{4 \pi} \int_{-L / 2}^{L / 2} \int_{\phi_{b}}^{2 \pi-\phi_{b}} \bar{J}\left(\phi^{\prime}, t^{\prime}\right) \\
\cdot \overline{\bar{T}} \sqrt{\frac{d \psi_{o}}{\rho_{2}^{d} d \psi}}\left[\frac{\rho_{g}(Q)}{\rho_{g}\left(Q^{\prime}\right)}\right]^{1 / 6} e^{-j k t} \sqrt{\frac{\rho_{2}^{d}}{s^{d}\left(\rho_{2}^{d}+s^{d}\right)}} e^{-j k s^{d}} a d \phi^{\prime} d t^{\prime}
\end{gathered}
$$

where

$\bar{J}\left(\phi^{\prime}, t^{\prime}\right)$ is the current in the strap. This current is found in a similar way to that used for the current in the lit part of the strap. The details are given at the end of this section. The other factors in Equation (3.22) are defined as follows:

$\overline{\bar{T}}=Z_{o}\left[\hat{n}^{\prime} \hat{n} H(\xi)+\hat{n}^{\prime} \hat{b} T_{o} S(\xi)\right]$, but because of the symmetry the $\hat{b}$ component cancels out; therefore, one needs to consider

$$
\overline{\bar{T}}=Z_{o} \hat{n}^{\prime} \hat{n} H(\xi)
$$


$\sqrt{\frac{d \psi_{o}}{d \psi}}$ and $\rho_{2}^{d}$ are the ray divergence factor and the caustic distance, respectively. They are found using the cylinder perturbation method [9]. See Appendix A for details.

$\rho_{g}(Q)$ and $\rho_{g}\left(Q^{\prime}\right)$ are the surface radii of curvature in the $\hat{t}$ and $\hat{t}^{\prime}$ direction, respectively. They are given by Equation (2.17).

$t$ is the geodesic path length, which is given by

$$
t=-r \theta \cos \phi^{\prime}-t^{\prime}
$$

$t^{\prime}$ and $\phi^{\prime}$ define the position of the source, which are shown in Figure 13.

$s^{d}$ is the distance between the diffraction and the observation point, which is given by

$$
s^{d}=r_{o}+(r-a) \sin \theta \cos \phi^{\prime}
$$

$r_{o}$ is the distance from the center of the ogive to the observation point, and finally

$\xi$ is given by

$$
\begin{gathered}
\xi=\int_{Q^{\prime}}^{Q} d t^{\prime} \frac{m\left(t^{\prime}\right)}{\rho_{g}\left(t^{\prime}\right)}, \text { and } \\
m\left(Q^{\prime}\right)=\left[\frac{k \rho_{g}\left(Q^{\prime}\right)}{2}\right]^{1 / 3}
\end{gathered}
$$

Using this approach, the shadow region field can be written as

$$
\bar{E}^{s}(P)=\int_{-L / 2}^{L / 2} \int_{\phi_{b}}^{2 \pi-\phi_{b}} \bar{F}\left(\phi^{\prime}, t^{\prime}\right) e^{j \kappa \Phi\left(\phi^{\prime}\right)} e^{j t^{\prime} \beta} e^{-j k r_{o}} d \phi^{\prime} d t^{\prime}
$$

where

$$
\bar{F}\left(\phi^{\prime}, t^{\prime}\right)=\frac{-j k T}{4 \pi} a \bar{J}_{1}\left(\phi^{\prime}, t^{\prime}\right) Z_{o} H(\xi) \sqrt{\frac{d \psi_{o}}{\rho_{2}^{d} d \psi} \sqrt{\frac{\rho_{2}^{d}}{s^{d}\left(\rho_{2}^{d}+s^{d}\right)}}}
$$




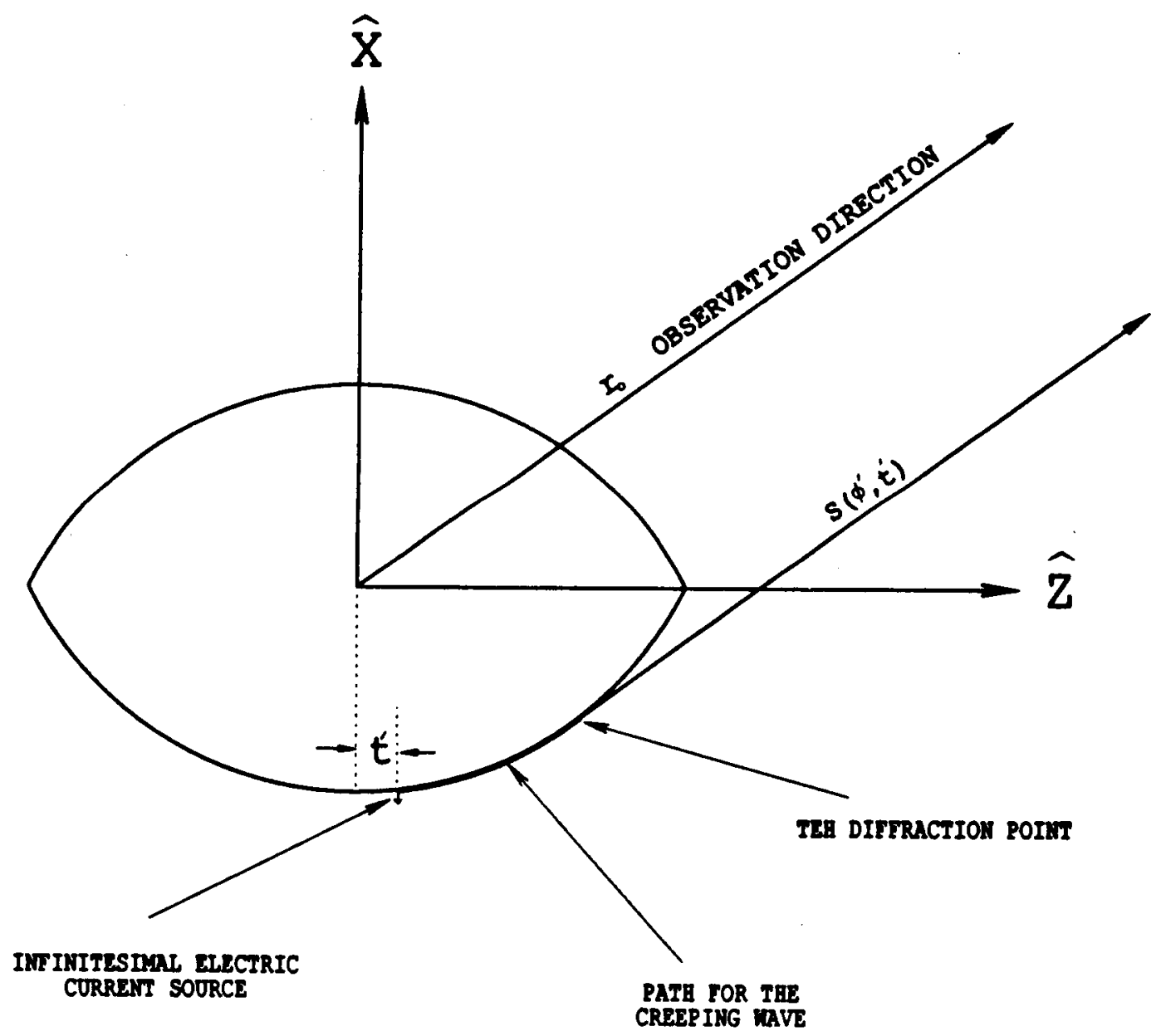

Figure 13: Creeping wave radiation from a source mounted on an ogive. 


$$
\begin{gathered}
\kappa=2(r \theta k-k(r-a) \sin \theta) \\
\Phi\left(\phi^{\prime}\right)=\cos \phi^{\prime} \\
\beta=k, \text { and } \\
\bar{J}_{1}\left(\phi^{\prime}, t^{\prime}\right)=\bar{J}\left(\phi^{\prime}, t^{\prime}\right) e^{-j(r \theta k-k(r-a) \sin \theta) .}
\end{gathered}
$$

The function $H(\xi)$ has a phase dependence on $\phi^{\prime}$; however, it is small, and it will not affect the stationary phase integration result.

Now, let us use the stationary phase integration method to evaluate the integral in Equation (3.24) with respect to $\phi^{\prime}$. First, one finds the stationary phase point using the following expressions:

$$
\begin{gathered}
\Phi\left(\phi^{\prime}\right)=\cos \phi^{\prime} \\
\Phi^{\prime}\left(\phi^{\prime}\right)=-\sin \phi^{\prime}, \text { and }
\end{gathered}
$$

and

$$
\Phi^{\prime \prime}\left(\phi^{\prime}\right)=-\cos \phi^{\prime}
$$

The stationary point is at $\Phi^{\prime}\left(\phi_{s t}\right)=0$ which means that $\phi_{s t}=0, \pi$. But since the shadowed part of the strap is being studied here, $\phi_{s t}=\pi$. Then, one finds that

$$
\bar{E}^{s}(p)=\int_{-L / 2}^{L / 2} \bar{F}\left(\pi, t^{\prime}\right) \sqrt{\frac{2 \pi}{\kappa\left|\Phi^{\prime \prime}(\pi)\right|}} e^{j\left[\kappa \Phi(\pi)+\frac{\pi}{4} \operatorname{sgn}\left(\Phi^{\prime \prime}(\pi)\right)\right]} e^{j t^{\prime} \beta^{-j k r_{o}}} d t^{\prime}
$$


which can be written as

$$
\bar{E}^{s}(p)=\sqrt{\frac{2 \pi}{\kappa}} e^{j\left(-\kappa+\frac{\pi}{4}\right)} e^{-j k r_{o}} \int_{-L / 2}^{L / 2} \bar{F}\left(\pi, t^{\prime}\right) e^{j t^{\prime} \beta} d t^{\prime} .
$$

The above integral is then evaluated numerically.

The current $\bar{J}\left(\phi^{\prime}, t^{\prime}\right)$ is found using the moment method approach described in Section 2.2. In using that method, one needs to find the excitation for each cell in the strap; this can be done as follows. Consider an electric current moment located at the bottom of the ogive radiating in free space as shown in Figure 12. According to Equations (2.5), (2.19) and (3.23a), the electric field due to a current moment at the stationary point and an observation point located in the $X Z$ plane is given by

$$
d \bar{E}\left(p_{s}\right) \sim C d p\left(Q^{\prime}\right) Z_{o} H(\xi) \sqrt{\frac{d \psi_{o}}{\rho_{2}^{d} d \psi}} d^{-j k t} \sqrt{\frac{\rho_{2}^{d}}{s^{d}\left(\rho_{2}^{d}+s^{d}\right)}} e^{-j k s^{d}} \hat{n} .
$$

In the far field, the above equation can be written as

$$
d \bar{E}\left(p_{s}\right) \sim C d p\left(Q^{\prime}\right) Z_{o} H(\xi) \sqrt{\frac{d \psi_{o}}{d \psi}} e^{-j k t} \frac{1}{s^{d}} e^{-j k s^{d}} \hat{n}
$$

with

$$
\begin{gathered}
t=r \theta-t^{\prime}, \text { and } \\
s^{d}=r_{o}-(r-a) \sin \theta .
\end{gathered}
$$

One can use reciprocity to find the electric field at the location of the electric current moment due to a plane wave incidence. It is found to be given by

$$
H(\xi) \sqrt{\frac{d \psi_{o}}{d \psi}} \epsilon^{-j k t^{\prime}} \epsilon^{j(-r \theta k+k(r-a) \sin \theta)}
$$


The total backscattered electric field is then given by

$$
\bar{E}(P)=\bar{E}^{u}(P)+\bar{E}^{s}(P)
$$

In Equations (3.21) and (3.28), one can see that, when $\theta=0^{\circ}$, the field becomes singular which means that the equation is not valid at or near that caustic direction. This is true because at $\theta=0^{\circ}$, all the current elements around the strap contribute to the backscattered field. In order to find the field at or near this caustic direction, one needs to integrate the current around the whole strap. This can be done as follows. When $\theta=0^{\circ}$ the electric field radiated by the current in the strap can be written as

$$
\bar{E}(p)=\frac{-j k}{4 \pi} Z_{o} H^{L}(0) T \int_{-L / 2}^{L / 2} \int_{0}^{2 \pi} J\left(\phi^{\prime}, t^{\prime}\right) \hat{n}_{L} \frac{e^{-j k R}}{R} a d \phi^{\prime} d t^{\prime}
$$

Let $R=r_{o}-t^{\prime} ;$ then, one finds that

$$
\bar{E}(p)=\frac{-j k}{4 \pi r_{o}} Z_{o} T a H^{L}(0) e^{-j k r_{o}} \int_{-L / 2}^{L / 2} \int_{0}^{2 \pi} J\left(\phi^{\prime}, t^{\prime}\right) \hat{n}_{L} e^{j k t^{\prime}} d \phi^{\prime} d t^{\prime}
$$

From the symmetry of the structure one can see that the backscattered electric field in $\hat{x}$ direction can be written as

$$
\bar{E}(p)=\frac{-j k}{4 \pi r_{o}} Z_{o} T a H^{L}(0) e^{-j k r_{0}} \int_{-L / 2}^{L / 2} \int_{0}^{2 \pi} J\left(\phi^{\prime}, t^{\prime}\right) \cos \left(\phi^{\prime}\right) e^{j k t^{\prime}} d \phi^{\prime} d t^{\prime} \hat{x}
$$

Also, one can easily see that $J\left(\phi^{\prime}, t^{\prime}\right)$ can be written as

$$
J\left(\phi^{\prime}, t^{\prime}\right)=J\left(0, t^{\prime}\right) \cos \left(\phi^{\prime}\right)
$$

Thus, one obtains that

$$
\bar{E}(p)=\frac{-j k}{4 \pi r_{o}} Z_{o} T a H^{L}(0) e^{-j k r_{o}} \int_{-L / 2}^{L / 2} \int_{0}^{2 \pi} J\left(0, t^{\prime}\right) \cos ^{2}\left(\phi^{\prime}\right) e^{j k t^{\prime}} d \phi^{\prime} d t^{\prime} \hat{x}
$$

and

$$
\bar{E}(p)=\frac{-j k}{4 r_{o}} Z_{o} T a H^{L}(0) e^{-j k r_{o}} \int_{-L / 2}^{L / 2} J\left(0, t^{\prime}\right) e^{j k t^{\prime}} d t^{\prime} \hat{x}
$$


The above integral can again be evaluated numerically across the width of the strap.

Let us now justify the claim stated at the beginning, the backscattered $\mathrm{H}$ plane pattern is very low and does not need to be considered. The backscattered field from the strap described in equations (3.21) and (3.28) depends on the electric current at the stationary points. That current is a function of the incident electric field at the stationary points. In the H-plane backscattered field case, the stationary points are at $\phi^{\prime}=\pi / 2$ and $\phi^{\prime}=-\pi / 2$. The electric field is very weak at these stationary points because the incident electric field is tangent to the surface of the, and the strap is very thin. This means that the electric current at $\phi^{\prime}=\pi / 2$ and $\phi^{\prime}=-\pi / 2$ is almost zero, hence the backscattered field in the H-plane is very low.

In this chapter, the method presented in Chapter II was used to solve for the backscattered fields from a strap surrounding a structure. This technique was applied to an ogive simply to provide results for a specific case. The method split the solution into two parts. One part corresponds to the backscattered field from the lit part of the strap, and the other one corresponds to backscattered field from the shadowed part of the strap. The above method does not work at or near $\theta=0^{\circ}$, because it is a caustic for the backscattered field such that stationary phase dose not apply. Therefore, at that point the backscattered field was evaluated by integrating the current over the whole strap. In the next chapter some computed and measured results are presented. 


\section{CHAPTER IV}

\section{RESULTS AND EMPIRICAL DESIGN FORMULAS}

\subsection{Measured and computed results}

In this section, the results of chapter III are used to compute the E-plane RCS of a strap wrapped around an ogive as shown in Figure 14. First, some computed and measured results for a particular strap are compared in order to demonstrate the validity of this theoretical approach. Then, some individual computed terms are presented to demonstrate the way in which the solution works.

Figure 15 shows the RCS of a dielectric strap wrapped around an ogive versus frequency for nose-on incidence $\left(\theta=0^{\circ}\right)$. The strap has $L=2^{\prime \prime}, T=0.004^{\prime \prime}$ and $\epsilon_{r}=2.3$. The dimensions of the ogive are $a=2.37^{\prime \prime}$ and $b=18^{\prime \prime}$. Note that at $4 \mathrm{GHz}$ the RCS of the strap is about $-65 \mathrm{dBsm}$. The RCS increases with the increase in frequency; however, it is still low at high frequencies. In Figure 15, the measured RCS is also shown which show reasonable agreement with the calculated ones. To measure the RCS of the strap, it was wrapped around an ogive and mounted in the OSU compact range facility as shown in Figure 16. First, the RCS of the whole structure was measured from 2 to $18 \mathrm{GHz}$ every $10 \mathrm{MHz}$. Next the phase center was taken to be at the middle of the strap; then smoothing was used to isolate the RCS of the strap. The measured result which appears in Figure 15 was obtained using a 201 point smoothing. A 101 points smoothing gives the result shown in figure 17 . One can see that the 201 point smoothing gives the most 


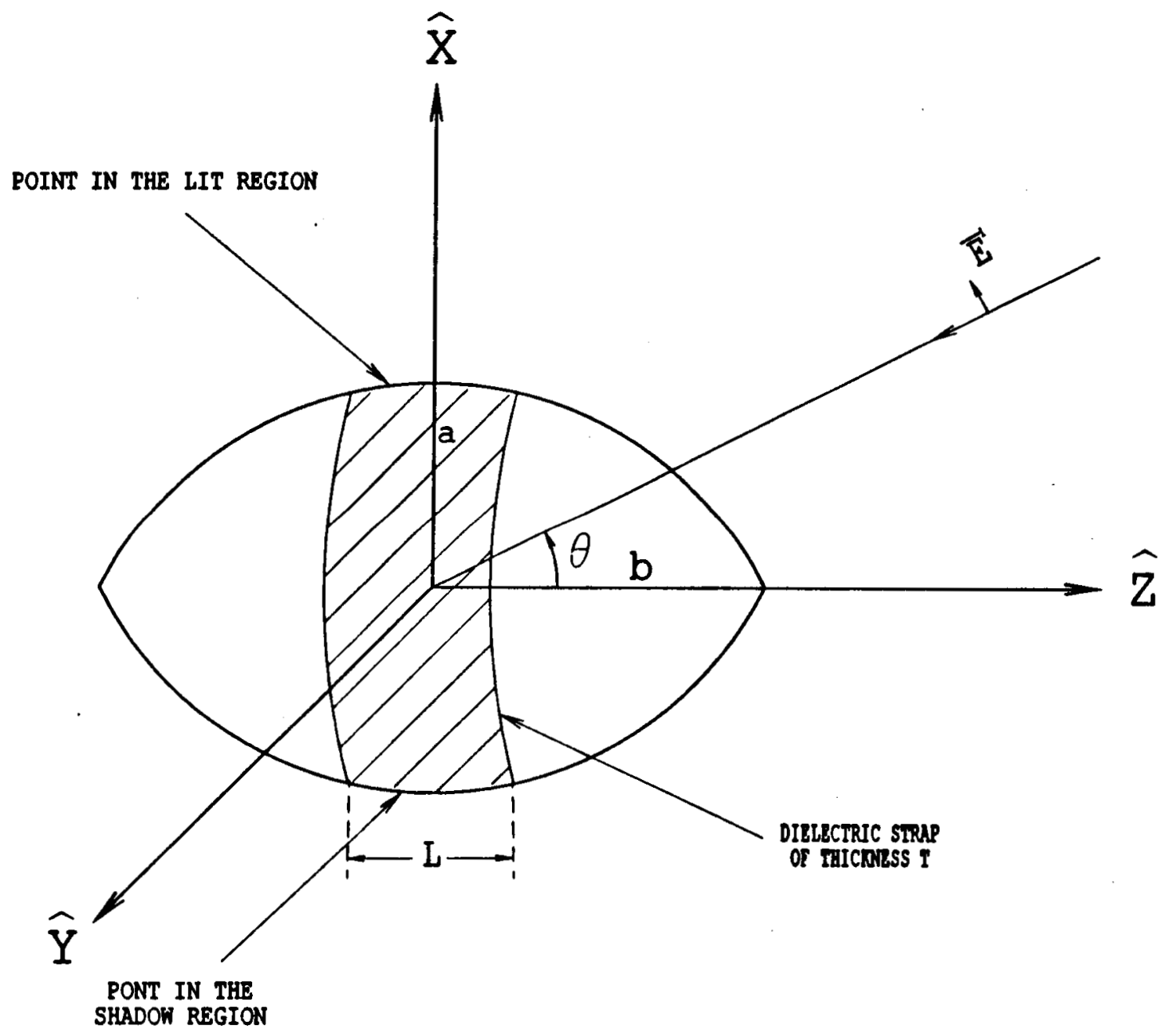

Figure 14: A dielectric strap wrapped around an ogive. 


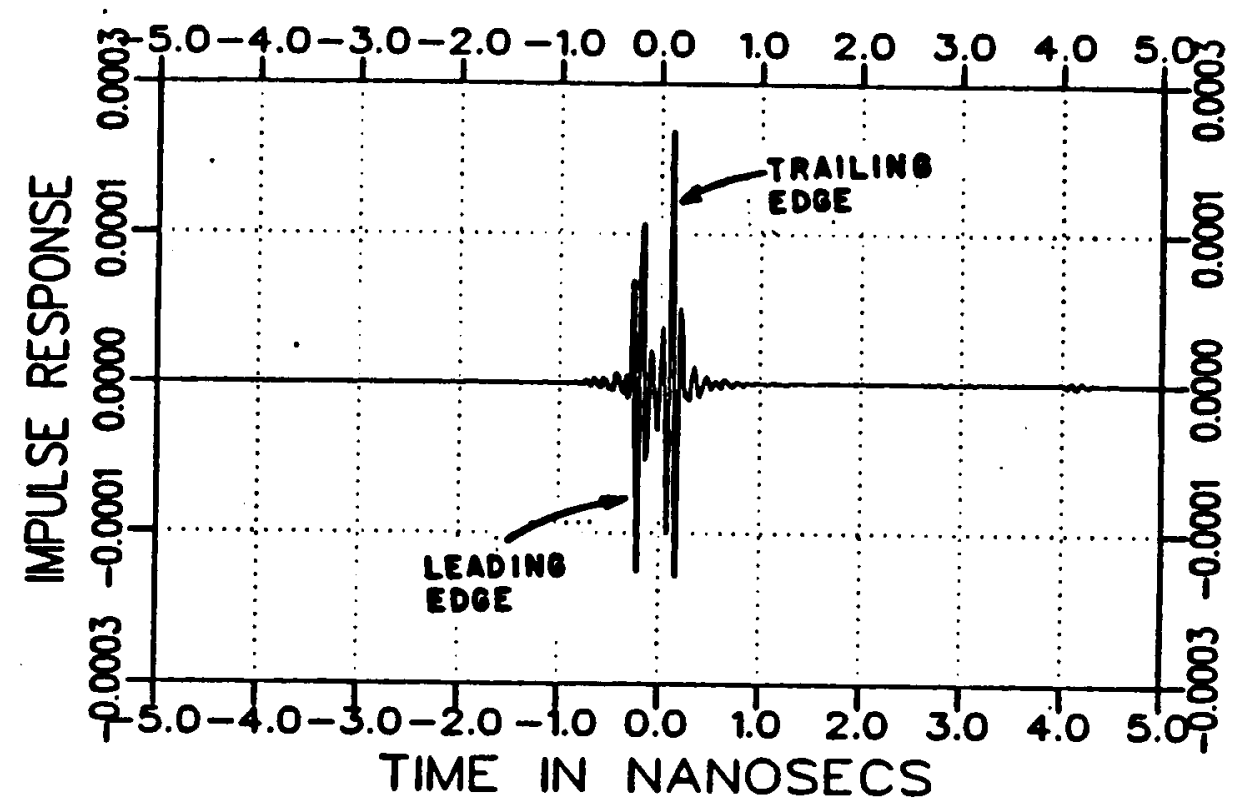

(a)Measured Time Domain

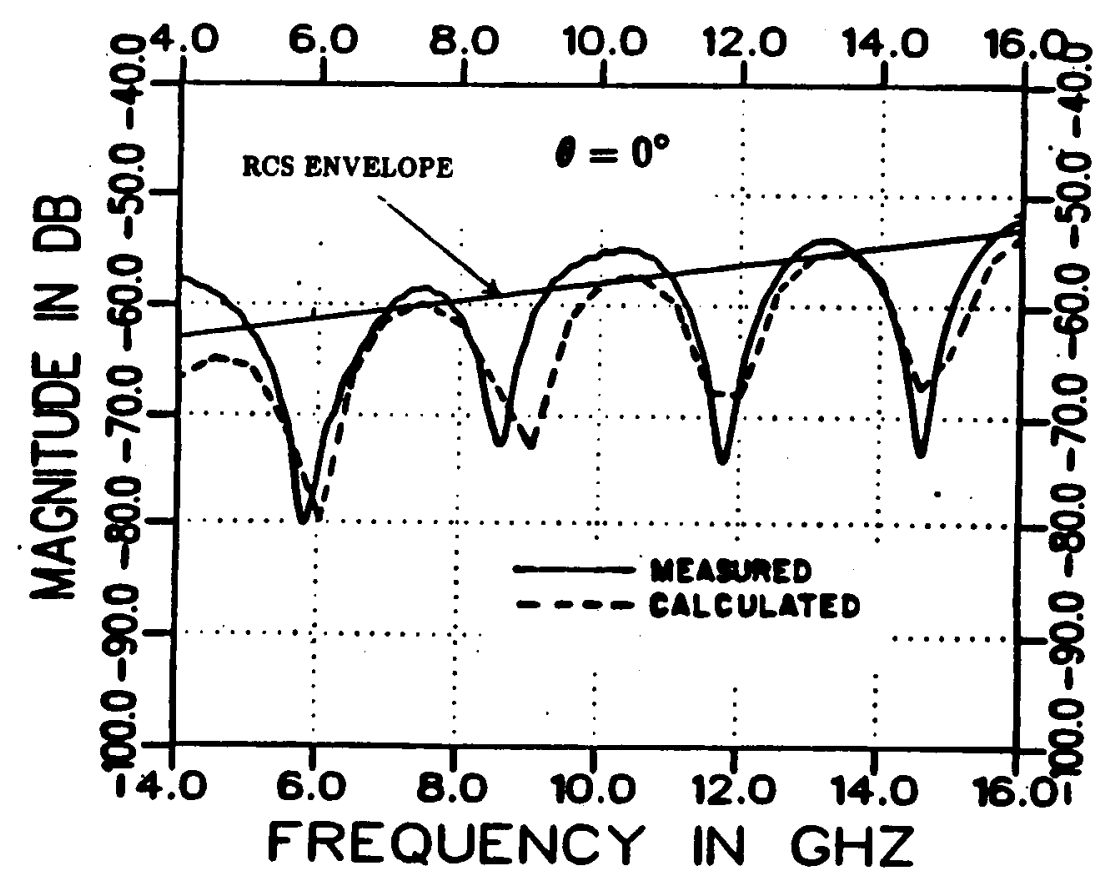

(b) Frequency Domain

Figure 15: Nose-on RCS of a strap versus frequency with 201 point smoothing. $\mathrm{a}=2.37^{\prime \prime}, \mathrm{b}=18.0^{\prime \prime}, \mathrm{L}=2^{\prime \prime}, \mathrm{T}=0.004^{\prime \prime}$ and $\epsilon_{r}=2.3$. 


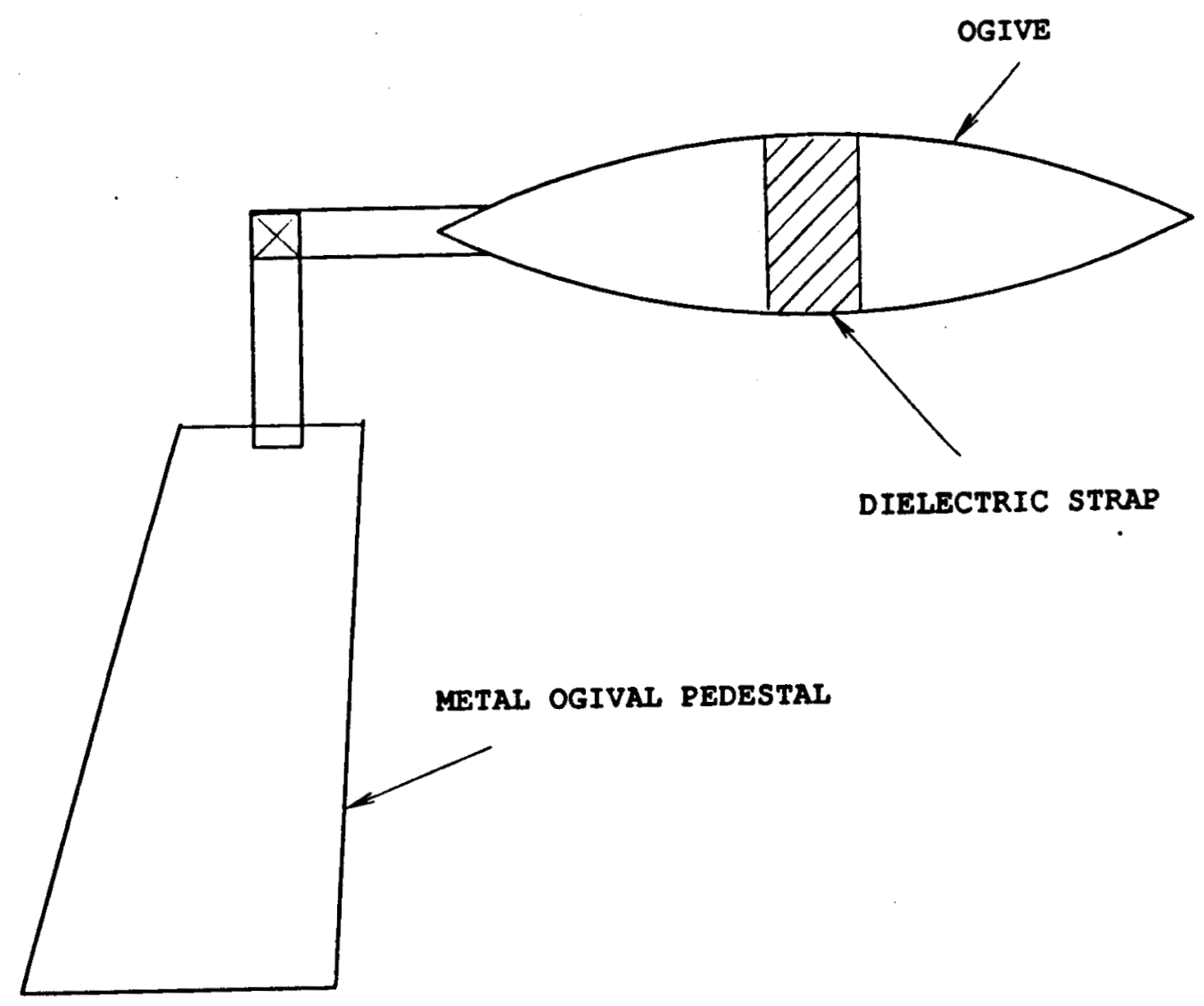

Figure 16: The measurement set up. 


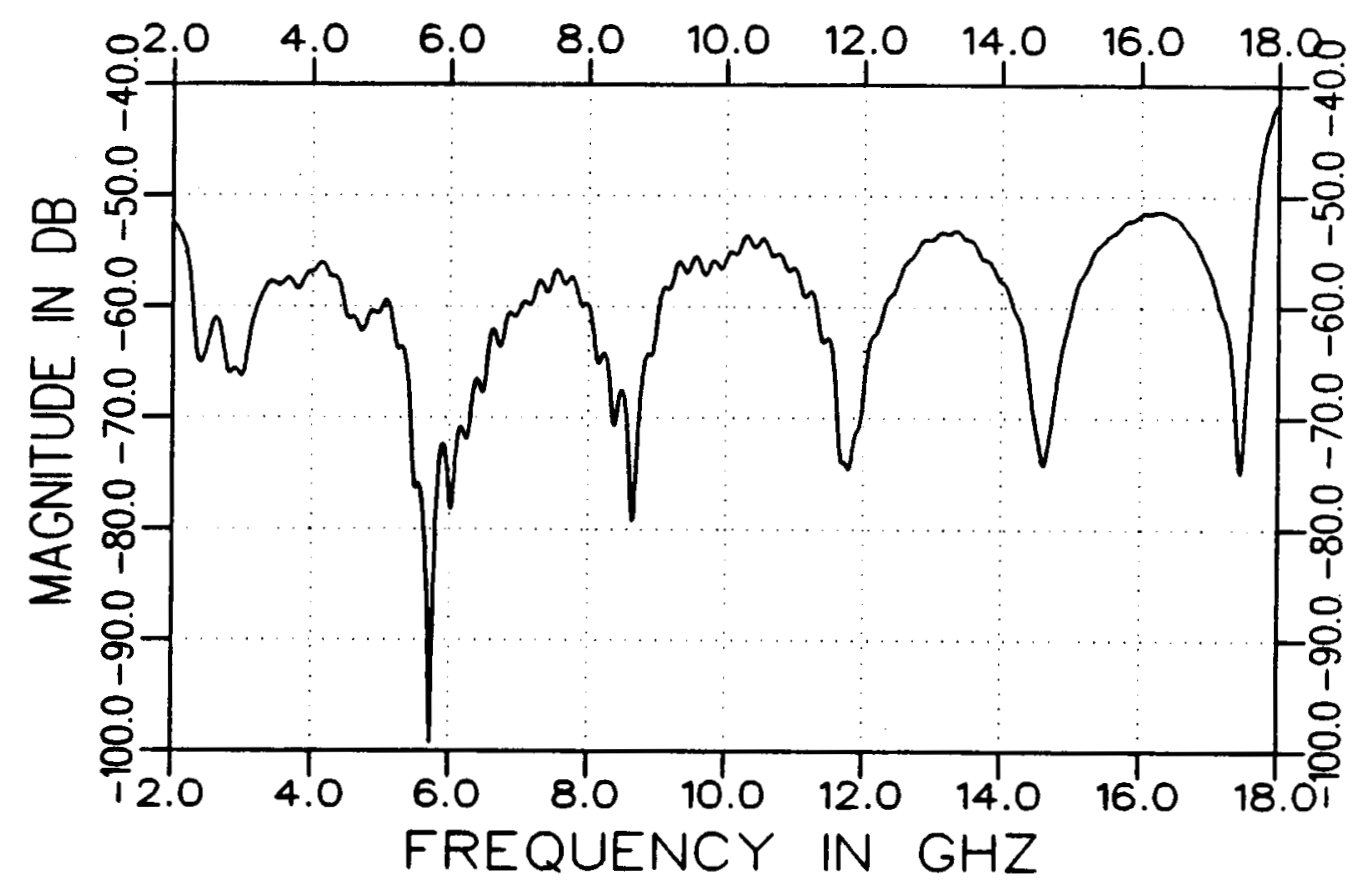

Figure 17: Nose-on RCS of a strap versus frequency with 101 point smoothing. $\mathrm{a}=2.37^{\prime \prime}, \mathrm{b}=18.0^{\prime \prime}, \mathrm{L}=2^{\prime \prime}, \mathrm{T}=0.004^{\prime \prime}$ and $\epsilon_{r}=2.3$. 
reasonable results. Therefore, the rest of the measured results are presented using a 201 point smoothing. The time domain results obtained from the measured data is shown in Figure 15(a). It indicates that the strap scattering is dominated by the front and back edges. Note that the two edges of the strap scatter roughly the same level, which causes the lobe structure observed in the frequency domain.

Figure 18 shows the measured as well as the computed RCS of the tip of the ogive. The computed RCS of the tip of the ogive was found by using the following physical optics formula:

$$
\sigma(0)=\frac{\pi}{4 k^{2}} \tan ^{4}(\alpha)
$$

where $\alpha$ is the half cone angle of the ogive. One can note that the measured RCS does not agree with the computed one near the upper and lower ends of the frequency spectrum because the measured result was obtained using the smoothing approach mentioned earlier. Note that smoothing does not work well at the ends of the spectrum because one can't center the smooth data around the ends. In this case, the end values are found using 101 points to the right or left of the end point.

Figures 19 through 26 show the computed as well as measured RCS of the strap versus frequency for observation angles from 5 to 40 degrees with an increment of 5 degrees. The quality of the measured results decreases with the increase in the observation angle, $\theta$, in that as $\theta$ increases the return from the strap gets smaller and smaller. This make it hard to separate the RCS of the strap from the noise. For Figures 19 through 26, the RCS was computed without including the contribution from the shadowed part of the strap in that the cylinder perturbation method does not work well for thin ogives, such as the one used for the measurement. However, this did not have any considerable effect on the results. This point well be discussed 


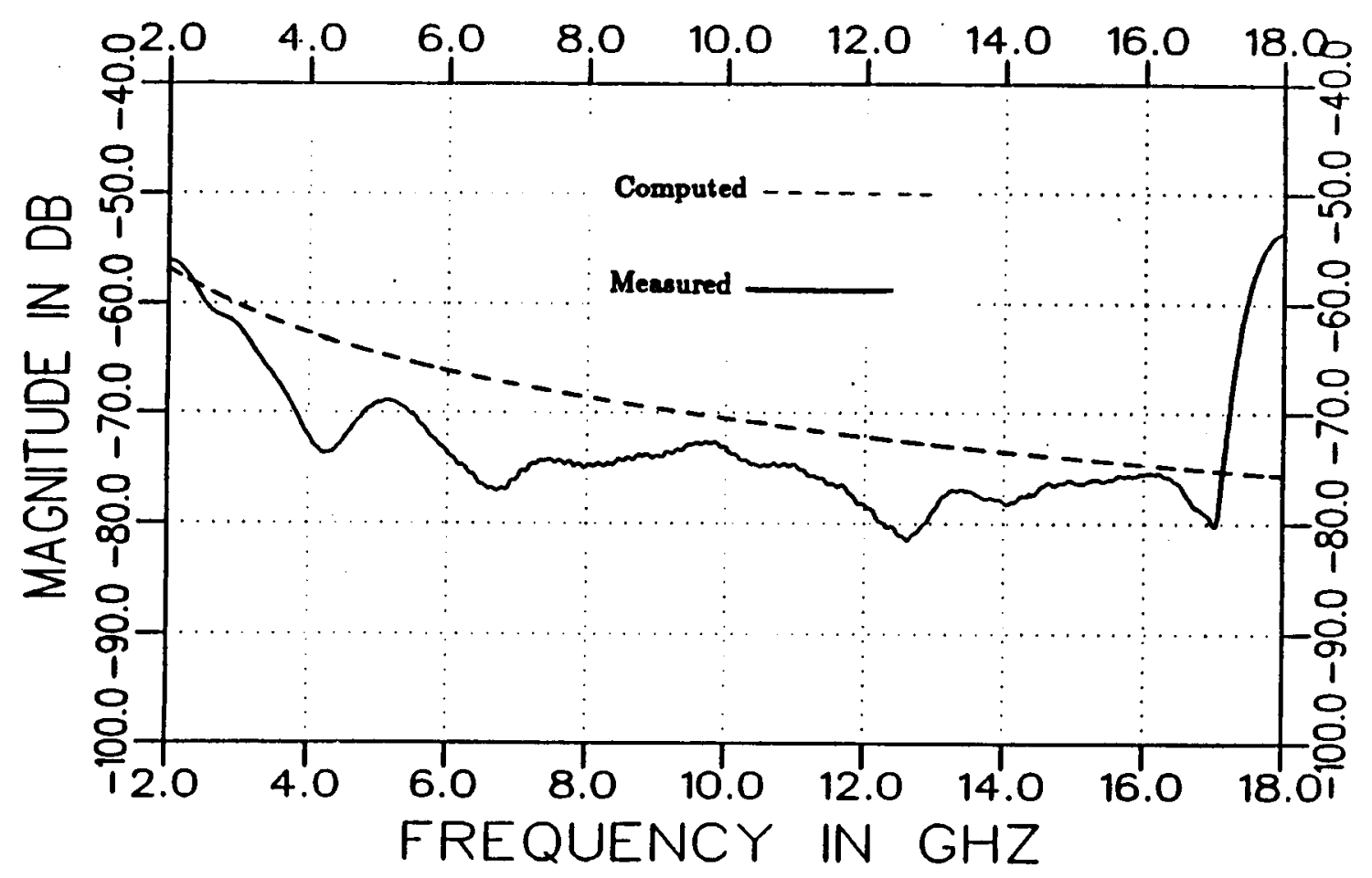

Figure 18: Nose-on RCS of the tip of the ogive versus frequency with $a=2.37^{\prime \prime}$ and $b=18.0^{\prime \prime}$.

later in this section. Although the computed results in Figures 19 through 26 were found using this simple approach, reasonable agreement was obtained in each case.

In order to demonstrate how the solution works, some computed results for different straps are presented. Figure 28 shows the backscattered field from a dielectric strap over a ground plane, with the geometry shown in Figure 27. The backscattered field is computed at $6 \mathrm{GHz}$, and the dimensions of the strap are $L=2.5^{\prime \prime}, T=0.004^{\prime \prime}$ and $\epsilon_{r}=4.0$. The null which appears at $\theta=90^{\circ}$ is expected for a thin strap because the incident electric field and its image field cancel at the ground plane, which means that the strap is not significantly excited and hence does not backscatter significantly. A similar null is expected in the RCS pattern of the strap wrapped around the ogive.

Figures 29 through 31 are for an ogive with dimensions, $a=14.76^{\prime \prime}$ and $b=29.53^{\prime \prime}$. Figure 29 shows the RCS of a strap with $L=3.69^{\prime \prime}, T=0.008^{\prime \prime}$ and 


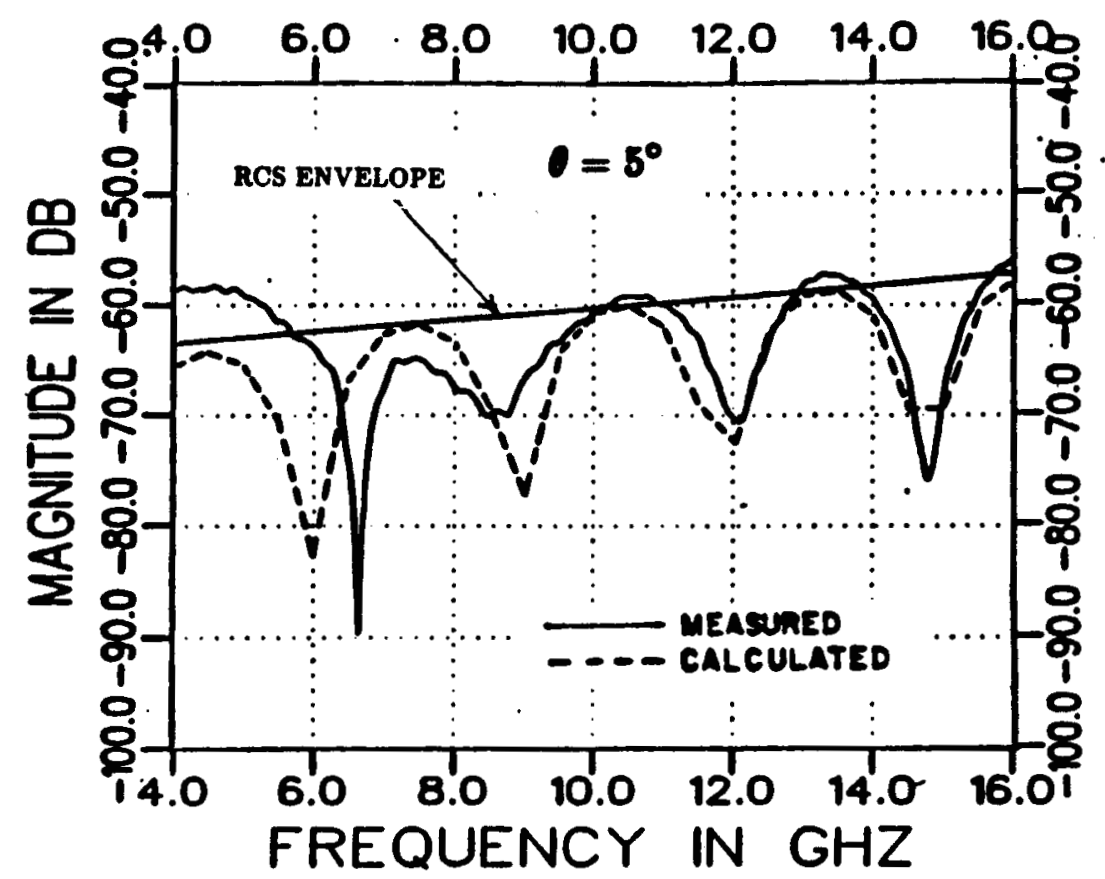

Figure 19: RCS of a strap versus frequency with $a=2.37^{\prime \prime}, b=18.0^{\prime \prime}, L=2^{\prime \prime}$, $\mathrm{T}=0.004^{\prime \prime}$ and $\epsilon_{r}=2.3$.

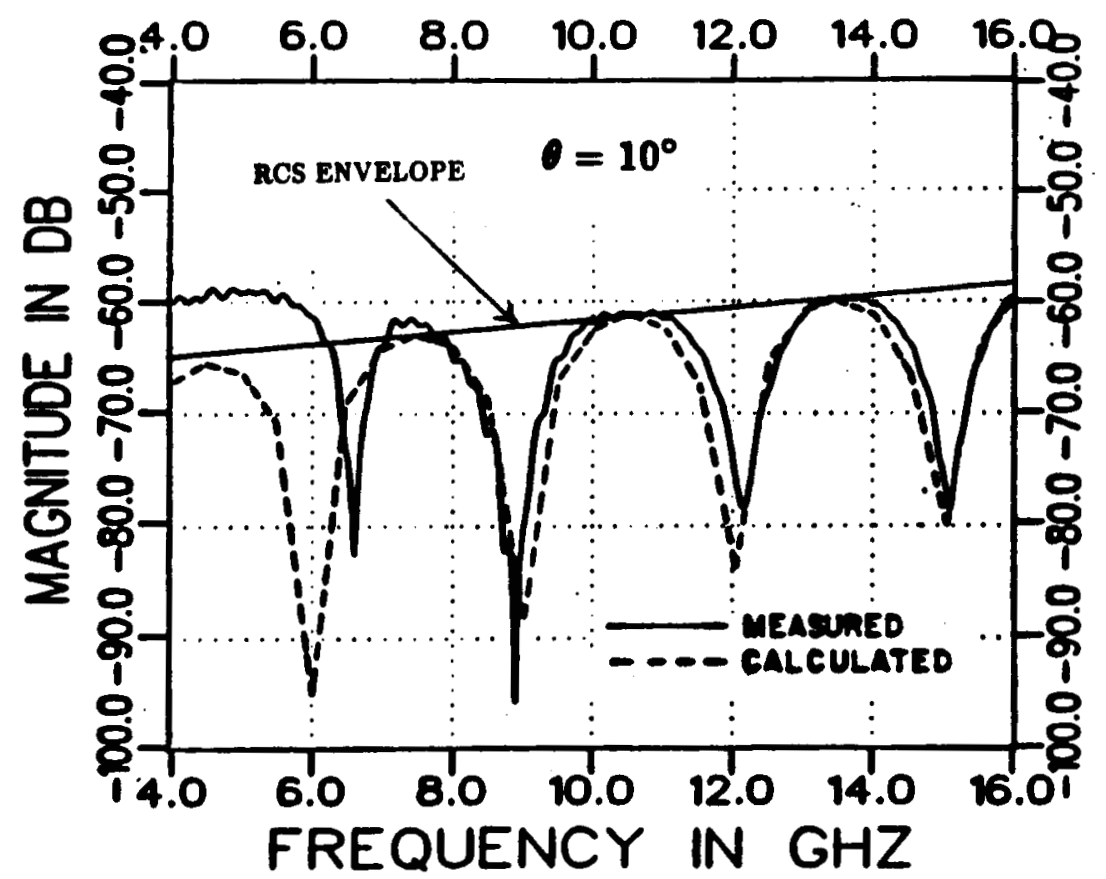

Figure 20: RCS of a strap versus frequency with $a=2.37^{\prime \prime}, b=18.0^{\prime \prime}, L=2^{\prime \prime}$, $\mathrm{T}=0.004^{\prime \prime}$ and $\epsilon_{r}=2.3$. 


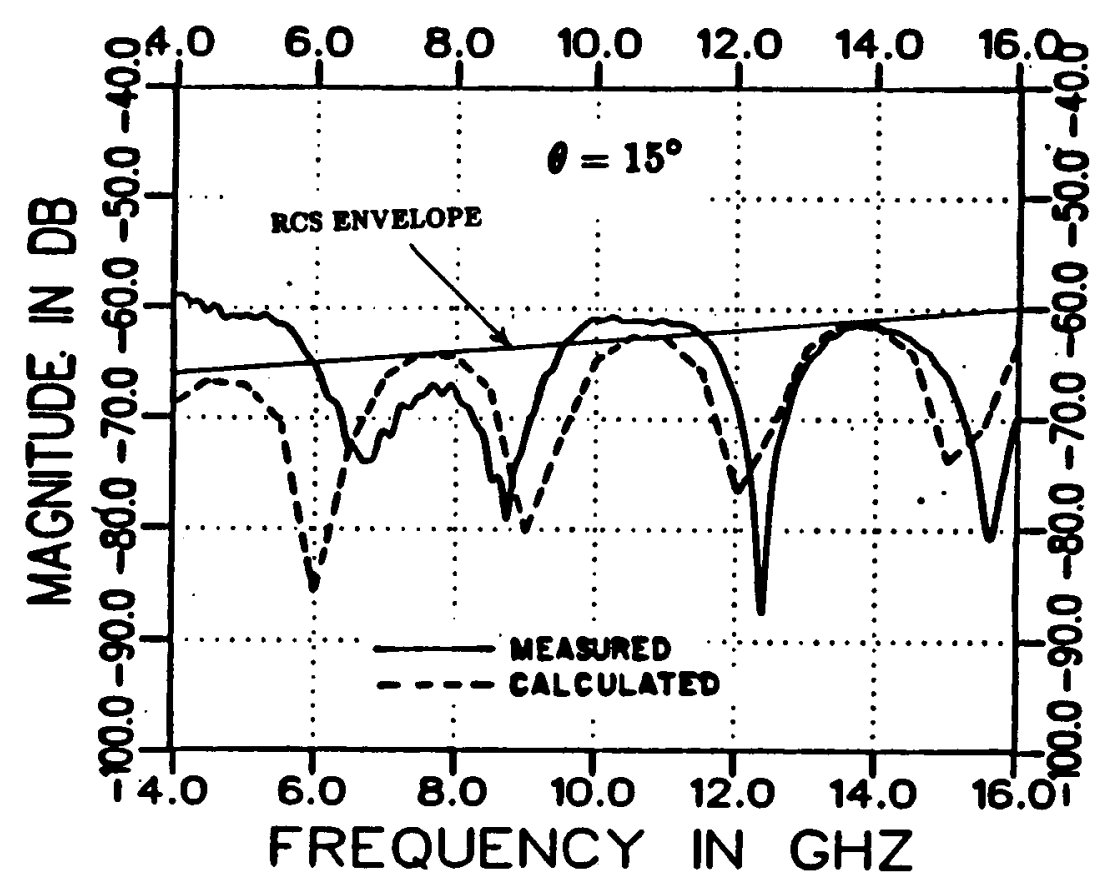

Figure 21: RCS of a strap versus frequency with $a=2.37^{\prime \prime}, b=18.0^{\prime \prime}, L=2^{\prime \prime}$, $\mathrm{T}=0.004^{\prime \prime}$ and $\epsilon_{r}=2.3$.

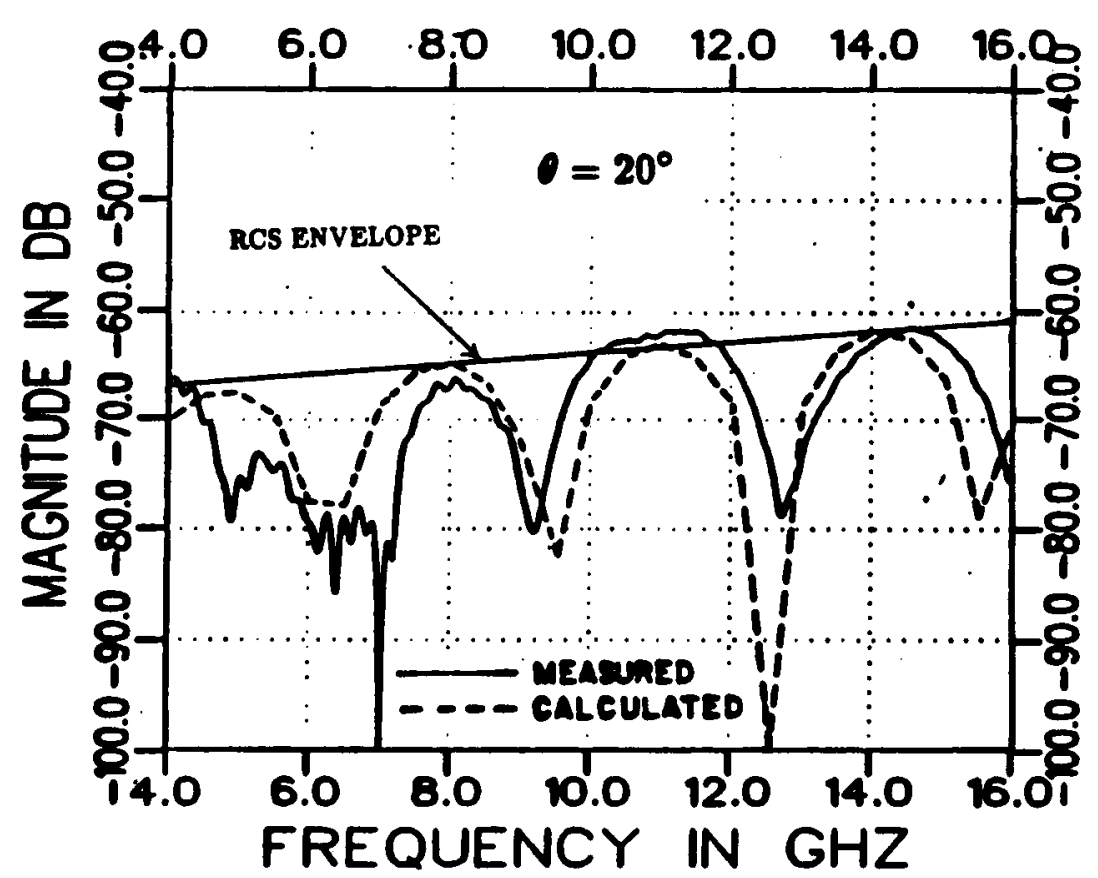

Figure 22: RCS of a strap versus frequency with $a=2.37^{\prime \prime}, b=18.0^{\prime \prime}, L=2^{\prime \prime}$, $\mathrm{T}=0.004^{\prime \prime}$ and $\epsilon_{r}=2.3$. 


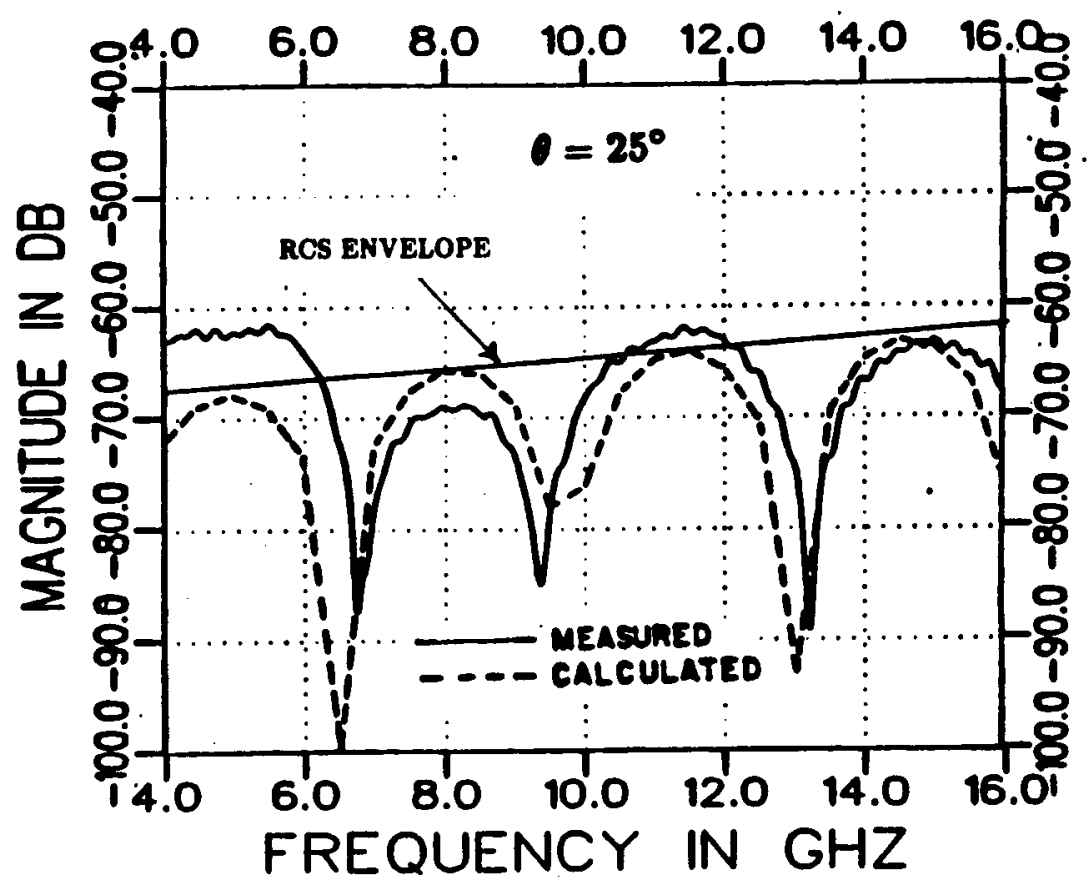

Figure 23: RCS of a strap versus frequency with $a=2.37^{\prime \prime}, b=18.0^{\prime \prime}, L=2^{\prime \prime}$, $\mathrm{T}=0.004^{\prime \prime}$ and $\epsilon_{r}=2.3$.

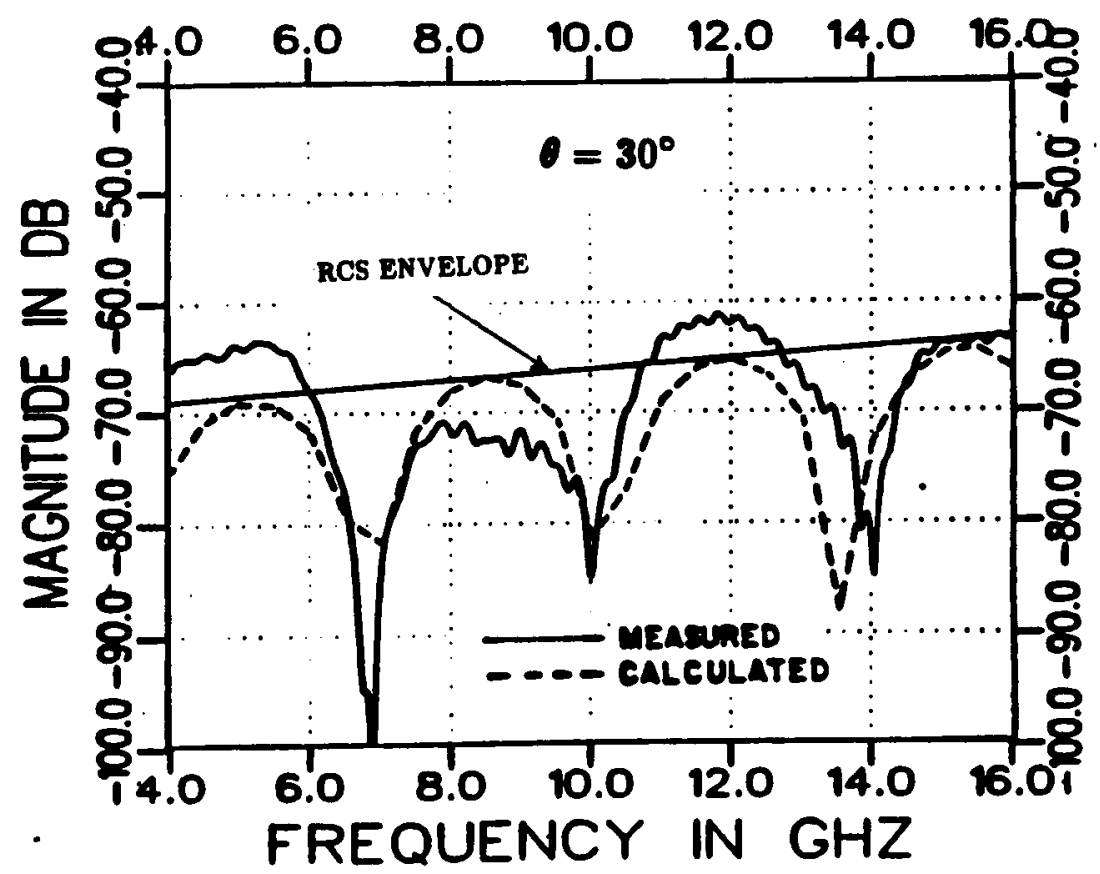

Figure 24: RCS of a strap versus frequency with $\mathrm{a}=2.37^{\prime \prime}, \mathrm{b}=18.0^{\prime \prime}, \mathrm{L}=2^{\prime \prime}$, $\mathrm{T}=0.004^{\prime \prime}$ and $\epsilon_{r}=2.3$. 


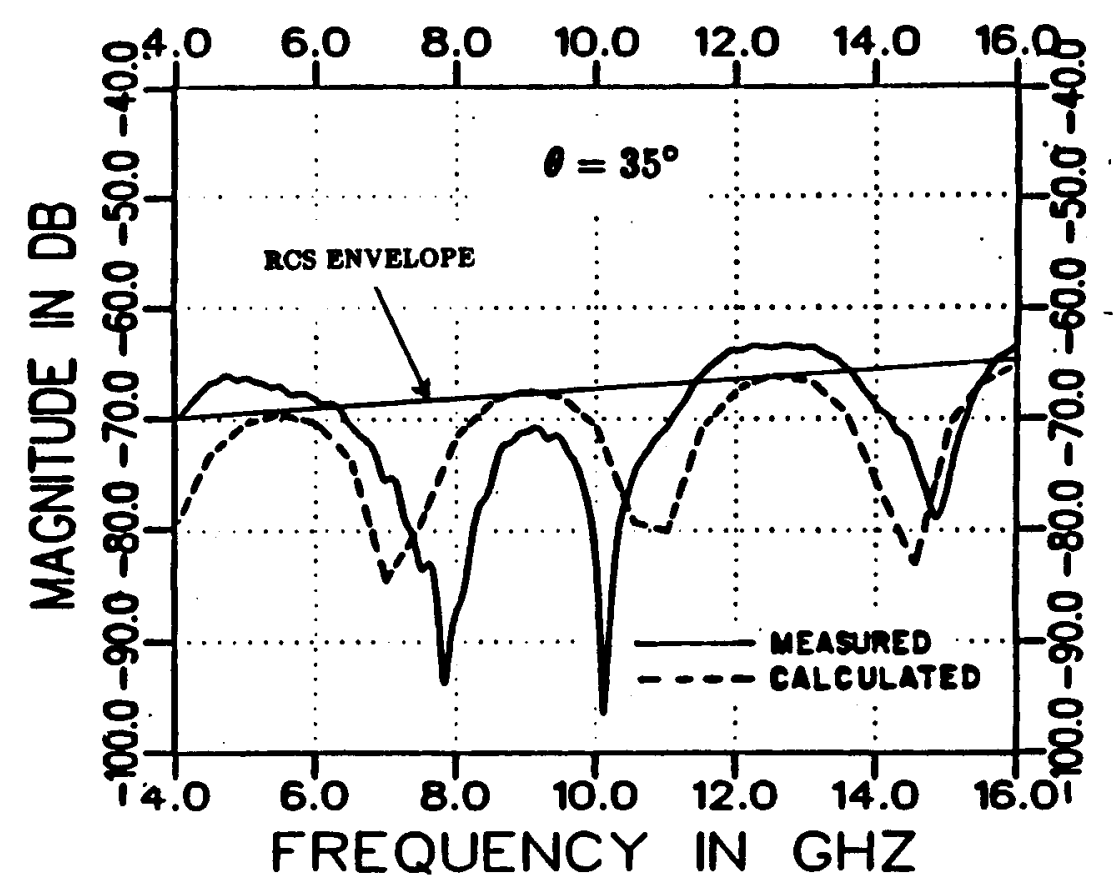

Figure 25: RCS of a strap versus frequency with $a=2.37^{\prime \prime}, b=18.0^{\prime \prime}, \mathrm{L}=2^{\prime \prime}$, $\mathrm{T}=0.004^{\prime \prime}$ and $\epsilon_{r}=2.3$.

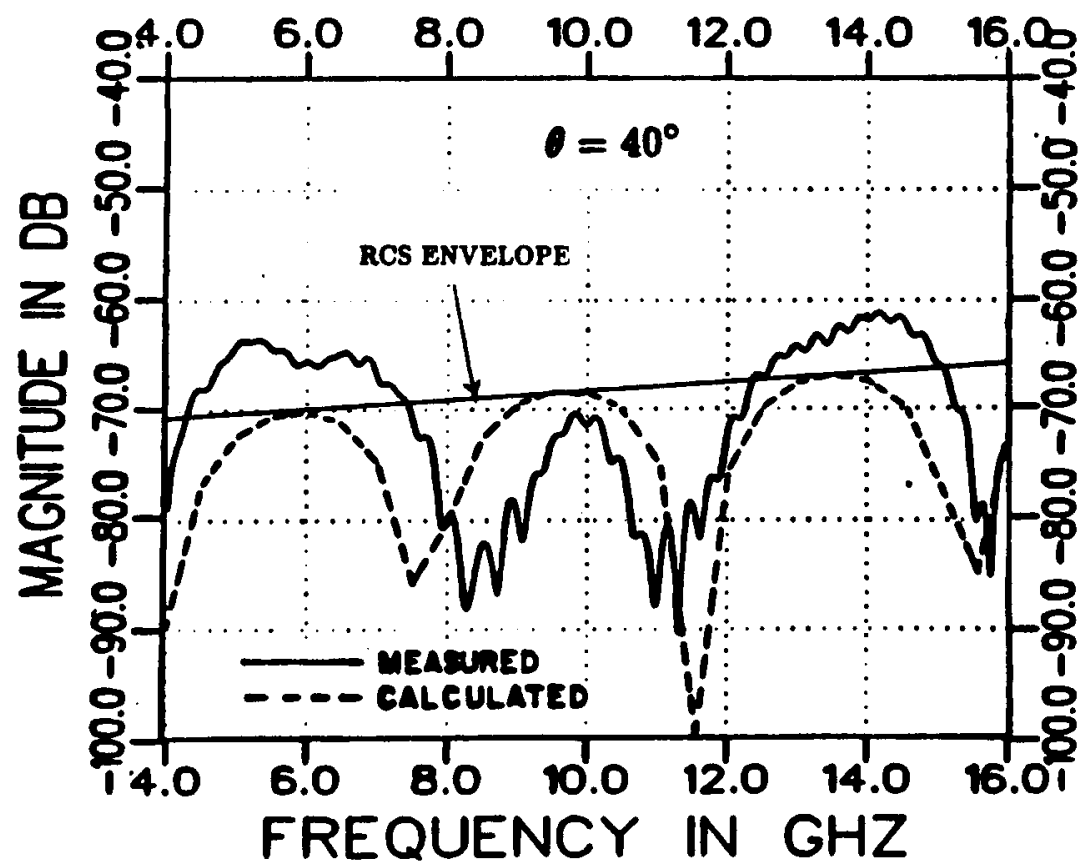

Figure 26: RCS of a strap versus frequency with $a=2.37^{\prime \prime}, b=18.0^{\prime \prime}, \mathrm{L}=2^{\prime \prime}$, $\mathrm{T}=0.004^{\prime \prime}$ and $\epsilon_{r}=2.3$. 


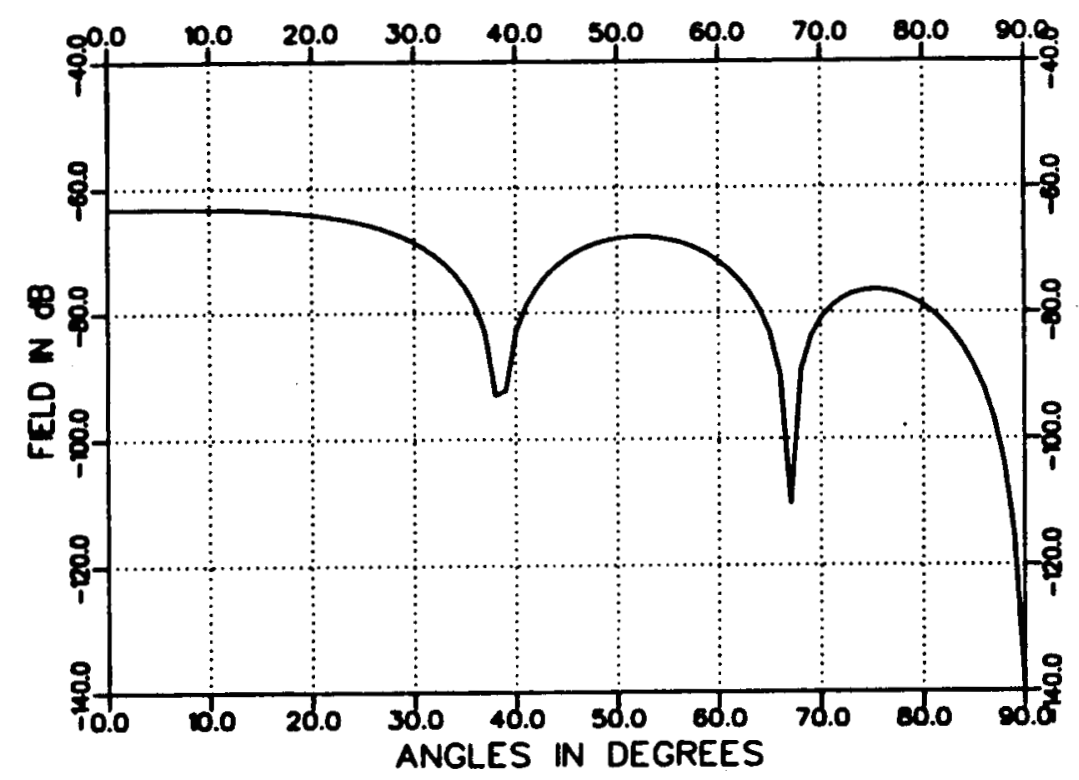

Figure 27: Back scattered field from dielectric strap over a ground plane with $\mathrm{L}=2.5^{\prime \prime}, \mathrm{T}=0.004^{\prime \prime}, \epsilon_{r}=4.0$ and $\mathrm{f}=6 \mathrm{GHz}$.

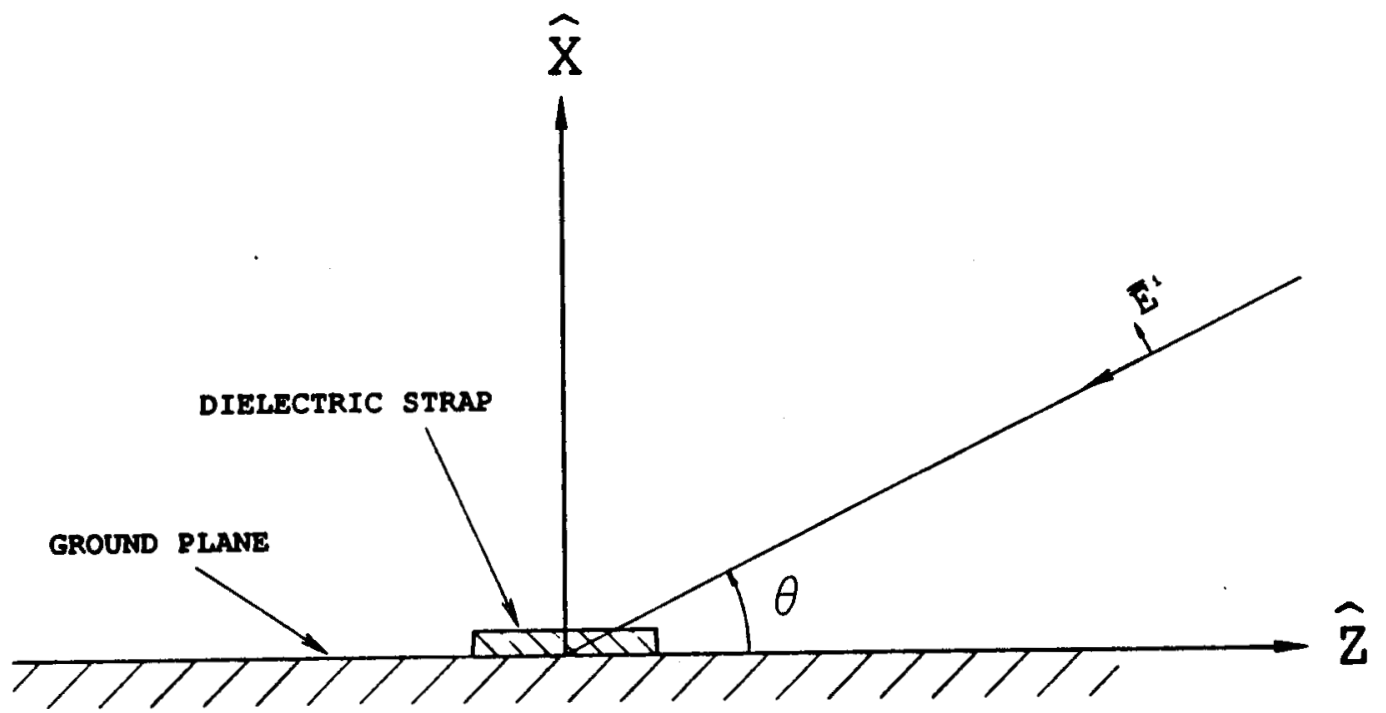

Figure 28: Dielectric strap over a ground plane. 
$0.004^{\prime \prime}$ and $\epsilon_{r}=4.0$ and 2.0. Figure 29(a) shows the RCS of the lit part of the strap; whereas, Figure 29(b) shows the RCS of the shadowed part of the strap. The total RCS of the strap is shown in Figure 29(c). Figure 30 shows the RCS of a strap with $L=2.36^{\prime \prime}, T=0.004^{\prime \prime}$ and $0.008^{\prime \prime}$, and $\epsilon_{r}=4.0$. As mentioned in chapter III, one can clearly see that the RCS of the lit and shadowed parts of the strap are not valid at or near $\theta=0^{\circ}$. Therefore, at $\theta=0^{\circ}$ the RCS of the strap is evaluated by integrating the current all around the strap. In the total RCS of the strap the, results are only shown outside the caustic region.

Figures 29 and 30 show that the RCS of the strap can be reduced by reducing the thickness and/or the dielectric constant of the strap. This means that a tradeoff can be made between the RCS of the strap and its physical properties, such as the maximum load that it can handle which is influenced by the thickness of the strap and its dielectric constant. An empirical formula which can help in making such a tradeoff is given in [2]. One can easily see that the nulls which appear in Figures 29 and 30 (except for the one at $\theta=90^{\circ}$ ) are due to the contributions from the front and back edges of the strap coming out of phase. Figures 29 and 30 also show that changing the width of the strap does not affect the RCS level but it affects the number of nulls appearing in the RCS pattern.

Figure 31 shows the nose-on RCS of the strap versus frequency. The RCS level of this strap is higher than the one in Figure 15, the main reason being that the dimension ' $a$ ' in the ogive of Figure 31 is equal to 14.76 "; while, ' $a$ ' in Figure 15 is equal to $2.37^{\prime \prime}$. This means that the strap of Figure 31 is much longer than the one of Figure 15, which explains the difference in the RCS level.

Figure 32 shows the RCS of the same strap used in the measurement (i.e. $L=2^{\prime \prime}, T=0.004^{\prime \prime} \epsilon_{r}=2.3, a=2.37^{\prime \prime}$ and $\left.b=18^{\prime \prime}\right)$ at a frequency of $4 \mathrm{GHz}$. 


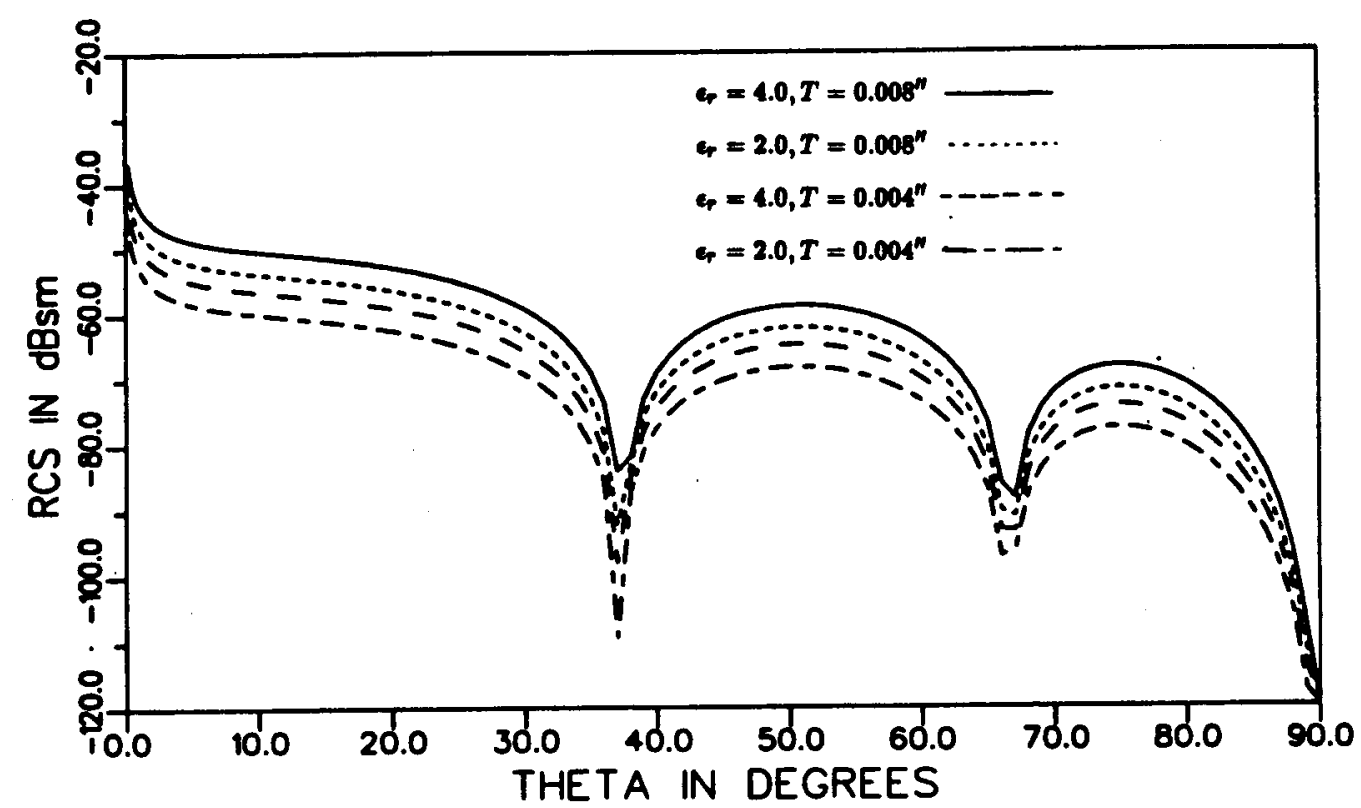

(a) RCS from the lit part of the strap.

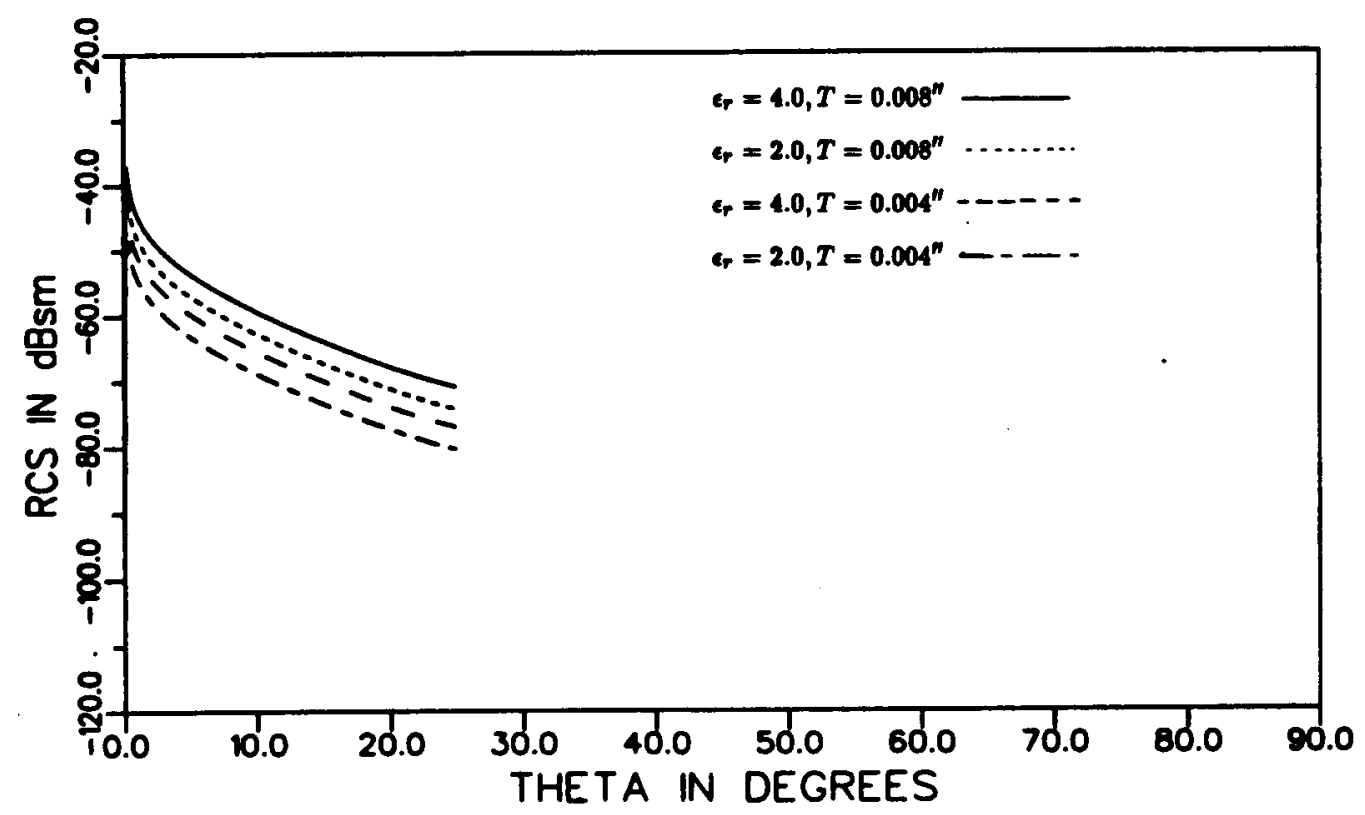

(b) RCS from the shadowed part of the strap.

Figure 29: RCS of a strap wrapped around an ogive with $a=14.76^{\prime \prime}, b=29.53^{\prime \prime}$, $\mathrm{L}=3.69^{\prime \prime}$ and $\mathrm{f}=4 \mathrm{GHz}$. 


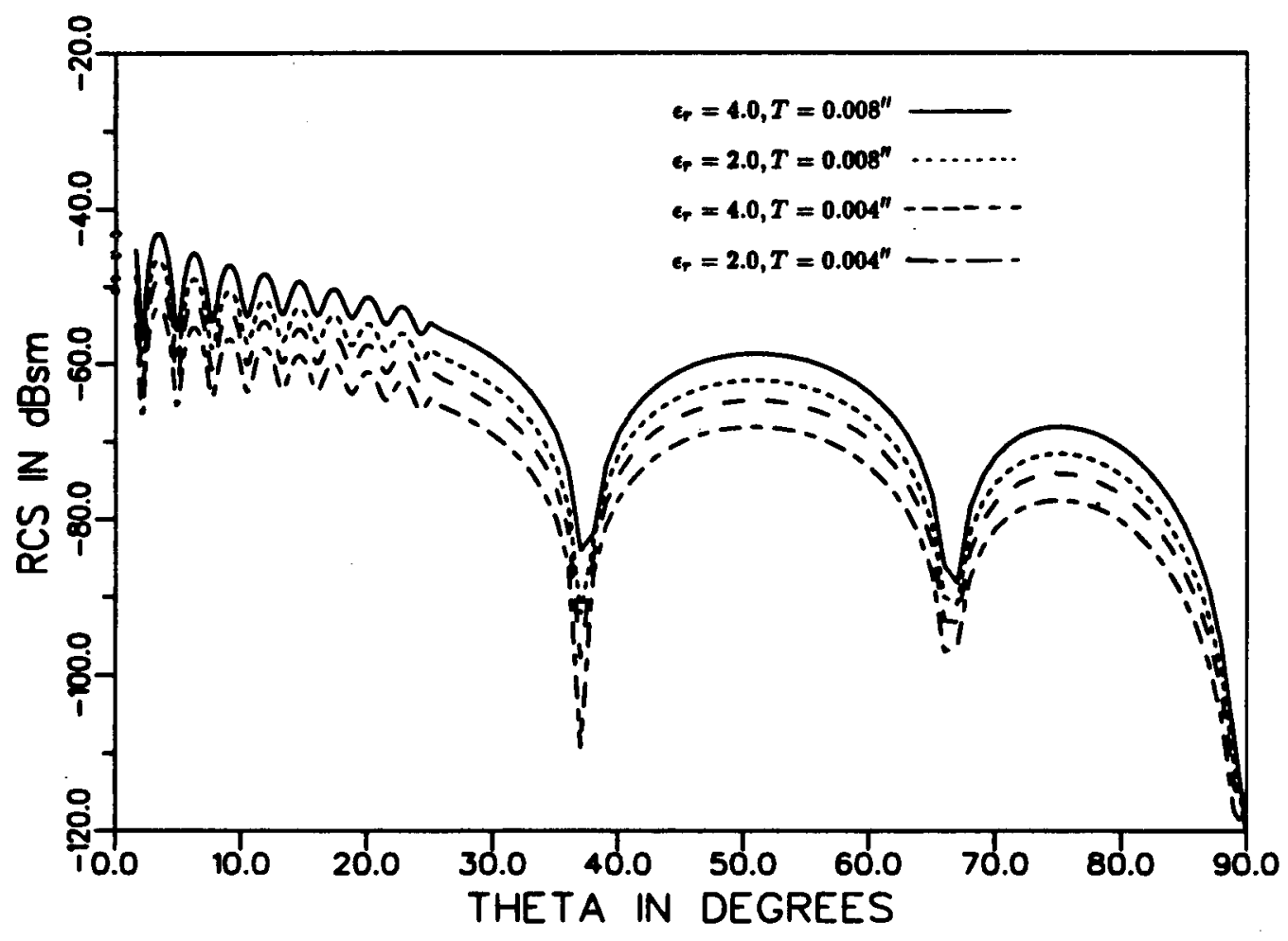

(c) Total RCS of the strap.

Figure 29: Continued. 


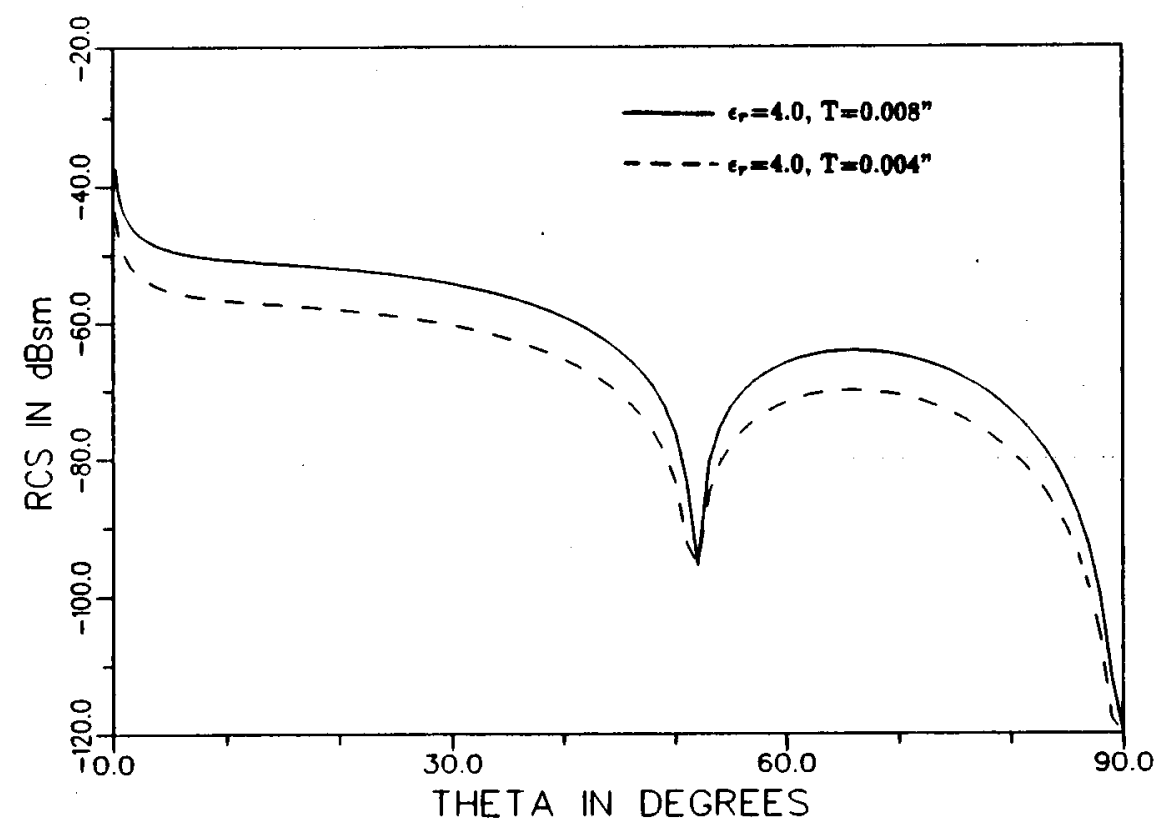

(a) RCS from the lit part of the strap.

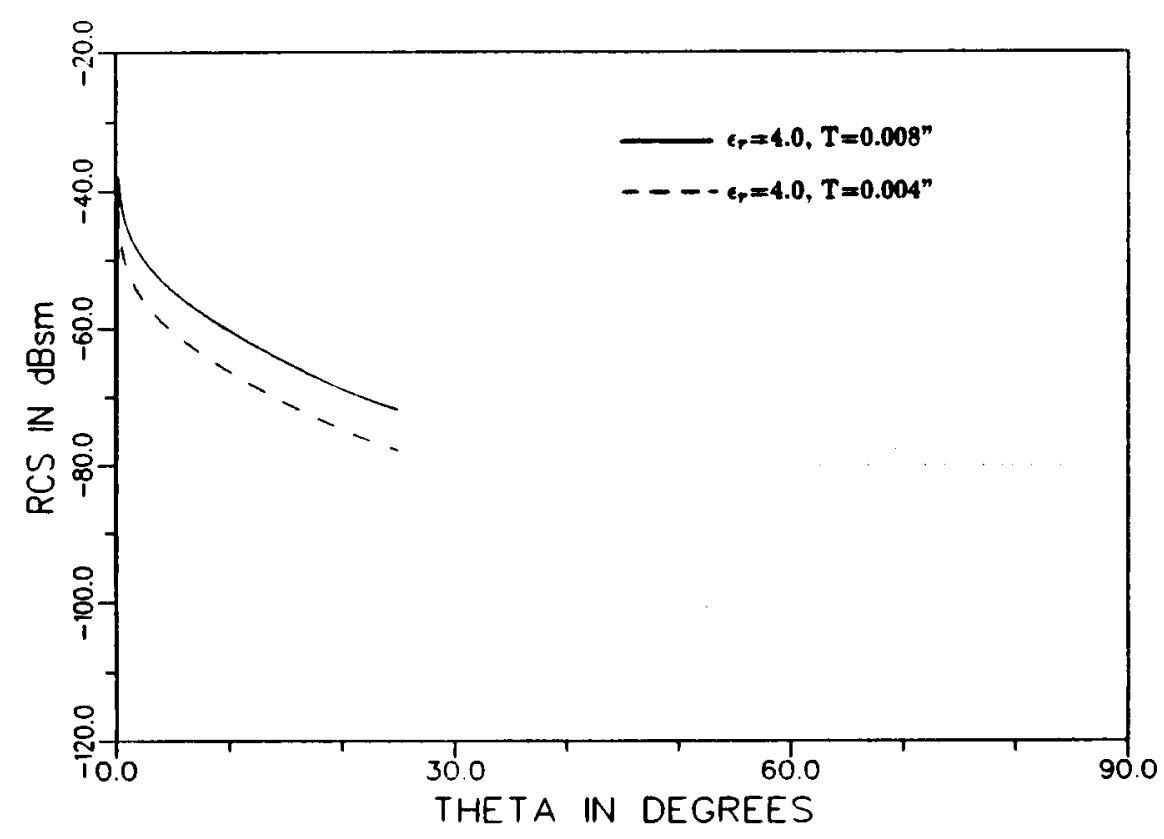

(b) RCS from the shadowed part of the strap.

Figure 30: RCS of a strap wrapped around an ogive with $a=14.76^{\prime \prime}, b=29.53^{\prime \prime}$, $\mathrm{L}=2.36^{\prime \prime}$ and $\mathrm{f}=4 \mathrm{GHz}$. 


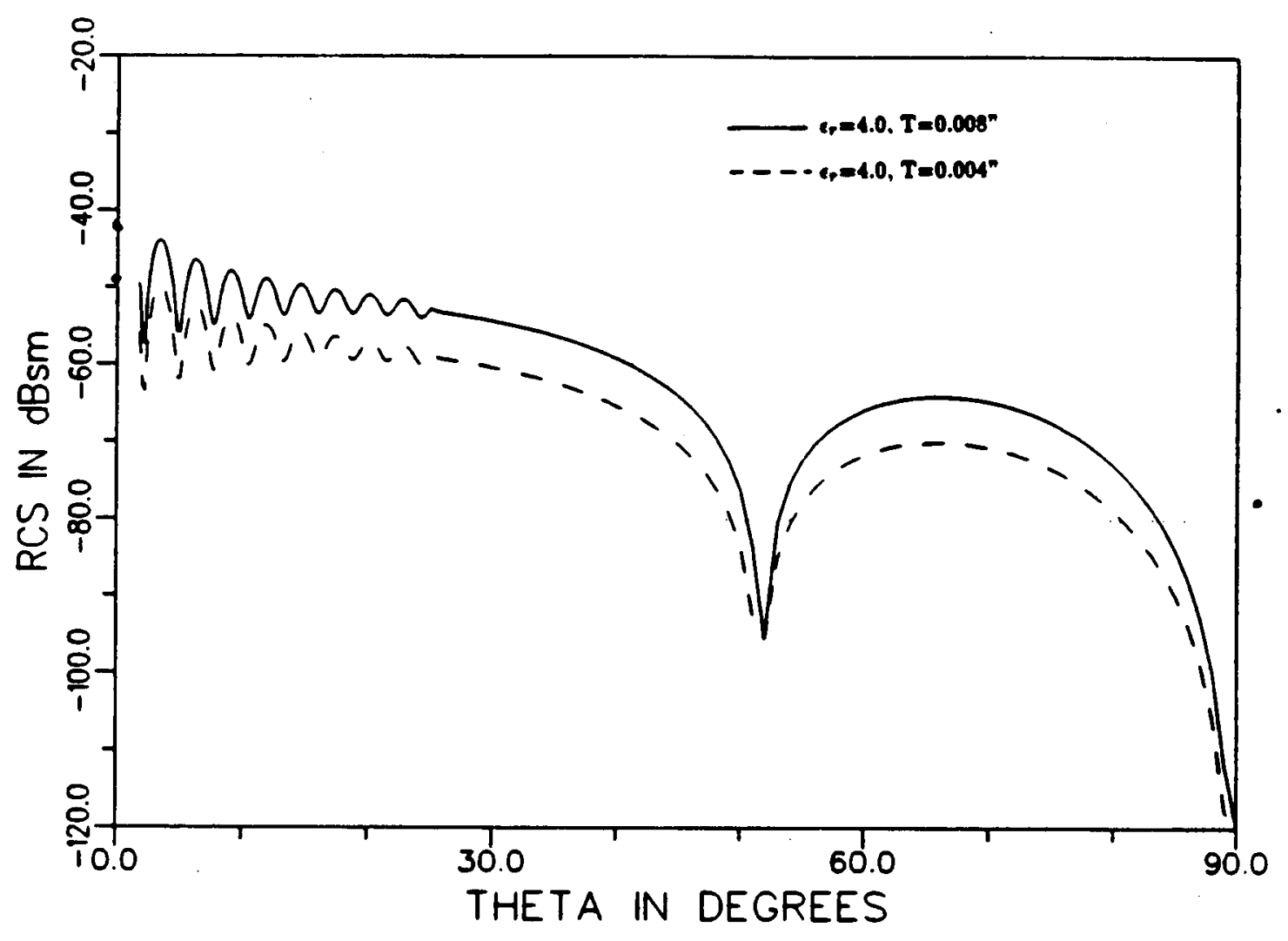

(c) Total RCS of the strap.

Figure 30: Continued. 


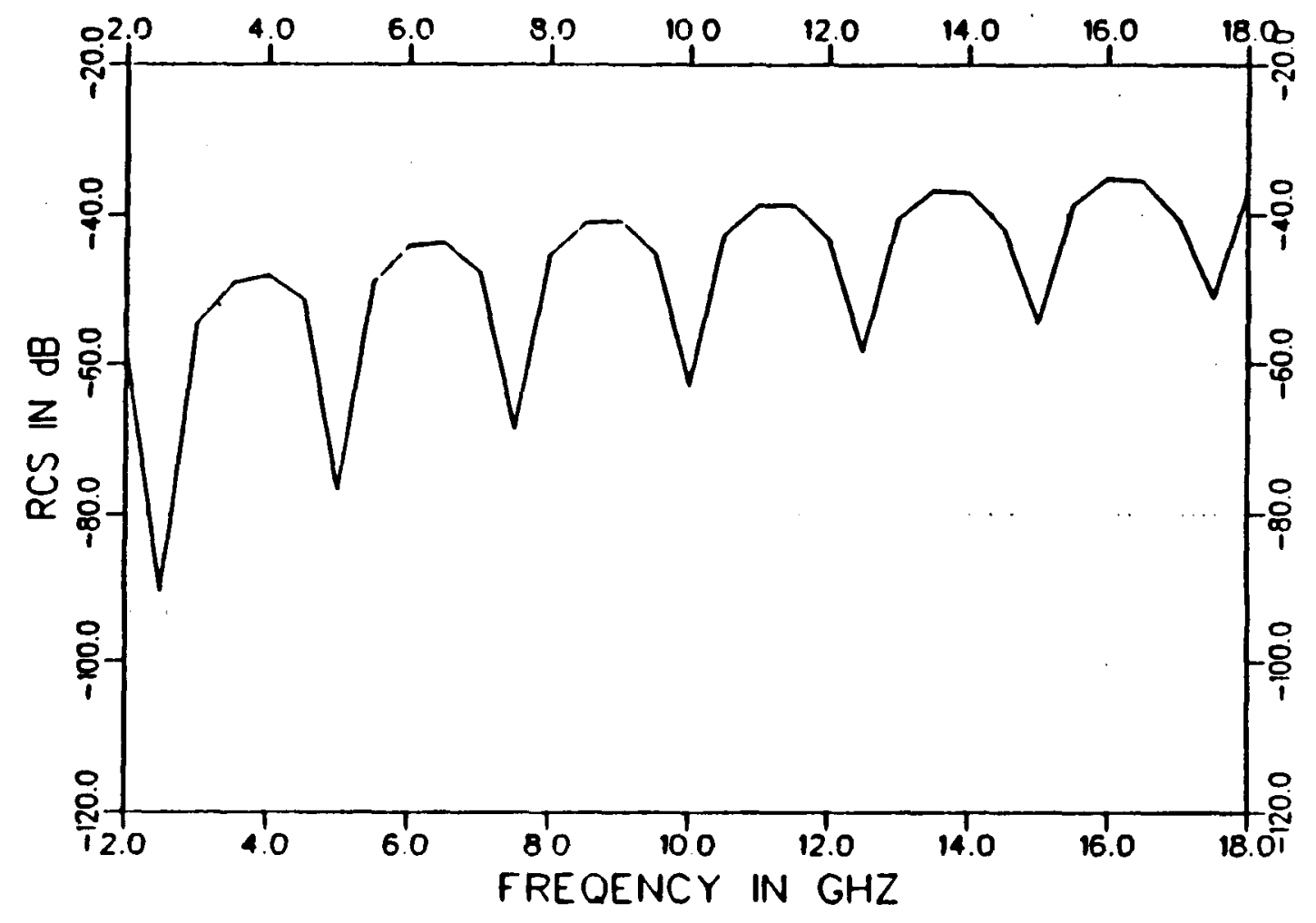

Figure 31: Nose-on RCS of the strap versus frequency with $a=14.76^{\prime \prime}, b=29.53^{\prime \prime}$, $\mathrm{L}=2.36^{\prime \prime}, \mathrm{T}=0.004^{\prime \prime}$ and $\epsilon_{r}=4.0$. 


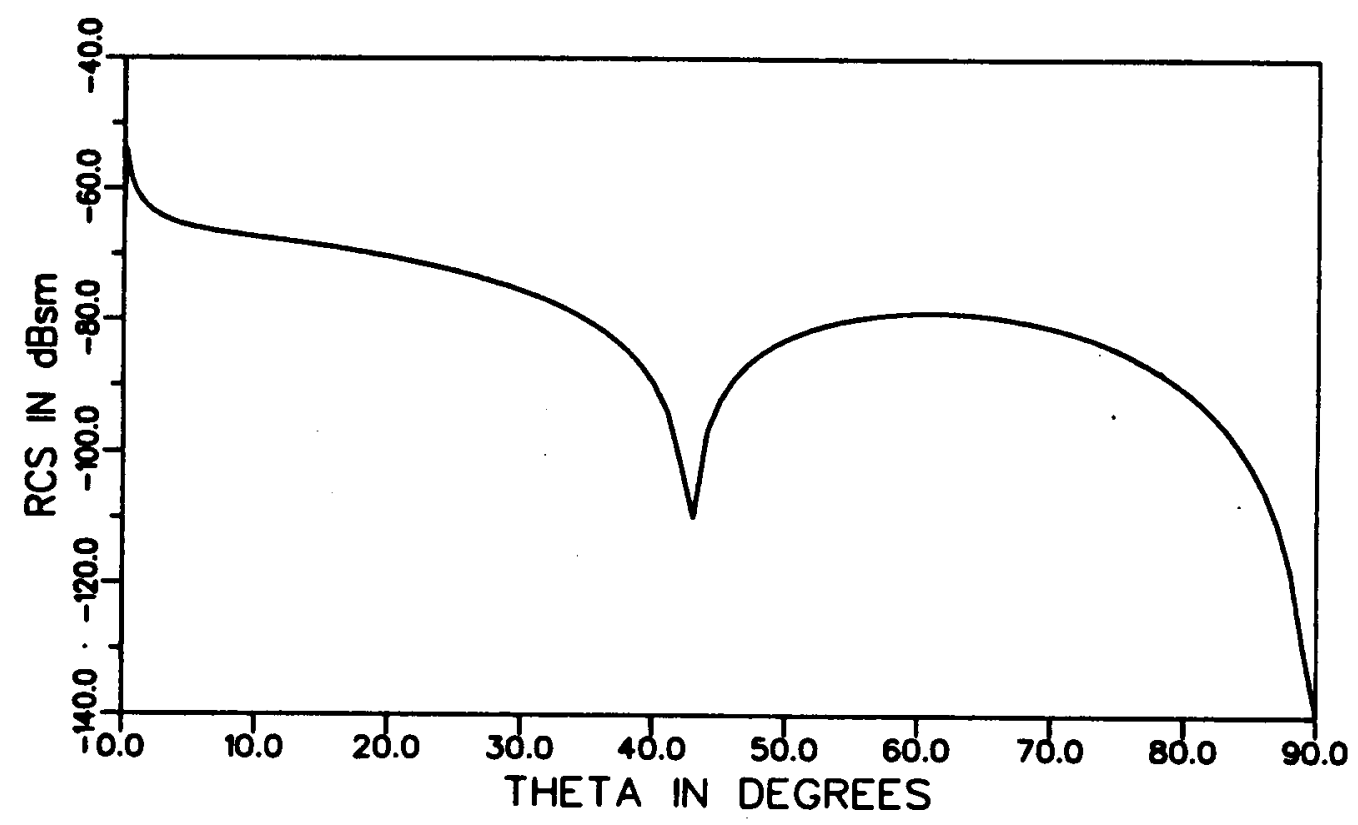

(a) RCS from the lit part of the strap.

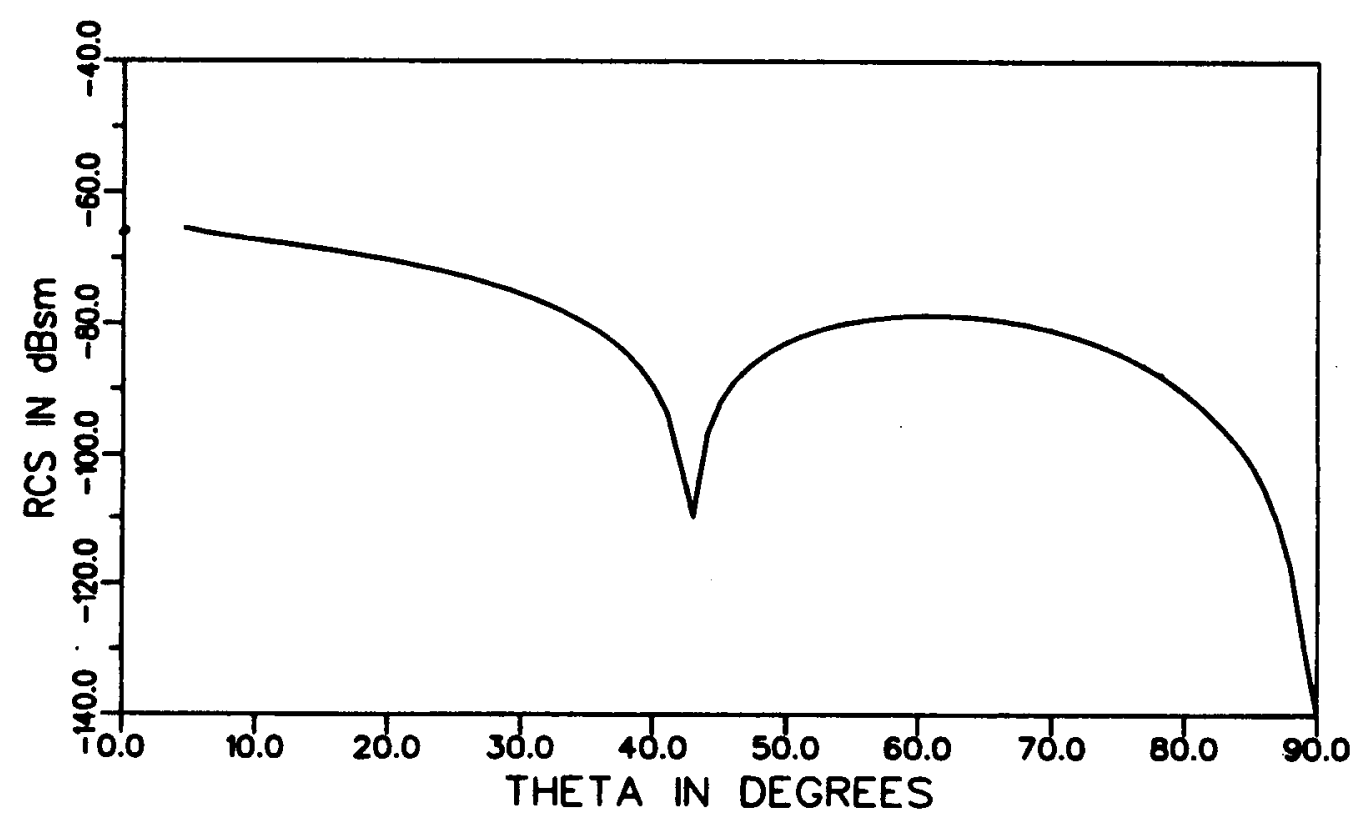

(b) Total RCS of the strap.

Figure 32: RCS of a strap wrapped around an ogive with $a=2.37^{\prime \prime}, b=18^{\prime \prime}$, $\mathrm{L}=2.0^{\prime \prime}, \mathrm{T}=0.004^{\prime \prime}, \epsilon_{r}=2.3$ and $\mathrm{f}=4 \mathrm{GHz}$. 


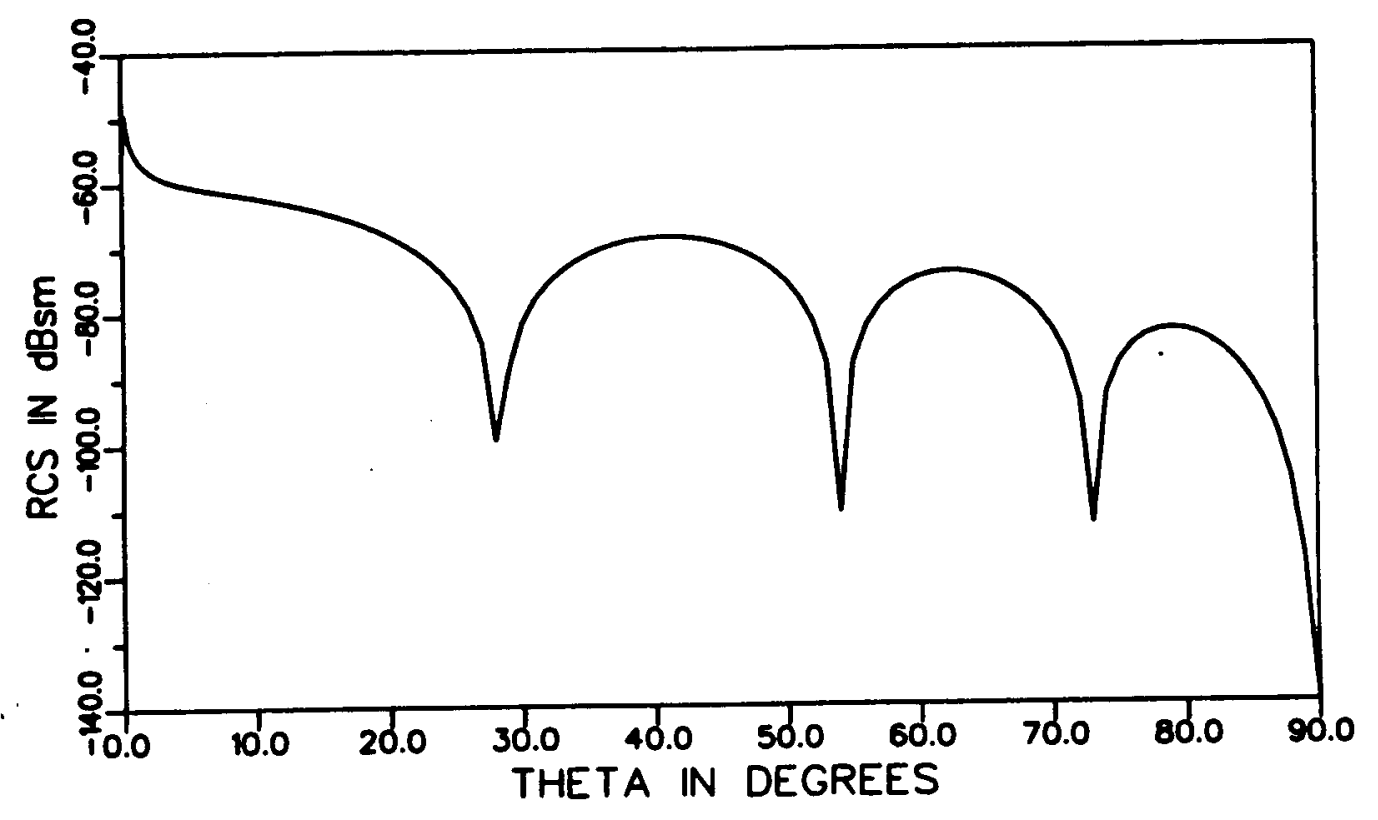

(a) RCS from the lit part of the strap.

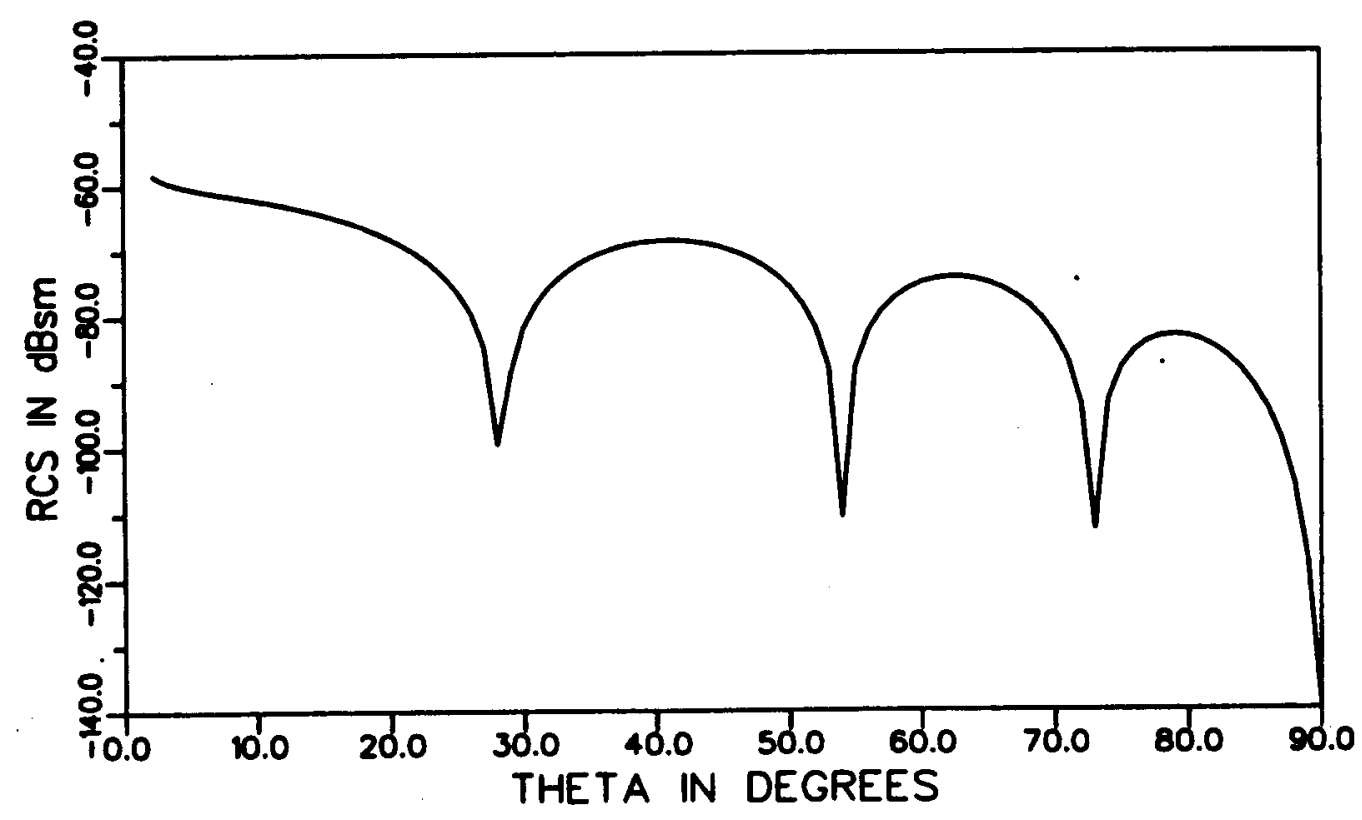

(b) Total RCS of the strap.

Figure 33: RCS of a strap wrapped around an ogive with $a=2.37^{\prime \prime}, b=18^{\prime \prime}$, $\mathrm{L}=2.0^{\prime \prime}, \mathrm{T}=0.004^{\prime \prime}, \epsilon_{r}=2.3$ and $\mathrm{f}=10 \mathrm{GHz}$. 
The creeping wave contribution to the RCS of the strap was not included because the cylinder perturbation method gives inaccurate results for such a thin ogive in that for a thin ogive the increase in the length of the creeping wave path with the increase in the observation angle, $\theta$ is larger than what the cylinder perturbation method can handle. Therefore for thin ogives, the cylinder perturbation method gives good results only for small $\theta$. But for small $\theta$, the solution for the shadowed part of the strap is not valid because of the caustic mentioned earlier. Another perturbation method such as the cone perturbation method [9] could be used. However, one will not gain too much from that, because the creeping wave term will stop at $\theta=15^{\circ}$ when it hits the tip of the ogive. Therefore, the RCS of the strap is computed at $\theta=0^{\circ}$ then the RCS of the lit part of the strap is computed alone beginning at $\theta=2$ or 3 degrees (i.e. outside the caustic region). One should not pay much attention to the discontinuity appearing in the RCS pattern, because one is interested in the RCS envelope rather than the exact RCS pattern. Figure 33 shows the RCS of the same strap at $10 \mathrm{GHz}$. The envelope of the RCS of the strap increased by about $8 \mathrm{~dB}$ with the increase of the frequency from 4 to 10 GHz. This can also be seen in the frequency domain plots as shown in Figure 15 .

\subsection{Empirical Design Formulas}

In this section, the computed results given in the previous section and the analytical expressions given in chapter III are used to establish some formulas which relates the RCS of the strap shown in Figure 34 to the different parameters of the problem. Such formulas are useful in designing and using the straps as a target support structure.

Lai and Burnside [2] provided a formula which relates the RCS of a strap in free space to its thickness, dielectric constant and the operating frequency. That 


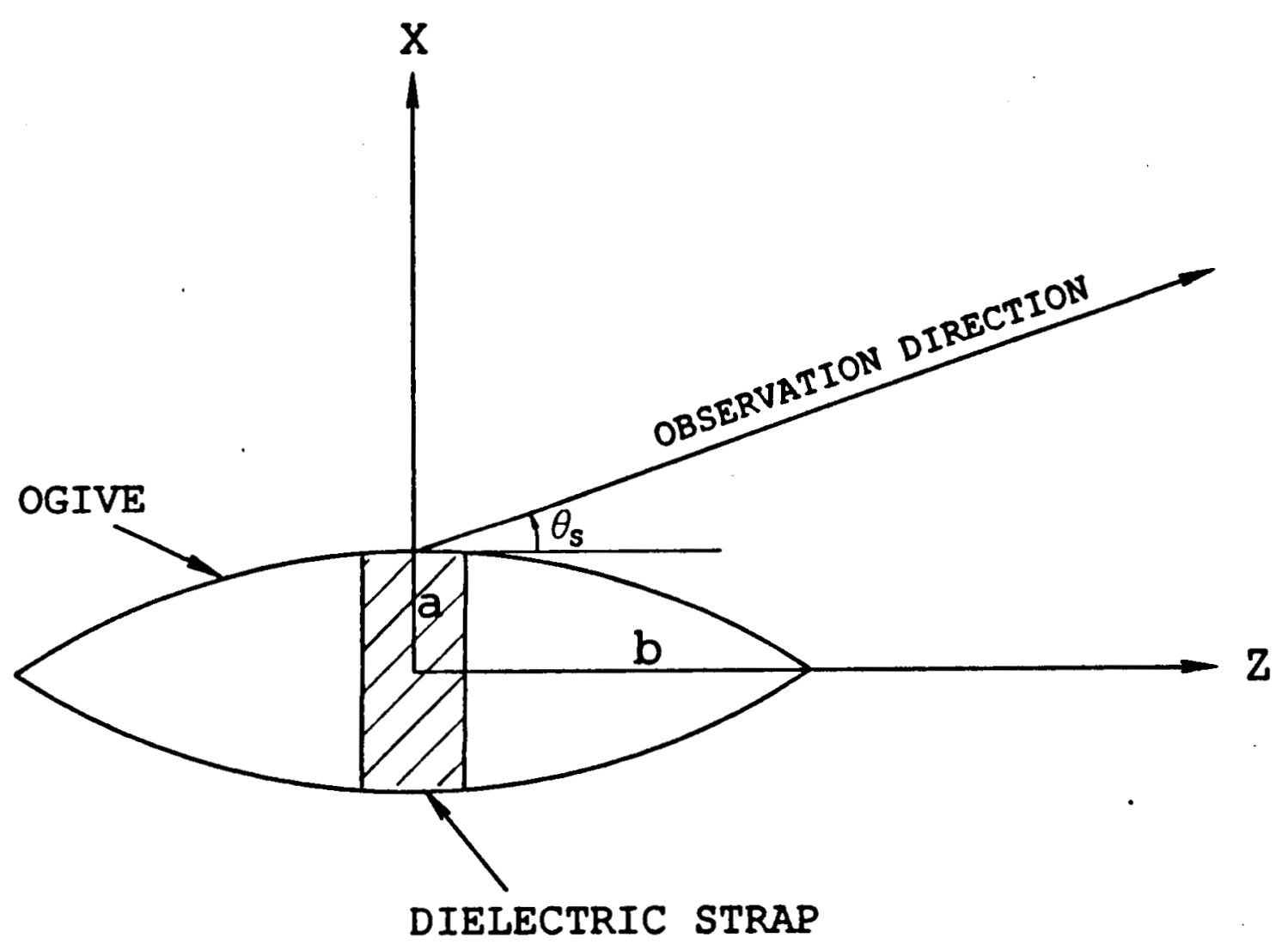

Figure 34: Strap wrapped around an ogive. 
formula stated that the RCS of the strap has $(T)^{1.7} \times\left(\epsilon_{r}\right)^{1.03}$ dependence. It can be seen that the results given in the previous section has the same dependence.

For $\theta_{s}=0$, Equation (3.39) along with the computed results of Section 4.1 are used to obtain the following equation:

$$
\begin{array}{r}
\sigma_{s}(0)=0.833 f-53.084+10 \log \left(\epsilon_{r}\right)^{1.03}+10 \log (T)^{1.7} \\
+20 \log \left(L_{s}\right)
\end{array}
$$

where

$\sigma_{s}(0)$ is the RCS of the strap in $\mathrm{dBsm}$ at $\theta_{s}=0$, and $\theta_{s}$ is the incidence angle as shown in Figure 34

$f$ is the frequency in $\mathrm{GHz}$

$T$ is the thickness in inches

$L_{s}$ is the strap length, for example $L_{s}=2 \pi a$ for the strap in Figure 34, and $\epsilon_{r}$ is the dielectric constant.

For $\theta_{s}$ outside the caustic region, say $\theta_{s} \geq 3^{\circ}$, Equation (3.21) along with the results of Section 4.1 are used to obtain that

$$
\begin{aligned}
& \sigma_{s}\left(\theta_{s}\right)=0.5 f-49+10 \log \left(\epsilon_{r}\right)^{1.03}+10 \log (T)^{1.7} \\
& +20 \log \left(\cos \left(\theta_{s}\right)\right)+10 \log \left(\frac{R_{1}}{2 \sin \left(\theta_{s}\right)}\right)+40 \log \left(H^{L}(\xi)\right)
\end{aligned}
$$

with

$$
\xi=\left(\frac{\pi f R_{2}\left(Q_{s}\right)}{11.8}\right)^{1 / 3} \sin (\theta s)
$$

where 
$Q s$ is the stationary point in the lit part of the strap, it is taken to be in the middle between the two edges of the strap

$R_{1}$ is the radius of curvature of the surface of the structure at $Q_{s}$ in a direction parallel to the edges of the strap

$R_{2}$ is the radius of curvature of the surface at $Q_{s}$ in a direction perpendicular to the edges of the strap.

$H^{L}(\xi)$ is the Fock radiation function, it is defined in chapter II, and

$\theta_{s}$ is the angle between the observation direction and the principal plane of the strap, as shown in Figures 34 through 36.

Note that the RCS envelope of the strap does not depend on the width of the strap; therefore, the width does not appear in Equations (4.2) and (4.3). Also, note that Equations (4.2) and (4.3) are good for general convex structures, and the ogive is chosen for demonstration purposes only.

Now, using Equations (4.2) and (4.3) the RCS envelope of the strap versus $f$, $\theta_{s}, T, \epsilon_{r}, R_{1}$, and $R_{2}$ may be found. Equation (4.2) is used to find the RCS at $\theta_{s}=0^{\circ}$, and Equation (4.3) is used to plot the RCS envelope for $\theta_{s}$ outside the caustic region, say $\theta_{s} \geq 3^{\circ}$. For $0^{\circ} \leq \theta_{s} \leq 3^{\circ}$, the RCS plot can be simply taken to be a constant value.

Figures 37 through 39 show the RCS envelope of the strap shown in Figure 34 with $a=2.37^{\prime \prime}$ and $b=18^{\prime \prime}$. Figure 37 shows the nose-on RCS envelope of the strap versus frequency with $T=0.002^{\prime \prime}, 0.004^{\prime \prime}$ and $0.006^{\prime \prime}$, and $\epsilon_{r}=2.3$. Figure 38 shows the RCS envelope with $\epsilon_{r}=2,3$ and 4 , and $T=0.004^{\prime \prime}$. Figure 39 shows the RCS envelope of the strap versus the observation angle, $\theta_{s}$ with $T=0.002$ " and $0.004^{\prime \prime}$, and $\epsilon_{r}=2.0$ and 4.0 . 


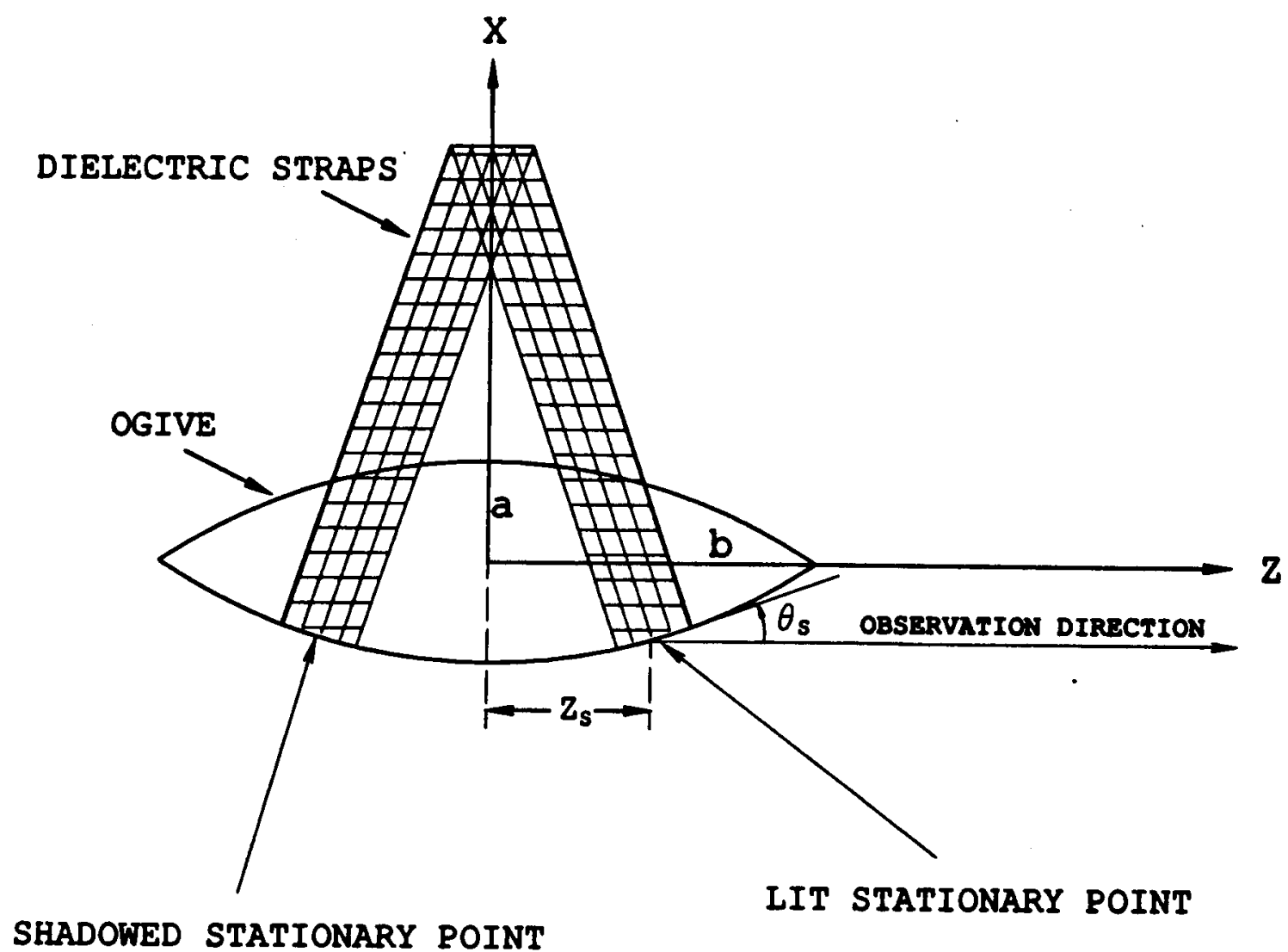

Figure 35: Ogive hung by straps. 


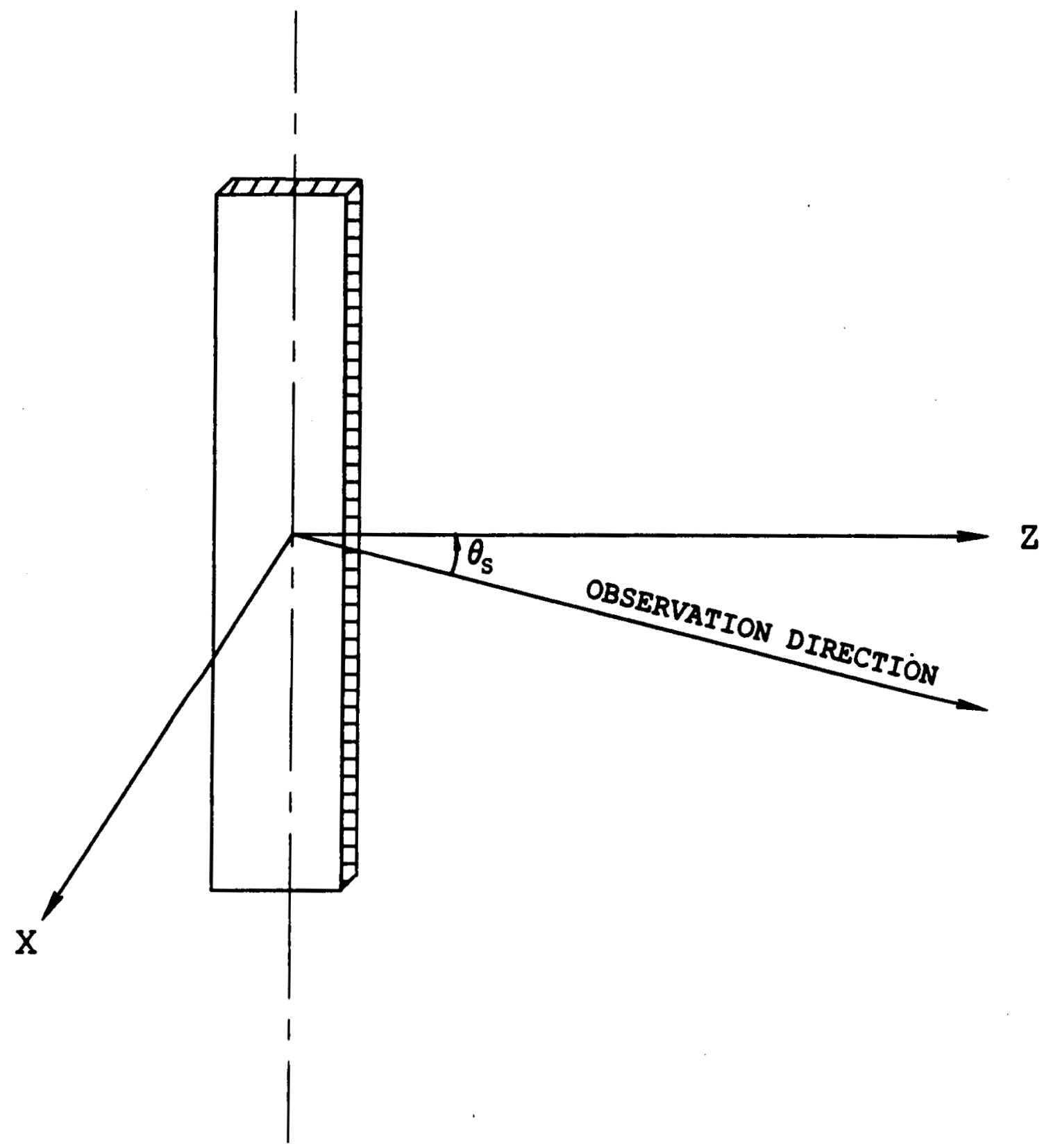

Figure 36: The angle $\theta_{s}$ with respect to the principle plane of the strap. 


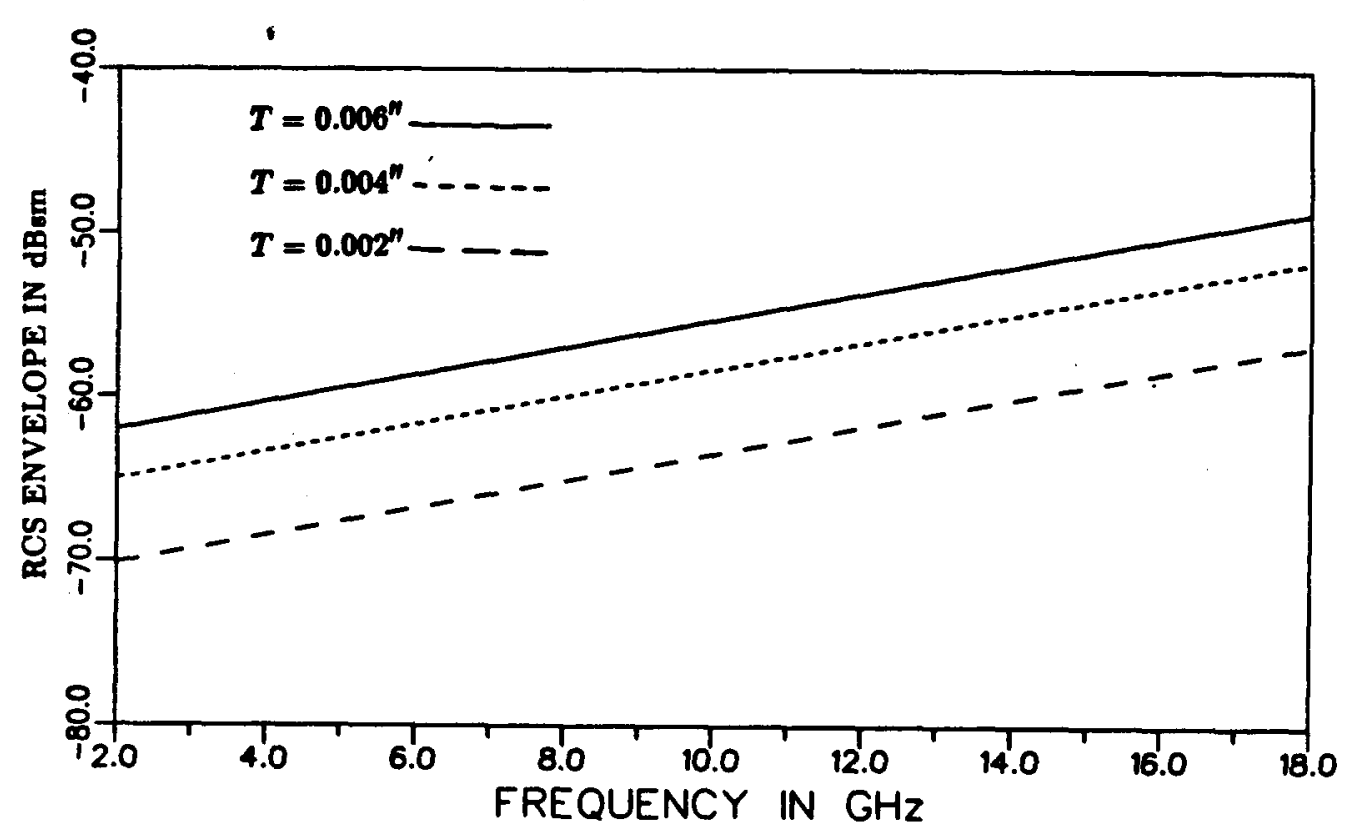

Figure 37: Nose-on RCS envelope of a strap wrapped around an ogive versus frequency with $a=2.37^{\prime \prime}, b=18^{\prime \prime}, \epsilon_{r}=2.3$ and $T=0.002^{\prime \prime}, 0.004^{\prime \prime}$ and $0.006^{\prime \prime}$.

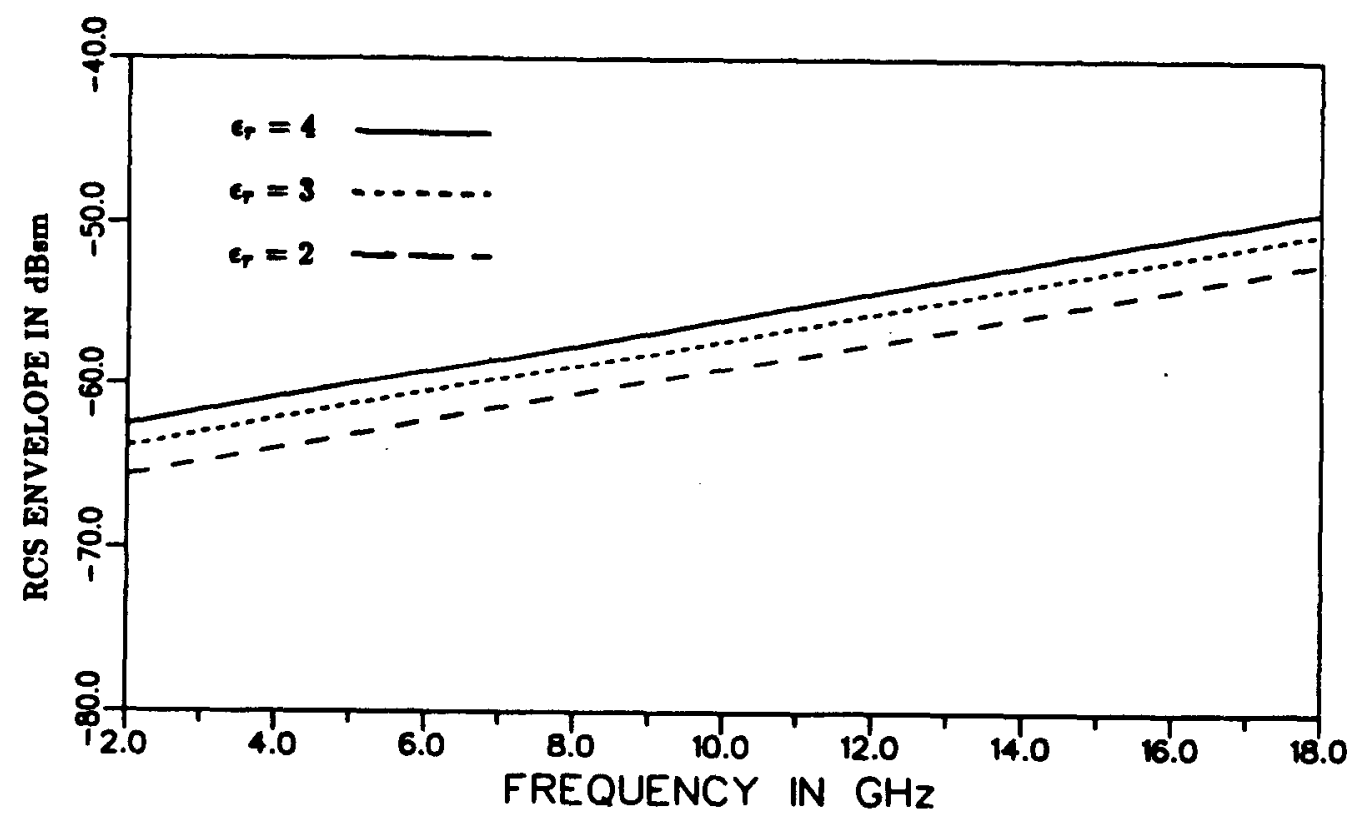

Figure 38: Nose-on RCS envelope of a strap wrapped around an ogive versus frequency with $a=2.37^{\prime \prime}, b=18^{\prime \prime}, T=0.004^{\prime \prime}$ and $\epsilon_{r}=2,3$ and 4. 


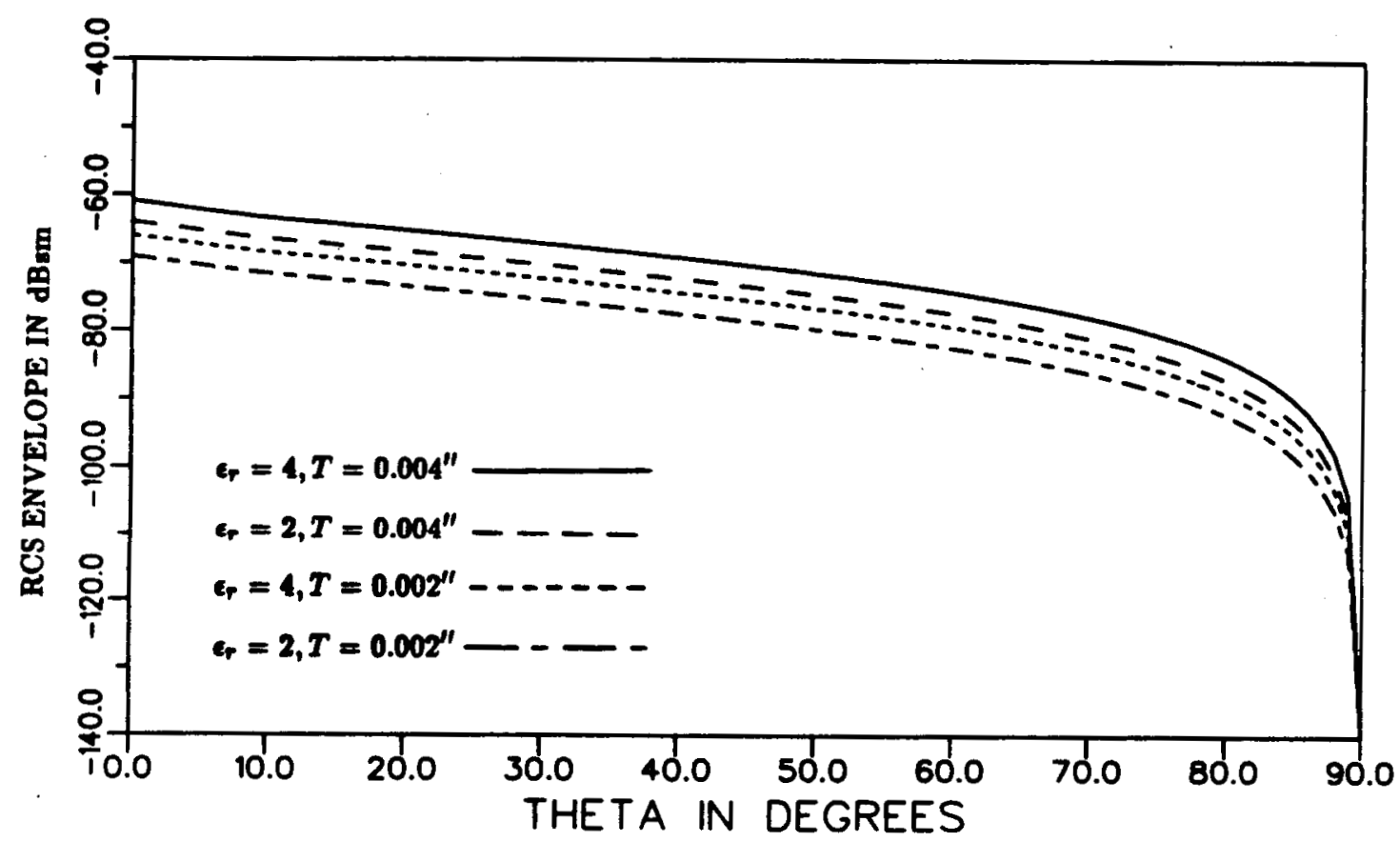

Figure 39: RCS envelope of a strap wrapped around an ogive with $a=2.37^{\prime \prime}$, $b=18^{\prime \prime}, T=0.002^{\prime \prime}, 0.004^{\prime \prime}$ and $\epsilon_{r}=2,4$ and $\mathrm{f}=4 \mathrm{GHz}$. 
Now, Equation (4.3) is used to estimate the the RCS envelope of the straps shown in Figure 35. It is seen that the straps are oriented in a way which minimizes its RCS. That orientation makes the backscattering field from the straps appear to be coming from two stationary points, these two stationary points are shown in Figure 35. One can see that the dominating contribution to the RCS of the straps is coming from the leading stationary point because it lies in the lit region; while, the other stationary point lies in the shadow region.

Figure 40 shows the RCS of the leading strap with $a=5^{\prime \prime}, b=20^{\prime \prime}$ and $Z_{s}=10^{\prime \prime}$, where $Z_{s}$ defines the stationary point location. Using the equations given in Section 3.2 , one finds that $R_{1}=3.8^{\prime \prime}, R_{2}=42.5^{\prime \prime}$ and $\theta_{s}=13.6^{\circ}$. The RCS of the leading strap is plotted for $T=0.002^{\prime \prime}$ and $0.004^{\prime \prime}$, and $\epsilon_{r}=2.0$ and 4.0. The contribution from the stationary point in the shadow region is not expected to have significant effect on the total RCS; therefore, the plot in Figure 40 is considered to be a reasonable first-order estimate for the RCS envelope of the straps shown in Figure 35.

The basic conclusions of this report are given in the next chapter. 


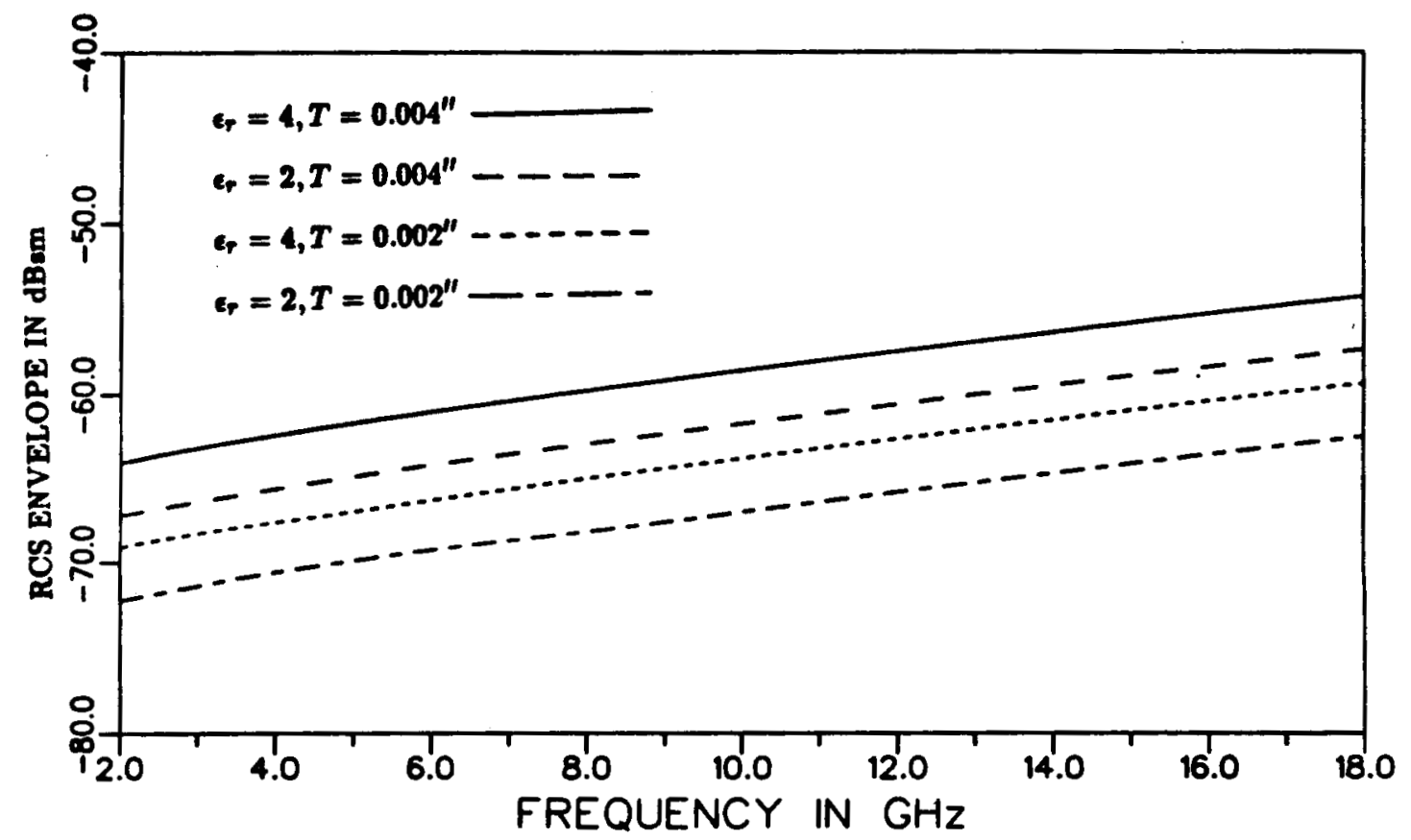

Figure 40: RCS envelope of the strap shown in Figure 35 versus frequency with $a=5^{\prime \prime}, b=20^{\prime \prime}, Z_{s}=10^{\prime \prime}, T=0.002^{\prime}, 0.004^{\prime \prime}$ and $\epsilon_{r}=2,4$. 


\section{CHAPTER V}

\section{SUMMARY AND CONCLUSIONS}

A method to calculate the electromagnetic scattered fields from a thin dielectric strap wrapped around a convex, perfectly conducting surface was presented. This was accomplished using a moment method technique to find the current excited within the strap by an incident plane wave. Then, Uniform Geometrical Theory of Diffraction, along with a stationary phase integration method was used to compute the fields scattered by the strap. This method was used to evaluate the RCS of a strap wrapped around a convex conducting structure. The computed RCS values showed reasonable agreement with measured results. It was shown that for low frequencies, say below $1 \mathrm{GHz}$, the strap scattering is very low. It increases with an increase in frequency, but it is still low at high frequencies. The RCS of the strap can be reduced by reducing the thickness or the dielectric constant of the strap. So a trade off can be made between the RCS of the strap and its physical properties. The H-plane RCS pattern is known to be much lower than the E-plane pattern; therefore, only the worst case, the E-plane pattern, was presented in this study.

Some empirical design formulas, which can help in designing the straps, are also given. These formulas are good for general convex structures and are expected to give resonable estimates for the RCS of the dielectric straps when used as a target support structure. 


\section{APPENDIX A \\ RAY DIVERGENCE FACTOR AND CAUSTIC DISTANCE}

The ray divergence factor $\sqrt{\frac{d \psi_{o}\left(Q^{\prime}\right)}{d \psi(Q)}}$ used in Equation (3.22) can be found as follows. First, we define the source location and the radiation direction. Next, we find the geodesic path that the ray will travel along it to reach the diffraction point, let the path length be $L$. At the diffraction point the radiation direction $\hat{t}$ must be tangent to the surface and aligned with the geodesic path as shown in Figure 41. The next step is to draw another geodesic path which has a length equal to $L$ and passes through the source location making an angle $d \gamma$ with the first geodesic path. The last step is to find the vector $\hat{t}^{a}$ which is tangent to the surface and aligned with the second geodesic path at its end. Then the ray divergence factor is given by

$$
\sqrt{\frac{d \psi_{o}\left(Q^{\prime}\right)}{d \psi(Q)}}=\sqrt{\frac{d \gamma}{\cos ^{-1}\left(\hat{t} \cdot \hat{t}_{a}\right)}}
$$

The above steps can be implemented numerically [10]. However, another approximate, but easier, method is followed here. This method is called the perturbation method [9]. The cylinder perturbation method is used here. It is good enough when the source exists near the region $Z=0$ on the ogive. The method uses some of the equations governing the geodesic path on a cylinder assuming that they are valid for the ogive.

The circular cylinder shown in Figure $42(\mathrm{a})$ is a developed surface; therefore, the geodesic path, $Q^{\prime} Q$, is a straight line on the unfolded planer surface. As shown 


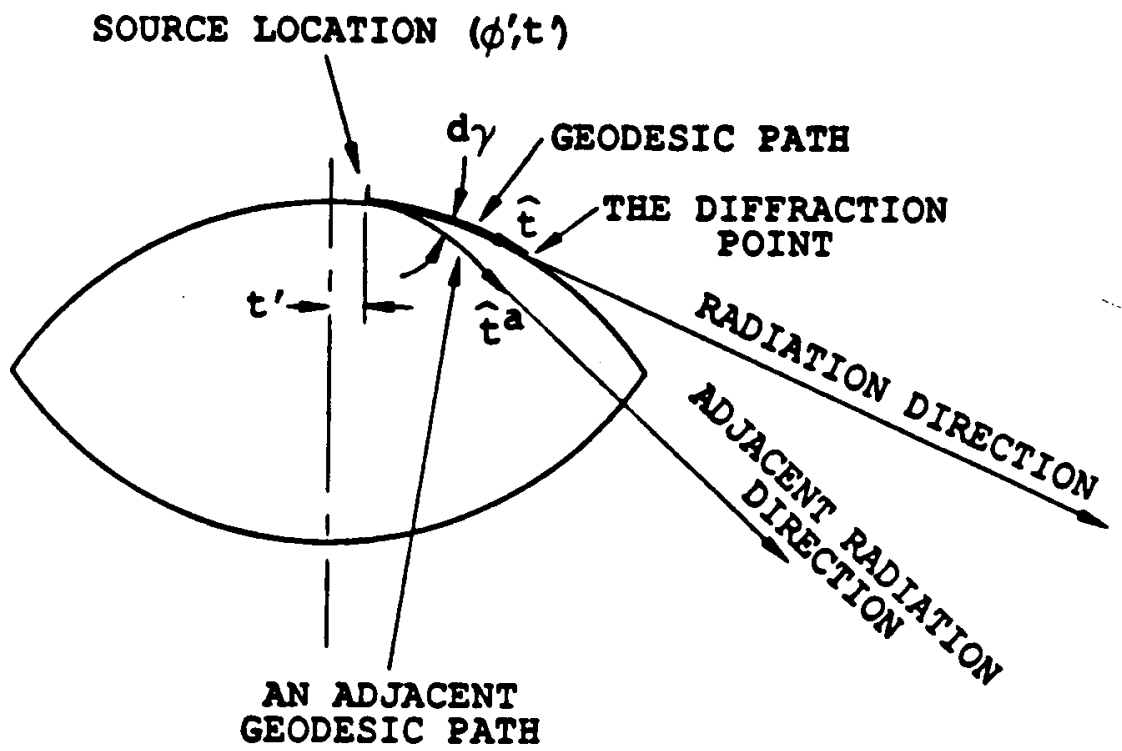

Figure 41: Creeping wave radiation from source over an ogive 


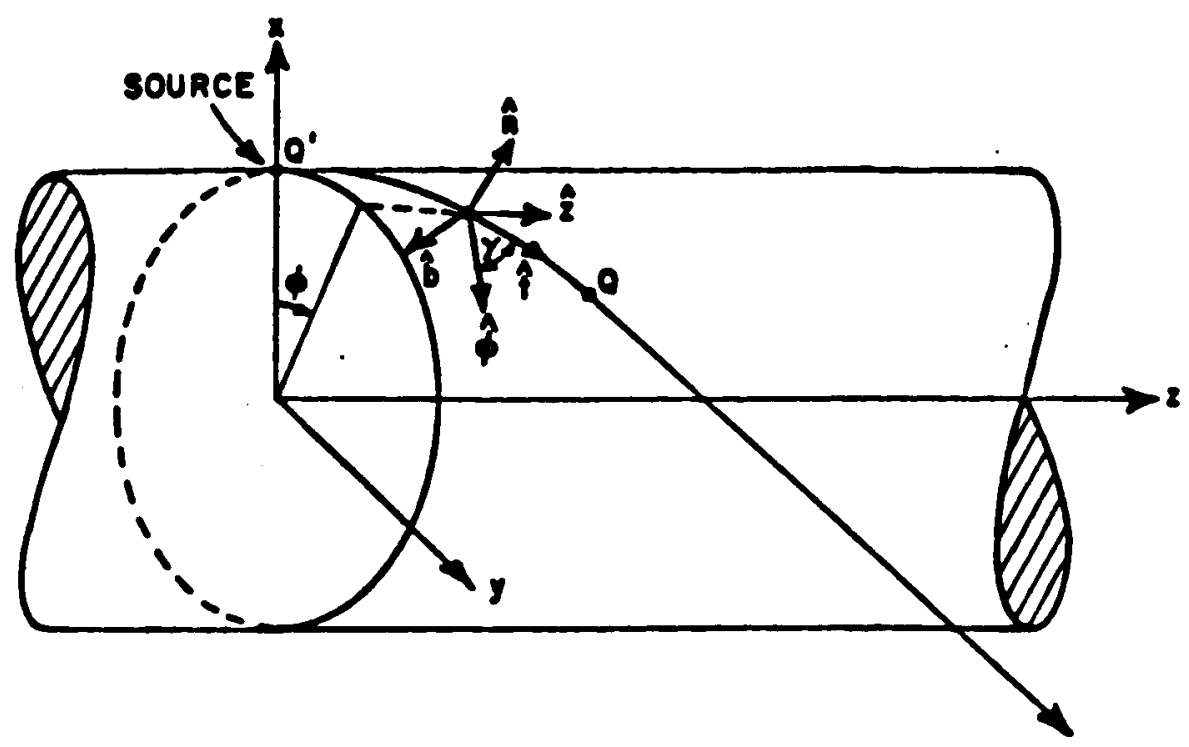

(a) True Cylinder

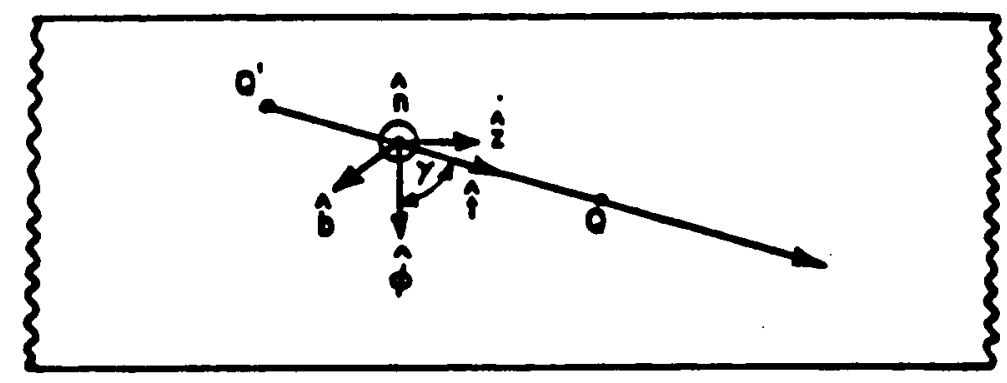

(b) Unfolded Planar Surface

Figure 42: Geodesic path on a developed cylinder. 


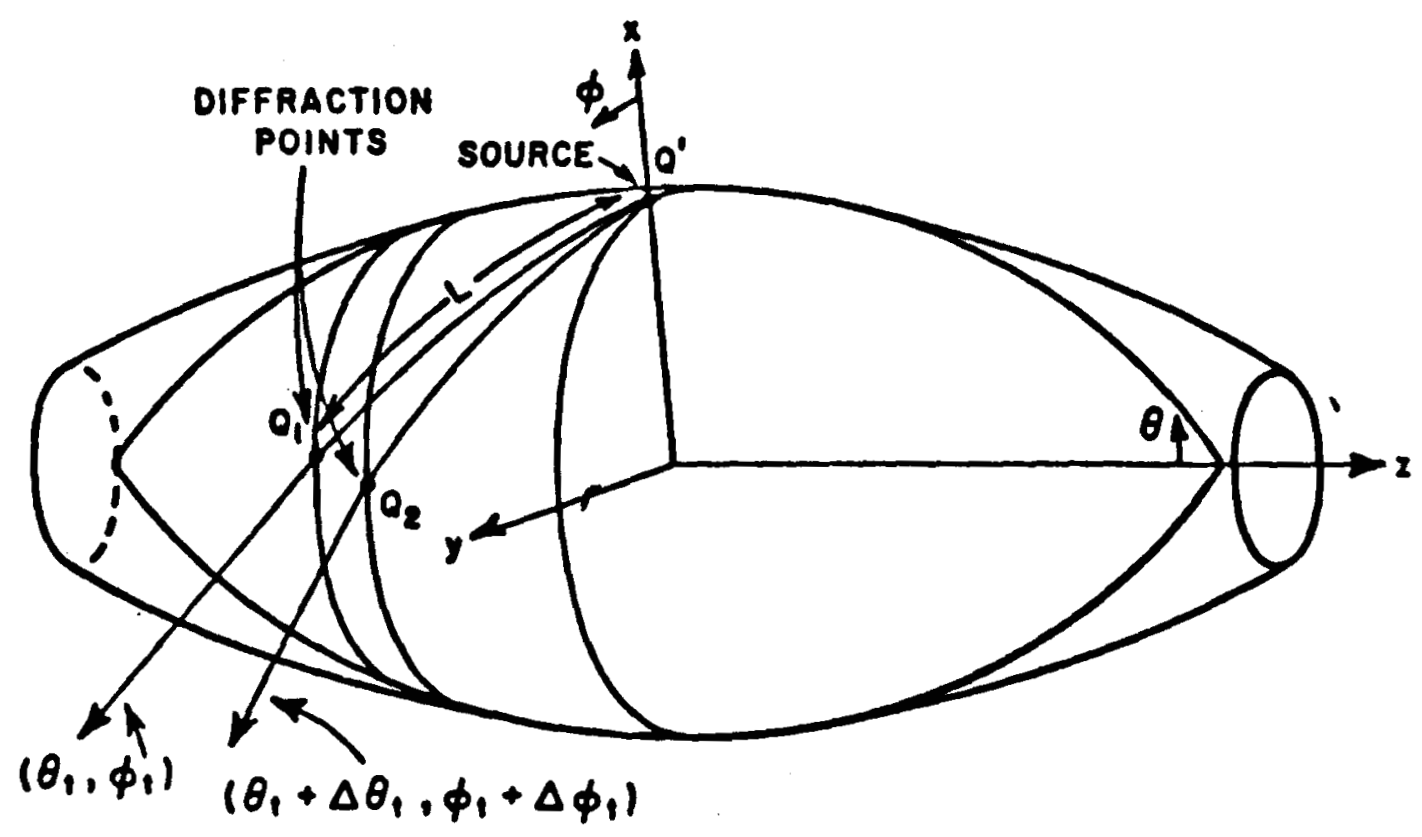

Figure 43: Circular cylinder perturbation.

in Figure $42(\mathrm{a})$ the geodesic unit tangent $\hat{t}$ is given by

$$
\hat{t}=\cos \gamma \hat{\phi}+\sin \gamma \hat{z}
$$

where

$$
\hat{\phi}=-\sin \phi \hat{x}+\cos \phi \hat{y}
$$

An important thing to put in mind is that, along a given geodesic path the angle $\gamma$, shown in Figure 42(b), is constant.

Now, as shown in Figure 43, the cylinder is perturbed by bending it around the ogive and the geodesics are to be resolved on this perturbed cylinder model. Then, using the fact that $\gamma$ is constant along a given geodesic path, $Q^{\prime} Q$, on the perturbed cylinder one obtains the geodesic equation

$$
\tan \gamma=\frac{s_{e}}{s_{r}}
$$




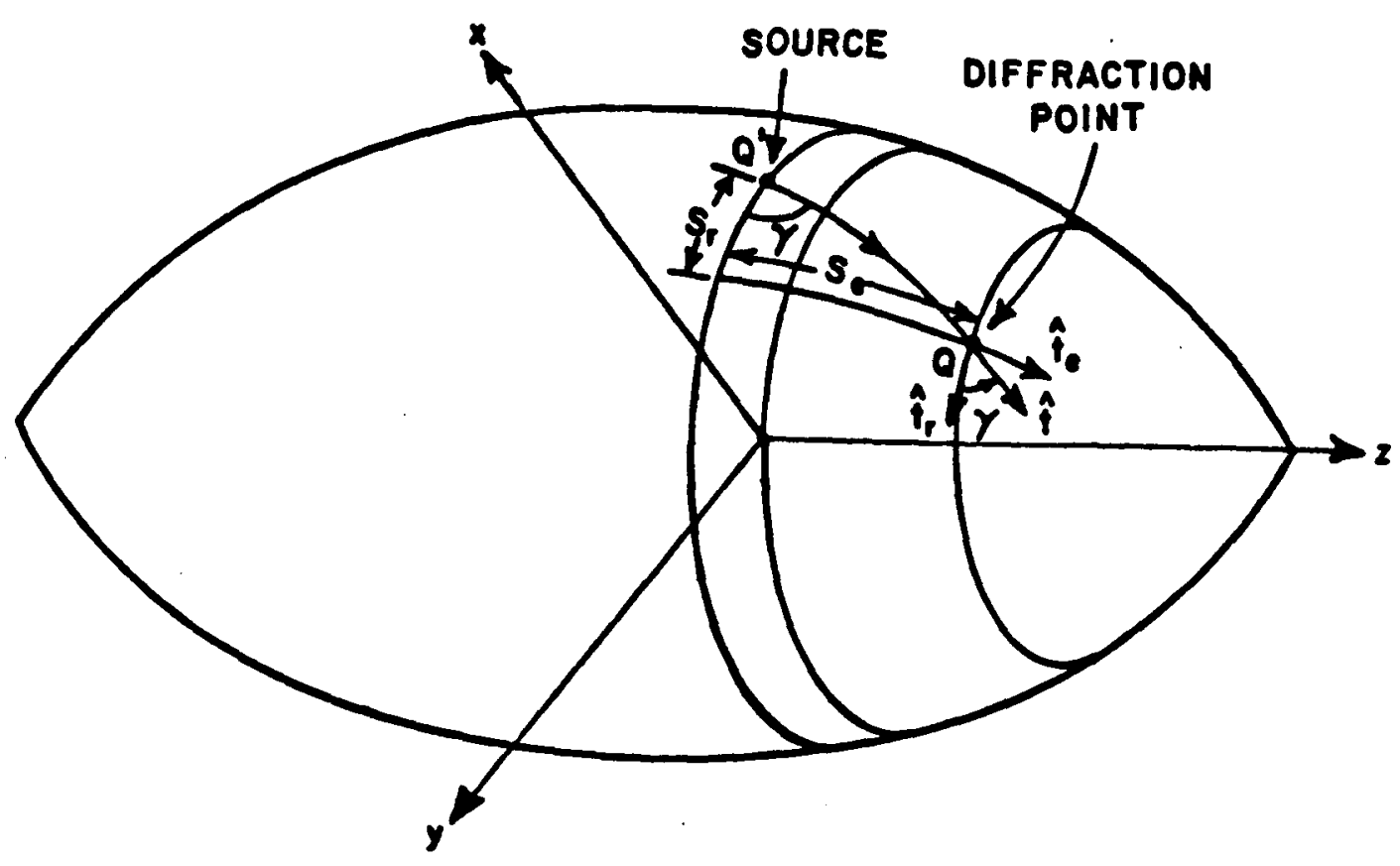

Figure 44: The different parameters for the geodesic path.

where $s_{e}$ and $s_{r}$ are as shown in Figure (A.4). And

$$
s_{r}=a \phi
$$

The point $Q$ is defined by $\nu$ and $\phi . \nu$ is equal to the observation angle $\theta$, and $\phi$ is taken to be zero. Now, since the cylinder is a developed surface

$$
s_{e}=\sqrt{L^{2}-s_{r}^{2}}
$$

Then

$$
\cot \gamma=\frac{a \phi}{\sqrt{L^{2}-a^{2} \phi^{2}}} .
$$

By differentiating both sides of the above equation with respect to $\phi$ and after simplification one gets

$$
-\csc ^{2}(\gamma) d \gamma=\frac{a L^{2}}{s_{e}^{3}} d \phi
$$


$d \phi$ can be found from the above expression for any $d \gamma$.

Now, one can simply see that

$$
s_{e}=r \nu-t^{\prime}
$$

let

$$
\nu^{\prime}=\frac{t^{\prime}}{r}
$$

then, one gets

$$
s_{e}=r\left(\nu-\nu^{\prime}\right)
$$

To find $d \nu$ due to a small $d \gamma$ we use Equation (A.4).

$$
\begin{gathered}
\tan \gamma=\frac{s_{e}}{s_{r}}=\frac{r\left(\nu-\nu^{\prime}\right)}{a \phi} \\
\tan (\gamma+d \gamma)=\frac{r\left(\nu+d \nu-\nu^{\prime}\right)}{a(\phi+d \phi)}, \text { and } \\
d \nu=\frac{a}{r} d \phi \tan (\gamma+d \gamma)-\nu+\nu^{\prime}
\end{gathered}
$$

Now, the adjacent diffraction point $Q^{a}:(\phi+d \phi, \nu+d \nu)$ is known and

$$
\begin{gathered}
\hat{t}^{a}=\hat{t}_{r}^{a} \cos (\gamma+d \gamma)+\hat{t}_{e}^{a} \sin (\gamma+d \gamma) \\
\hat{t}=\hat{t}_{r} \cos (\gamma)+\hat{t}_{e} \sin (\gamma)
\end{gathered}
$$

where $\hat{t}$ and $\hat{t}_{e}$ are functions of the point location, they are given in Section 3.2. The ray divergence factor is then given by

$$
\sqrt{\frac{d \psi_{o}\left(Q^{\prime}\right)}{d \psi(Q)}}=\sqrt{\frac{d \gamma}{\cos ^{-1}(\hat{t} \cdot \hat{t} a)}} .
$$

where the angle $d \gamma$ can be conveniently chosen as $1^{o}$.

The caustic distance $\rho_{2}^{d}$ is easily found. It is the distance between the diffraction point $(\phi, \nu)$ on the ogive and the intersection point of $\hat{t}$ and $\hat{t}^{a}$ when they pass through $(\phi, \nu)$ and $(\phi+d \phi, \nu+d \nu)$, respectively. 


\section{LIST OF REFERENCES}

[1] Kin-Yue Albert Lai and W.D. Burnside, "A GTD Analysis of Ogive Pedestal," Technical Report 716148-8, The Ohio State University, ElectroScience Laboratory, prepared under Grant No. NSG-1013, for NASA Langley Research Center, Hampton, VA, March 1986.

[2] Albert Lai and W.D. Burnside, "Optimal Mounting Methods in Compact Range Application," Technical Report No. 719267-1, The Ohio State University ElectroScience Laboratory, prepared under Grant GU-0160 for Boeing Aerospace Company, Seattle, Washington, February 1987.

[3] Inder J. Gupta, Albert K. Lai, Walter D. Burnside, 'Scattering by Dielectric Straps with Potential Application as Target Support Structures," submitted for publication to IEEE Transactions on Antennas and Propagation.

[4] J.H. Richmond, "Scattering by a Dielectric Cylinder of Arbitrary CrossSection Shape," IEEE Transactions on Antennas and Propagation, Vol. AP13, No. 3, pp. 334-341, May 1965.

[5] R.G. Rojas "Scattering by an Inhomogeneous Dielectric/Ferrite Cylinder of Arbitrary Cross-Section Shape - Oblique incidence Case," IEEE Transactions on Antennas and Propagation, Vol. AP-36, No. 2, pp. 238-246, February 1988.

[6] Prabhakar H. Pathak, Nan Wang, Walter D. Burnside and Robert G. Kouyoumjian, "A Uniform GTD Solution for the Radiation from Sources on a Convex Surface," IEEE Transactions on Antennas and Propagation, Vol. AP-29, No. 4, pp. 609-622, July 1981 .

[7] P.H. Pathak, "Uniform GTD Solutions for a Class of Problems Associated with the Diffraction by Smooth Convex Surfaces," Short Course on the Modern Geometrical Theory of Diffraction, Vol. 1, The Ohio State University, Department of Electrical Engineering, Columbus, Ohio.

[8] D.S. Jones, and M. Kline, "Asymptotic Expansion of Multiple Integrals and the Method of Stationary Phase," J. Math. Phys., 37, pp. 1-28, 1958.

[9] J.J. Kim, W.D. Burnside, "Simulation and Analysis of Airborne Antenna Radiation Patterns," Technical Report $716199-1$, The Ohio State University 
ElectroScience Laboratory, prepared under Grant NSG-1498 for NASA Langley Research Center, Hampton, VA, December 1984.

[10] W.D. Burnside, "Analysis of On-Aircraft Antenna Patterns," Technical Report 3390-1, The Ohio State University ElectroScience Laboratory, prepared under Grant N62269-72-0354 for Naval Air Development Center, Warminster, PA, August 1972. 